Prepared in cooperation with the City of Cedar Rapids, lowa

\title{
Hydrologic and Water-Quality Conditions in the Cedar River Alluvial Aquifer, Linn County, lowa, 1990-2019
}

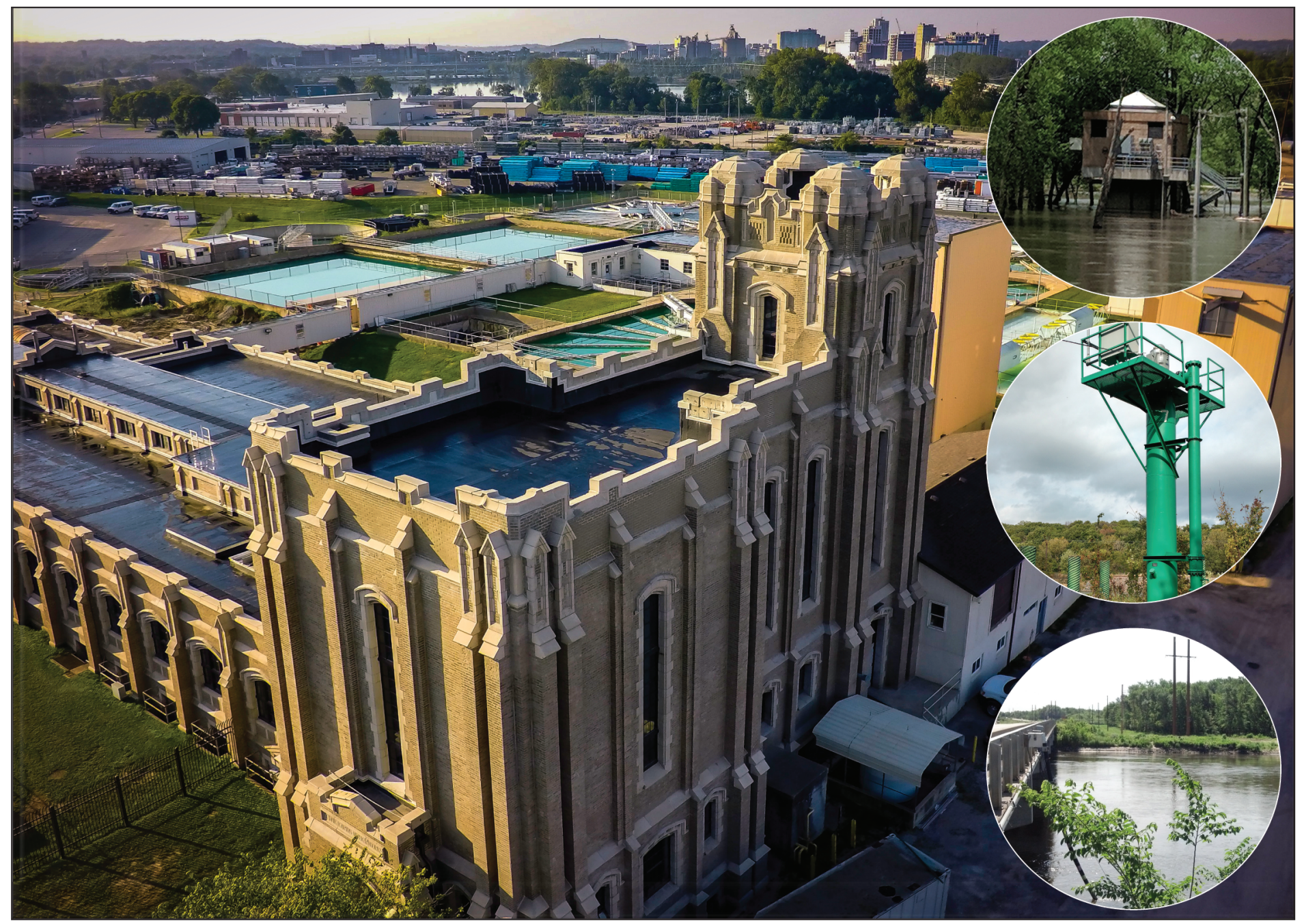

Scientific Investigations Report 2021-5110 


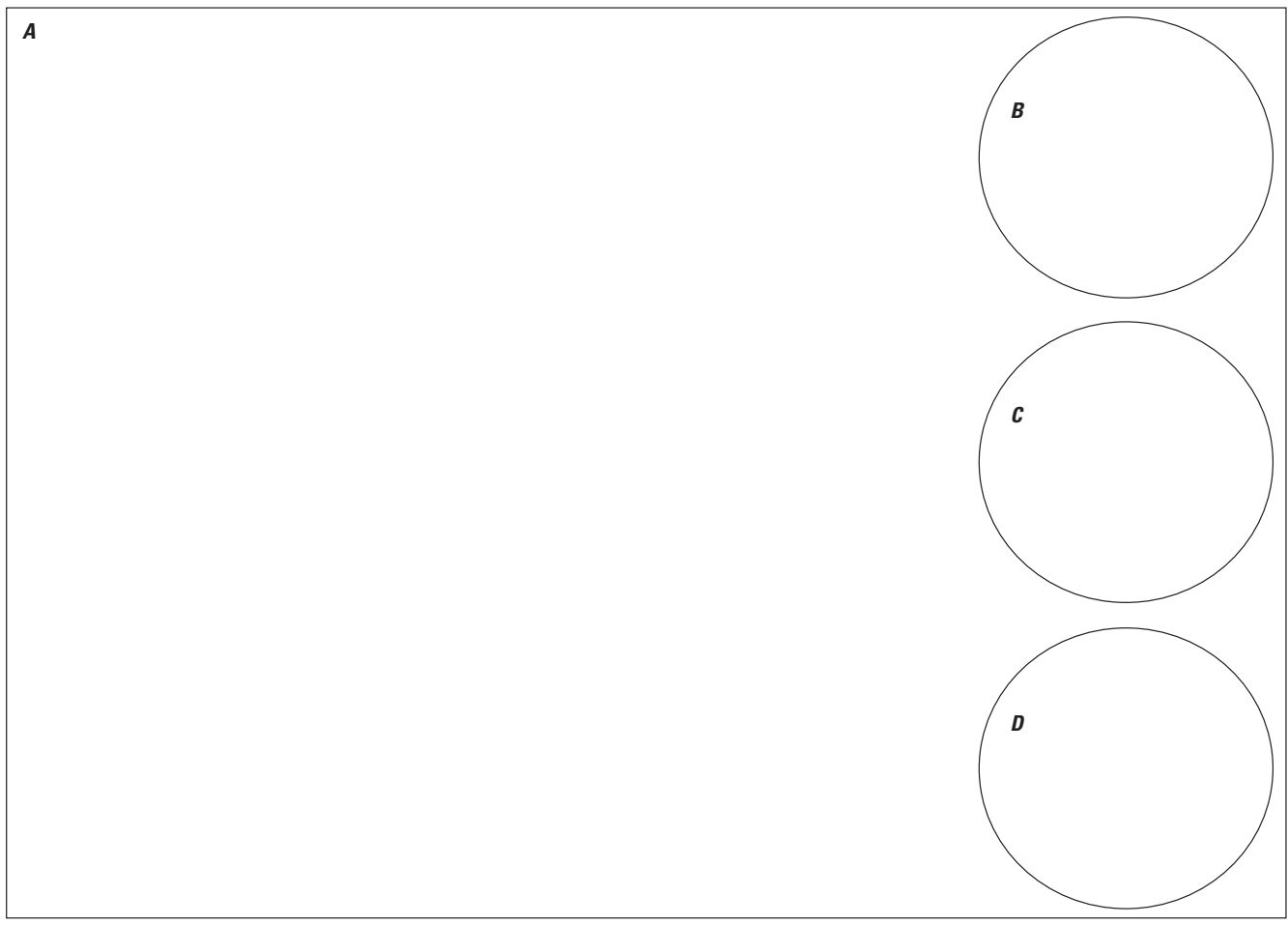

Cover: A. Photograph showing a water treatment plant in Cedar Rapids, lowa, on August 15, 2015. Photograph by City of Cedar Rapids, used with permission. B. Photograph showing a Cedar River alluvial aquifer production well in Cedar Rapids, lowa, on October 3, 2017. Photograph by Jason McVay, U.S. Geological Survey (USGS). C. Photograph showing a flooded Ranney collector well in Cedar Rapids, lowa, on May 9, 2018. Photograph by Shannon Meppelink, USGS. D. Photograph showing a water-quality monitoring site on the Cedar River at Blairs Ferry Road upstream from Cedar Rapids, lowa, on June 15, 2017. Photograph by S.J. Kalkhoff, USGS. 


\section{Hydrologic and Water-Quality Conditions in the Cedar River Alluvial Aquifer, Linn County, lowa, 1990-2019}

By Stephen J. Kalkhoff

Prepared in cooperation with the City of Cedar Rapids, lowa

Scientific Investigations Report 2021-5110 


\section{U.S. Geological Survey, Reston, Virginia: 2021}

For more information on the USGS - the Federal source for science about the Earth, its natural and living resources, natural hazards, and the environment-visit https://www.usgs.gov or call 1-888-ASK-USGS.

For an overview of USGS information products, including maps, imagery, and publications, visit https://store.usgs.gov/.

Any use of trade, firm, or product names is for descriptive purposes only and does not imply endorsement by the U.S. Government.

Although this information product, for the most part, is in the public domain, it also may contain copyrighted materials as noted in the text. Permission to reproduce copyrighted items must be secured from the copyright owner.

Suggested citation:

Kalkhoff, S.J., 2021, Hydrologic and water-quality conditions in the Cedar River alluvial aquifer, Linn County, lowa, 1990-2019: U.S. Geological Survey Scientific Investigations Report 2021-5110, 61 p., https://doi.org/10.3133/ sir20215110.

Associated data for this publication:

Meppelink, S.M., and Kalkhoff, S.J., 2021, Hydrologic and water quality data from the Cedar River and Cedar River alluvial aquifer, Linn County, lowa, 1990-2019: U.S. Geological Survey data release, https://doi.org/10.5066/ P9Z7VKOU.

U.S. Geological Survey, 2020, USGS water data for the Nation: U.S. Geological Survey National Water Information System database, https://doi.org/10.5066/F7P55KJN.

ISSN 2328-0328 (online) 


\section{Acknowledgments}

The authors would like to acknowledge the City of Cedar Rapids, lowa, Utilities Water Division, which provided a substantial amount of the funding for this project. Water-quality samples also were collected, and streamflow was measured by personnel from the U.S. Geological Survey office in lowa City, lowa. Technical and editorial reviews of initial drafts by Jennifer Murphy, Lance Gruhn, and Rebekah Davis of the U.S. Geological Survey provided invaluable comments and suggestions that greatly improved this report. 



\section{Contents}

Acknowledgments ……...................................................................................................................

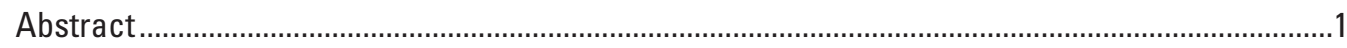

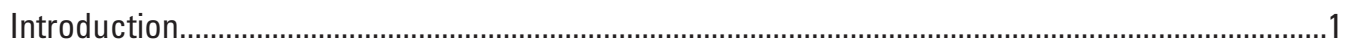

Description of the Problem ...............................................................................................

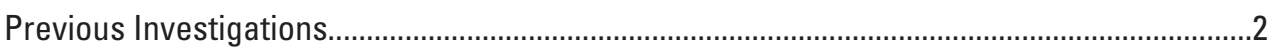

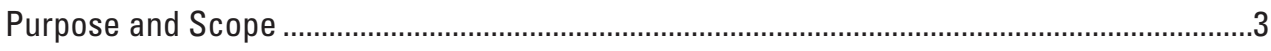

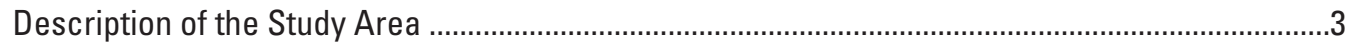

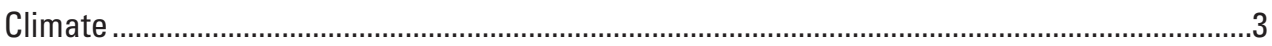

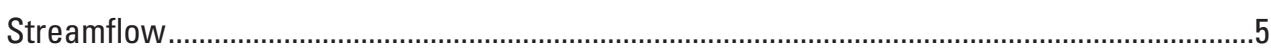

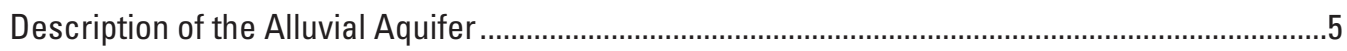

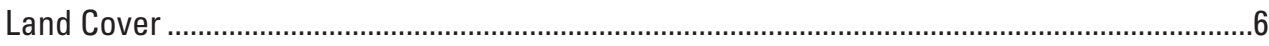

Geology

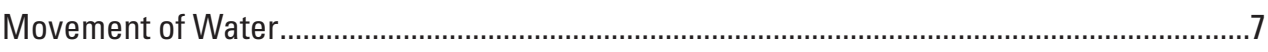

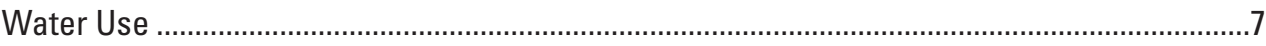

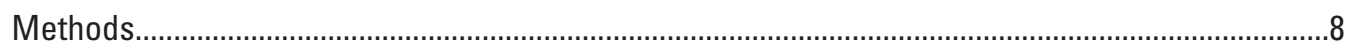

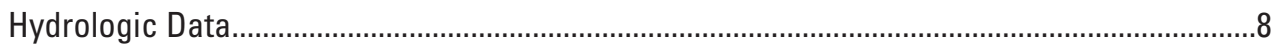

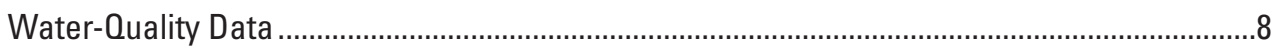

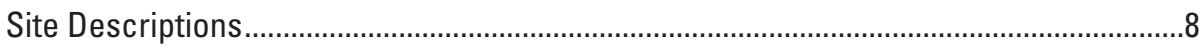

Discrete Water Sample Collection and Analysis...........................................................16

High-Frequency Water-Quality Data Collection .............................................................21

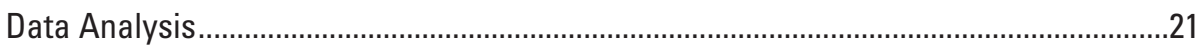

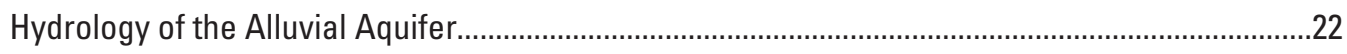

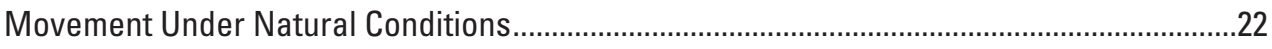

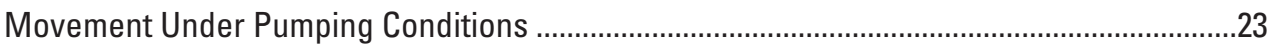

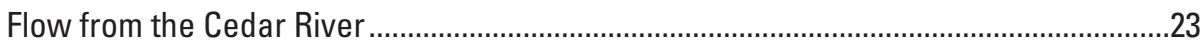

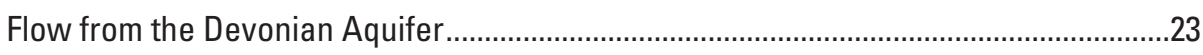

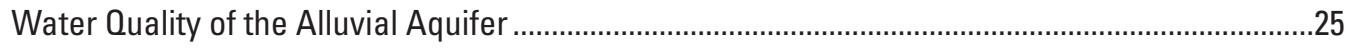

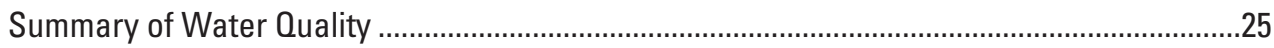

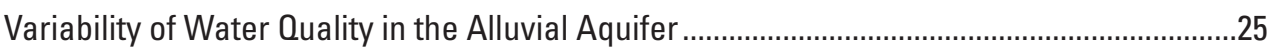

Water-Quality Differences Caused by Well Type .........................................................25

Spatial Variability in Alluvial Water Quality ....................................................................33

Temporal Variability in Alluvial Water Quality .................................................................36

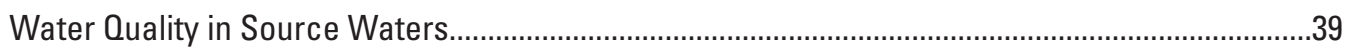

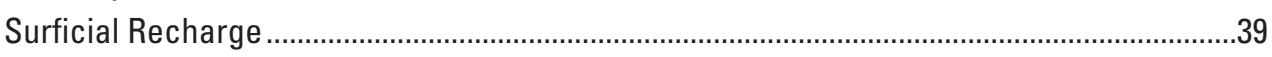

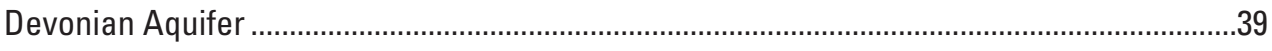

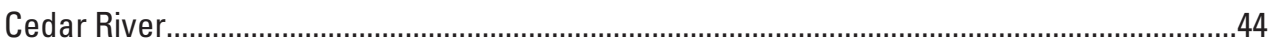

Relation Between Water Quality of the Alluvial Aquifer and the Devonian Aquifer .......................49

Relation Between Water Quality of the Alluvial Aquifer and the Cedar River..................................49

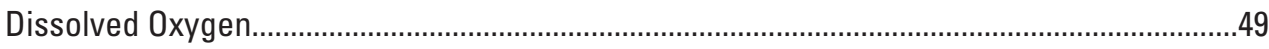

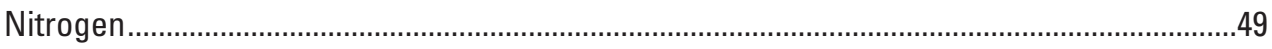

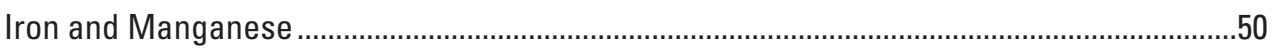

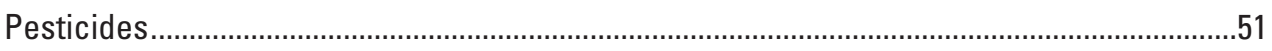

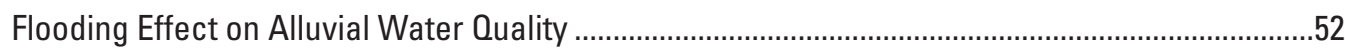


Appendix 1. Pesticide Compounds Not Detected in the Cedar River Alluvial and Devonian Aquifers and the Cedar River near Cedar Rapids, Linn County, lowa, 1990-2019

\section{Figures}

1. Map showing study area, extent of the alluvial aquifer, and surface-water sampling sites in the Cedar River Basin, Linn County, lowa.

2. Graphs showing annual mean precipitation in Cedar Rapids, lowa, and annual mean streamflow in the Cedar River at Cedar Rapids, Linn County, lowa, 1990-2019 .....5

3. Lithological section at three representative monitoring wells completed in the Devonian aquifer in the Cedar Rapids well fields, Linn County, lowa

4. Conceptual model of flow in shallow groundwater near Cedar River in Linn County, lowa.

5. Map showing production and monitoring wells in the City of Cedar Rapids, Linn County, lowa, in three well fields that were monitored during the 1990-2019 study period

6. Conceptual flow through the Cedar River alluvial aquifer, Linn County, lowa 22

7. Simulated flow in the alluvial aquifer caused by pumping from a large-capacity production well

8. Graph showing water level in the alluvial aquifer in relation to the water level in the Cedar River, Linn County, lowa, March 1993 through December 1993

9. Boxplots showing comparison of water chemistry in relation to well type and potential source water to the alluvial aquifer, Linn County, lowa, 1990-2019.

10. Graphs showing detected pesticide compounds from Ranney and vertical production wells and monitoring wells screened in the Cedar River alluvial aquifer near Cedar Rapids, Linn County, lowa, 2003-19

11. Graphs showing change in alluvial water quality along a reach of the Cedar River through Cedar Rapids for Ranney production wells in the Seminole well field in Linn County, lowa, 2003-19

12. Boxplots showing spatial variability in nitrate plus nitrite nitrogen, ammonia as nitrogen, iron, and manganese concentrations in samples from Cedar Rapids production wells, Linn County, lowa, May 2012, from upstream to downstream.

13. Graphs showing nitrate plus nitrite nitrogen and manganese concentrations in monitoring well CRM22, Linn County, lowa, 1998-2010

14. Graphs showing trends in selected water-quality constituents in the alluvial aquifer and in the Cedar River near Cedar Rapids, Linn County, lowa, 1990-2019 .........37

15. Graphs showing trend in concentrations of the four most commonly detected pesticide compounds in the Cedar River alluvial aquifer and the Cedar River in

Linn County, lowa, 1990-2019

16. Graphs showing temporal trend in water quality in the Devonian aquifer Cedar Rapids Seminole well field, Linn County, lowa, 1990-2019.

17. Graph showing number of days the maximum contaminant level for nitrate plus nitrite nitrogen in drinking water was exceeded in the Cedar River near Palo,

Linn County, lowa, 1990-2019 
18. Graph showing water-level altitude and dissolved oxygen concentration in the Cedar River and vertical production well Sem10, Linn County, lowa, December 1993.

19. Graphs showing relation between nitrate plus nitrite nitrogen concentration in the alluvial aquifer and the Cedar River, Linn County, lowa, 2015-16. . .51

20. Graphs showing nitrate plus nitrite nitrogen concentration in floodwaters of the Cedar River, Linn County, lowa.

\section{Tables}

1. Wells monitored for water levels and water quality, Linn County, lowa, 1990-2019.......10

2. Sites on the Cedar River monitored for stage, flow, and water quality, Linn County, lowa, 1990-2019.

3. Carbon, nutrients, major ions, dissolved solids, and physical characteristics analyzed for in water-quality samples, Linn County, lowa, 1990-2019

4. Selected pesticides, degradates, and intermediates analyzed for in water-quality samples, Cedar Rapids, Linn County, lowa, 1990-2019

5. Hydrologic gradient between the Cedar River alluvial aquifer and underlying Devonian aquifer in the well fields in Cedar Rapids, Linn County, lowa, 1994-2001

6. Summary of the physical properties, major ions, nutrients, and trace elements in the alluvial aquifer in the vicinity of Cedar Rapids, Linn County, lowa, 1990-2019 .....26

7. Occurrence of pesticide compounds in the alluvial aquifer, Devonian aquifer, and Cedar River near Cedar Rapids, Linn County, lowa, 1990-2019

8. Summary of the physical properties, major ions, nutrients, and trace elements in relation to well type in the alluvial aquifer near Cedar Rapids, Linn County, lowa, 1990-2019

9. Summary of the physical properties, major ions, nutrients, and trace elements in forested and agricultural recharge areas of the alluvial aquifer near Cedar Rapids, Linn County, lowa, 1996-2003.

10. Occurrence of selected pesticides in forested and agricultural recharge areas of the Cedar River alluvial aquifer in Linn County, lowa, 1996-2003

11. Summary of the physical properties, major ions, nutrients, and trace elements in the Devonian aquifer near Cedar Rapids, Linn County, lowa, 1990-2019

12. Summary of the physical properties, major ions, nutrients, and trace elements in Devonian aquifer near Cedar Rapids, Linn County, lowa, 1993-97

13. Summary of the physical properties, major ions, nutrients, and trace elements in the Cedar River near Cedar Rapids, Linn County, lowa, 1990-2019.

14. Median values of physical properties, major ions, nutrients, and trace elements in the Cedar River during normal and flood conditions, Linn County, lowa, 1990-2019 


\section{Conversion Factors}

U.S. customary units to International System of Units

\begin{tabular}{lll}
\hline \multicolumn{1}{c}{ Multiply } & \multicolumn{1}{c}{ By } & \multicolumn{1}{c}{ To obtain } \\
\hline inch (in.) & \multicolumn{1}{c}{ Length } \\
foot (ft) & 2.54 & centimeter $(\mathrm{cm})$ \\
mile (mi) & 0.3048 & meter $(\mathrm{m})$ \\
\hline & 1.609 & kilometer $(\mathrm{km})$ \\
\hline square mile $\left(\mathrm{mi}^{2}\right)$ & Area & \\
\hline & 2.590 & square kilometer $\left(\mathrm{km}^{2}\right)$ \\
\hline cubic foot per second $(\mathrm{ft} 3 / \mathrm{s})$ & Flow rate & cubic meter per second $\left(\mathrm{m}^{3} / \mathrm{s}\right)$ \\
million gallons per day $(\mathrm{Mgal} / \mathrm{d})$ & 0.02832 & cubic meter per second $\left(\mathrm{m}^{3} / \mathrm{s}\right)$ \\
\hline
\end{tabular}

Temperature in degrees Fahrenheit $\left({ }^{\circ} \mathrm{F}\right)$ may be converted to degrees Celsius $\left({ }^{\circ} \mathrm{C}\right)$ as follows:

$$
{ }^{\circ} \mathrm{C}=\left({ }^{\circ} \mathrm{F}-32\right) / 1.8 \text {. }
$$

\section{Datum}

Vertical coordinate information is referenced to the North American Vertical Datum of 1988 (NAVD 88), unless otherwise stated.

Horizontal coordinate information is referenced to the North American Datum of 1983 (NAD 83).

Altitude, as used in this report, refers to distance above the vertical datum.

\section{Supplemental Information}

Specific conductance is given in microsiemens per centimeter at 25 degrees Celsius $(\mu \mathrm{S} / \mathrm{cm}$ at $\left.25^{\circ} \mathrm{C}\right)$.

Concentrations of chemical constituents in water are given in either milligrams per liter (mg/L) or micrograms per liter $(\mu \mathrm{g} / \mathrm{L})$. 


\section{Abbreviations}

$\begin{array}{ll}> & \text { greater than } \\ < & \text { less than } \\ \text { ammonia-N } & \text { ammonia as nitrogen } \\ \text { CEAT } & \text { atrazine degradate [2-chloro-6-ethylamino-4-amino-s-triazine] } \\ \text { CIAT } & \text { atrazine degradate [2-chloro-4-isopropylamino-6-amino-s-triazine] } \\ \text { DOC } & \text { dissolved organic carbon } \\ \text { EPA } & \text { U.S. Environmental Protection Agency } \\ \text { IFIS } & \text { lowa Flood Information System } \\ \text { MCL } & \text { maximum contaminant level } \\ \text { nitrate-N } & \text { nitrate plus nitrite nitrogen } \\ \text { NOAA } & \text { National Oceanic and Atmospheric Administration } \\ p & \text { probability value } \\ \text { redox } & \text { reduction-oxidation } \\ \text { SMCL } & \text { secondary maximum contaminant level } \\ \text { USGS } & \text { U.S. Geological Survey }\end{array}$





\title{
Hydrologic and Water-Quality Conditions in the Cedar River Alluvial Aquifer, Linn County, lowa, 1990-2019
}

\author{
By Stephen J. Kalkhoff
}

\section{Abstract}

Alluvial aquifers in Iowa have more wells with nitrate exceeding drinking-water standards than other aquifers; are susceptible to contamination by organic contaminants; and have high concentrations of naturally occurring iron and manganese in depositional areas that contain abundant organic matter. The U.S. Geological Survey, in cooperation with the City of Cedar Rapids, Iowa, studied the Cedar River alluvial aquifer in Linn County, Iowa, from 1990 to 2019 to understand the effect of municipal pumping on spatial and temporal hydrologic and water-quality variability. The Cedar River alluvial aquifer is the source of water for the city of Cedar Rapids, Iowa. Withdrawal of large quantities of water for municipal and industrial supply has altered the normal flow of water in the alluvial aquifer. Pumping induces flow from the Cedar River and the underlying bedrock aquifer into the alluvial aquifer.

Water quality in the alluvial aquifer varies along the Cedar River. Changes in nitrate, ammonia, manganese, and iron in the alluvial aquifer are seen as the upstream freeflowing reach of the Cedar River transitions to a partially regulated downstream reach, likely because of differences in reduction-oxidation conditions in the aquifer, which are controlled by infiltration from the Cedar River under normal conditions and when wells are being pumped. Nitrate, normally found in oxygenated environments, had the highest concentrations in the most upstream wells in the Seminole well field and the lowest concentrations in the most downstream wells in the East well field. In contrast, ammonia, manganese, and iron, normally found in greatest abundance in anoxic (reducing) conditions, had the greatest concentrations in the most downstream wells. Additionally, dissolved nitrate plus nitrite nitrogen concentrations in wells were substantially less and manganese concentrations were greater in production wells near backwater wetlands in contrast to wells near the Cedar River.

Temporal variability in water quality in the alluvial aquifer was driven by pumping that increased flow from the Cedar River into the alluvial aquifer and ultimately led to changes in reduction-oxidation conditions of the aquifer. Increasing dissolved nitrate plus nitrite nitrogen concentrations in the Cedar River from 1990 to 2019 were mirrored in the alluvial aquifer.
Anoxic conditions are prevalent in the alluvial aquifer next to the Cedar River when the aquifer is not under pumping stress. However, production well pumping caused induced infiltration of oxygenated river water into the aquifer resulting in increased dissolved nitrate plus nitrite nitrogen concentrations and pesticides and decreased naturally occurring dissolved iron and manganese.

Hydrologic and water-quality conditions in the Cedar River alluvial aquifer from 1990 to 2019 provide baseline conditions needed to evaluate the effects of current and future nutrient reduction efforts and land-use changes in the Cedar River Basin on water quality of the Cedar River alluvial aquifer and its source water, the Cedar River. This summary and analysis provide information that can assist the City of Cedar Rapids Utilities Water Department in managing groundwater resources, and provides information that could be used develop a groundwater-quality model to characterize variability over larger areas of the alluvial aquifer, allowing water providers to plan for future water needs of their users.

\section{Introduction}

Sand and gravel deposits along rivers and streams (surficial alluvial aquifers) are an important source of water for residential and municipal water supply in Iowa. These deposits can be as much as 100 feet (ft) thick and provide more than 50 percent of the water withdrawn from groundwater in Iowa (Clarke and Thamke, 1988). The unconsolidated surficial aquifers are readily recharged from infiltration of rainfall and from natural (Squillace, 1996) and induced inflow (Boyd, 1998; Turco and Buchmiller, 2004) from the adjacent river or stream. Because alluvial aquifers are readily recharged from the land surface and from nearby rivers and streams, they are susceptible to contamination. Alluvial aquifers in Iowa have more wells with nitrate plus nitrite nitrogen (nitrate-N) concentrations exceeding drinking-water standards than other aquifers (Schaap and Linhart, 1998). Alluvial aquifers also are susceptible to organic contamination (Squillace and others, 1996; Savoca and others, 2000). High concentrations of naturally occurring iron and manganese are present in parts of the alluvial aquifers in depositional areas that contain abundant organic matter (Schulmeyer and Schnoebelen, 1998). 
The Cedar River alluvial aquifer in Linn County, Iowa, is the source of water for the city of Cedar Rapids. The Cedar Rapids Utilities Water Department pumps and treats between 30 and 50 million gallons per day (Dustin Elin, Cedar Rapids Utilities Process Control Specialist, written commun., 2020) for home and industrial use. Several anthropogenic and naturally occurring constituents in water from the alluvial aquifer and in the Cedar River are of concern because they may exceed U.S. Environmental Protection Agency (EPA) maximum contaminant levels (MCLs) or secondary drinking-water standards. These constituents include excess nitrogen and pesticides that originate from point and nonpoint sources in the Cedar River Basin and naturally occurring trace elements, iron and manganese, that originate from the alluvial deposits.

Runoff from the Cedar River Basin has contributed additional nitrogen, phosphorus (Kalkhoff, 2018), and organic pesticides (Squillace and Engberg, 1988) to the Cedar River. The Cedar River is designated for recreation, as a warm-water resource, and as a drinking-water supply (Iowa Department of Natural Resources, 2006). Excess nitrogen in the Cedar River impairs use for drinking water, which caused the river to receive a 303(d) listing for high nitrate- $\mathrm{N}$ concentrations (Iowa Department of Natural Resources, 2006). The impaired reach extends from the well fields in Cedar Rapids along the Cedar River upstream to the city of Palo, Iowa (Iowa Department of Natural Resources, 2006).

\section{Description of the Problem}

Studies on the movement of water and water quality in the alluvial aquifer, the underlying bedrock aquifer, and the Cedar River and on how they interact have been ongoing since the early 1990s. Results of these studies have been published in several reports, but there is a need to compile these historical data and results with recent information to better document water quality in the Cedar River alluvial aquifer in Linn County, Iowa. Specifically there is a need to (1) characterize water quality in the alluvial aquifer and its source waters, (2) document changes in water quality that may have occurred since the early 1990s, (3) describe the relation between water quality in the alluvial aquifer and water quality in recharge from deeper bedrock aquifers and the Cedar River, and (4) understand geochemical changes in the aquifer that will likely affect the City's water supply. This analysis provides background data that can be used to develop a future groundwater-quality model that can characterize variability over larger areas of the alluvial aquifer and ultimately allow water providers to plan for future supplies. Information also is needed for development of additional water supplies as the anticipated demand for water increases as city of Cedar Rapids continues to expand. Tools, including groundwater-quality models, may be needed to plan for the development of groundwater resources as land use in and near the city and upstream in the Cedar River Basin continue to change.
Hydrologic and water-quality data are needed to form the basis on which coordinated nutrient reduction practices upstream in the Cedar River Basin currently being led by the City of Cedar Rapids Middle Cedar Partnership Project (Cedar Rapids, 2020) along with the Middle and Upper Cedar River Watershed Management Authorities (Iowa Department of Natural Resources, 2020c) and the University of Iowa Hydrologic Research Iowa Watershed Approach (Iowa Watershed Approach, 2020) can be evaluated. Implementation of future practices are anticipated as part of the Iowa Nutrient Reduction Strategy (Iowa State University, 2014).

\section{Previous Investigations}

Early documentation of water quality from deep bedrock wells in Cedar Rapids occurred in 1912 (Norton and others, 1912) and in the early 1930s (Iowa State Planning Board, 1938). One of the earliest reports describing the lithology, water movement, and water quality of the alluvial aquifer in Linn County, Iowa, was by Hansen (1970). Information on water resources in Linn County in the 1970s were provided by Wahl and others (1978) in a regional atlas on the occurrence, availability, quality, and use of water in east-central Iowa.

A series of reports (Wang and Squillace, 1994; Squillace, 1996, Squillace and others, 1996) documented the movement of water and agricultural chemicals between the Cedar River and the adjacent alluvial aquifer at an unfarmed site about 12 miles downstream from well fields in Cedar Rapids. Studies on the movement of water and water quality in the alluvial aquifer and the interaction of water in the alluvial aquifer and the Cedar River have been ongoing since the early 1990s in cooperation with the City of Cedar Rapids. Previous reports documenting the results of these studies are summarized below.

Schulmeyer (1995) documented the filtering capacity of the alluvial aquifer in relation to biogenic material in the Cedar River. No Giardia spp. (Kunstler, 1882) cysts or Cryptosporidium spp. (Tyzzer, 1907) oocysts were present in water collected from production wells. The aquifer is filtering out large numbers of algae, diatoms, rotifers, and nematodes, as well as filtering out Cryptosporidium, Giardia, and other protozoa. The number of algae, diatoms, rotifers, protozoa, and vegetative debris for selected production wells tested was reduced to at least 1 per 1,000 of the number measured in the river. The filtering efficiency of the aquifer is equivalent to a three logarithmic-reduction rate or a 99.99-percent reduction in particulates.

Boyd (1998) used stable isotopes, tritium, and chlorofluorocarbons to determine that water in the bedrock aquifer likely was recharged before the 1950s, and water in the alluvial aquifer likely was recharged within the last 30 years. The relative amount of water contributed to the production well Seminole- 10 by the alluvial aquifer, bedrock aquifer, and the Cedar River was 49 percent, 5 percent, and 46 percent, respectively.

Schulmeyer and Schnoebelen (1998) associated large dissolved-iron and dissolved-manganese concentrations with areas near old meander channels and wetlands. These depositional 
areas contain abundant organic matter, which decomposes and can reduce conditions favorable to the dissolution of minerals containing oxidized forms of iron and manganese.

Boyd (1999) determined that microbial-catalyzed reductionoxidation (redox) reactions altered the chemical composition of Cedar River water infiltrating into the alluvial aquifer by consuming dissolved oxygen, reducing nitrate- $\mathrm{N}$, and increasing dissolved iron and manganese concentrations. Nitrate- $\mathrm{N}$ reduction only occurred in relatively shallow groundwater (10 to $23 \mathrm{ft}$ below land surface) near the Cedar River and did not occur in water infiltrating to deeper zones of the alluvial aquifer.

Boyd (2000) evaluated the occurrence of herbicides and herbicide degradates in production wells during a period after springtime application of herbicides in the Cedar River Basin. Atrazine was most frequently detected and occurred at the greatest concentrations; acetochlor, cyanazine, and metolachlor also were detected but at lesser concentrations than atrazine.

Schnoebelen (2008) determined that alluvial wetlands may provide substantial reductions of nitrate- $\mathrm{N}$ concentrations in groundwater and may be a useful strategy for reducing nitrate for production wells. However, reduced nitrate- $\mathrm{N}$ concentrations may be offset by increased dissolved iron and manganese concentrations. Wetlands had negligible effect on pesticide concentrations.

Three groundwater-flow models were developed to better understand the groundwater flow system of the Cedar River and the alluvial aquifer near well field areas. The first groundwater model that included the alluvial aquifer in the Cedar Rapids area was regional in scope (231 square miles [ $\left.\mathrm{mi}^{2}\right]$ ) and was described by Schulmeyer and Schnoebelen (1998). This model, which had a 500- by 500-ft grid size, indicated that the primary sources of inflow to the alluvial aquifer included infiltration from the Cedar River (74.2 percent), infiltration from underlying hydrogeologic units (20.9 percent), and infiltration from precipitation (4.9 percent). Pumpage by large-capacity production wells from the alluvial aquifer accounted for 78 percent of the system outflow. The second, more-detailed model includes a $45-\mathrm{mi}^{2}$ area centered around the well fields in Cedar Rapids (Turco and Buchmiller, 2004). Grid sizes as small as 50 - by 50 -ft within the well field were used to better define flow to individual wells. The model was intended to help in the detailed study of the surface water and groundwater interaction in the well field and to better evaluate the effect of the Cedar River on the quality of water supplies. A third groundwater model was developed when observed drawdowns in the aquifer during a drought in 2012 could not be simulated with the second model (Haj and others, 2021). An enhanced MODFLOW model with more detailed lithologic and hydrologic properties of the aquifer obtained from several geophysical investigations was used to successfully simulate observed water-level declines and better understand the effects of drought stress on the Cedar River alluvial aquifer (Haj and others, 2021). This third-generation model can help the City of Cedar Rapids evaluate aquifer resilience and develop a drought management and response strategy.

\section{Purpose and Scope}

The purpose of this report is to document water quality in the Cedar River alluvial aquifer and other waters that contribute to the Cedar Rapids production wells by summarizing hydrologic and water-quality conditions in the Cedar River alluvial aquifer from 1990 through 2019. The summary includes analysis of temporal and spatial trends and describes how water quality in the aquifer was affected by differences in production well pumping, geochemical conditions, and changes in river water quality. The relations between water quality in the alluvial aquifer, Cedar River, and underlying bedrock aquifers are described. The primary focus is on an understanding of the hydrology and water quality during 1990-2019. Data and findings documented in previous reports and recently collected data are used in this summary (data are available in the U.S. Geological Survey [USGS] data release; Meppelink and Kalkhoff, 2021). Constituents of primary concern in a water supply, such as nutrients (nitrate- $\mathrm{N}$ and ammonia as nitrogen [ammonia-N]), total dissolved solids (including all major anions and cations), dissolved organic carbon (DOC), common pesticides, and the trace elements iron and manganese are included in the analysis. The alluvial aquifer from Palo, Iowa, to Cedar Rapids, Iowa, is included in the study area.

\section{Description of the Study Area}

The Cedar River alluvial aquifer study area is in the south-central part of the Cedar River Basin (fig. 1, inset map). The Cedar River upstream from the study area drains an area of 6,340 $\mathrm{mi}^{2}$ that is used primarily for agriculture. More than 80 percent of the basin is used for corn and soybean production but includes some pastureland, hay, and small grains (Iowa Department of Natural Resources, 2006). Cedar Rapids is the largest city in the basin, but other cities with similar population, including Waterloo in Iowa, and Albert Lea and Austin in Minnesota, are upstream from Cedar Rapids, Iowa (not shown).

\section{Climate}

The climate in the study area is continental. Air temperatures in Cedar Rapids, Iowa, varied from year to year during the 1990-2019 study period; the annual mean air temperature ranged from 47.1 degrees Fahrenheit $\left({ }^{\circ} \mathrm{F}\right)$ in 2014 to $54.1^{\circ} \mathrm{F}$ in 2012. The annual mean in 2012 was the greatest in the more than 120 years of record at the Cedar Rapids weather station (National Oceanic and Atmospheric Association [NOAA], 2019). One of the coolest years (2014) on record had an annual mean temperature of $47.1^{\circ} \mathrm{F}$ (NOAA, 2019). Overall, there was very little difference between the mean annual air temperature during the study period and the 1981-2010 30-year mean (NOAA, 2019). 


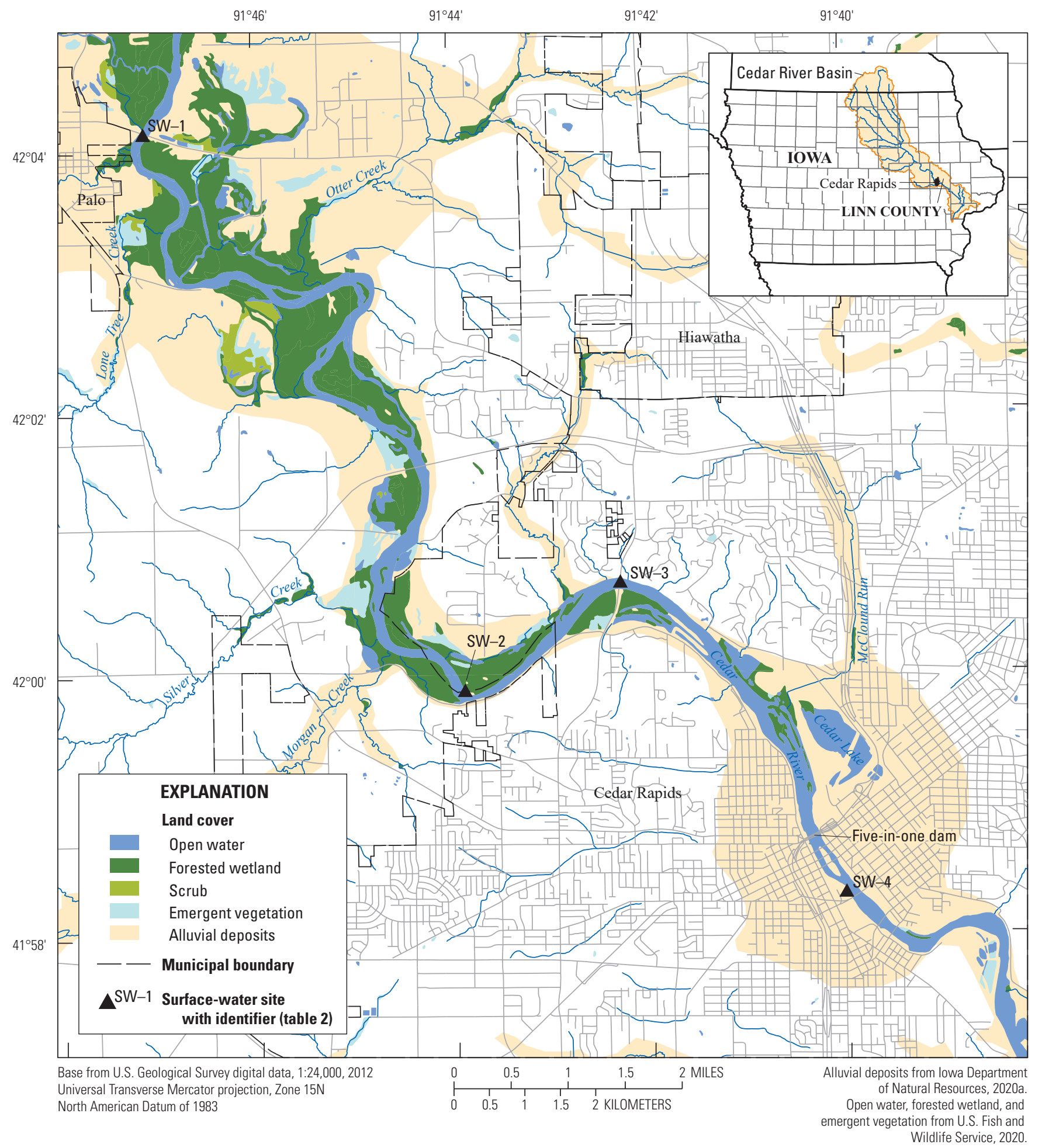

Figure 1. Study area, extent of the alluvial aquifer, and surface-water sampling sites in the Cedar River Basin, Linn County, lowa. 
Precipitation in Cedar Rapids, Iowa, was highly variable (fig. 2) during the study. The study period included the wettest year (1993) and one of the driest years (2012) of the 128-year NOAA record at the Cedar Rapids weather station (NOAA, 2019). Annual precipitation ranged from 60.12 inches (in.) in 1993 to 25.58 in. in 2012. Annual precipitation during the first half of the study period was generally in the normal (25th to 75th percentile) to below-normal range, except for 3 years with greater than normal precipitation. One of these years (1993) was the annual maximum precipitation during the study period. During the second half of the study period, precipitation was generally in the normal to greater than normal range. Precipitation was below normal only in 2006 and 2012. The median precipitation during the study period was 37.77 in., which was essentially the same as the 1981-2010 30-year mean of 37.59 in. (NOAA, 2019). Rainfall is normally the greatest during May and June.

\section{Streamflow}

Annual mean streamflow in the Cedar River at Cedar Rapids (fig. 2) was variable during the 1990-2019 period and reflected the variability in annual precipitation in the Cedar River Basin. Streamflow during the 1990-2019 period had a mean of 6,380 cubic feet per second $\left(\mathrm{ft}^{3} / \mathrm{s}\right)$ and ranged from 2,050 ft $3 / \mathrm{s}$ in 2012 to $15,100 \mathrm{ft}^{3} / \mathrm{s}$ in 1993 (site 05464500; USGS, 2020). Eight of the 10 years with the greatest annual mean streamflow during the 118-year record at Cedar Rapids occurred during the study period. Streamflow in the Cedar River was much greater than normal in the last decade of the study period because 5 of the 10 years with the greatest annual mean streamflow occurred in 2010 or later. Greater than normal precipitation in the latter period of the study resulted in annual mean streamflow that increased substantially from the early 2000s through the end of the study period (fig. 2).

\section{Description of the Alluvial Aquifer}

The alluvial aquifer is contained in alluvial deposits and is present in the valley floor of the Cedar River and ranges from about 2,000 ft wide in the well fields in Cedar Rapids to more than 3 miles wide near Palo, Iowa (fig. 1). Uplands next to the alluvial aquifer consist of till and windblown loess. The alluvial aquifer is underlaid by till deposits around extensive alluvial deposits near Palo, Iowa, and by bedrock where alluvial deposits are relatively narrow in the City of Cedar Rapids, Iowa (Valder and others, 2018). The alluvial valley is bounded by steep bluffs of uplands that consist of till and windblown loess (Quade and others, 1998) and include bedrock exposures, and can rise almost $200 \mathrm{ft}$ above the river floodplain (Schnoebelen, 2008).

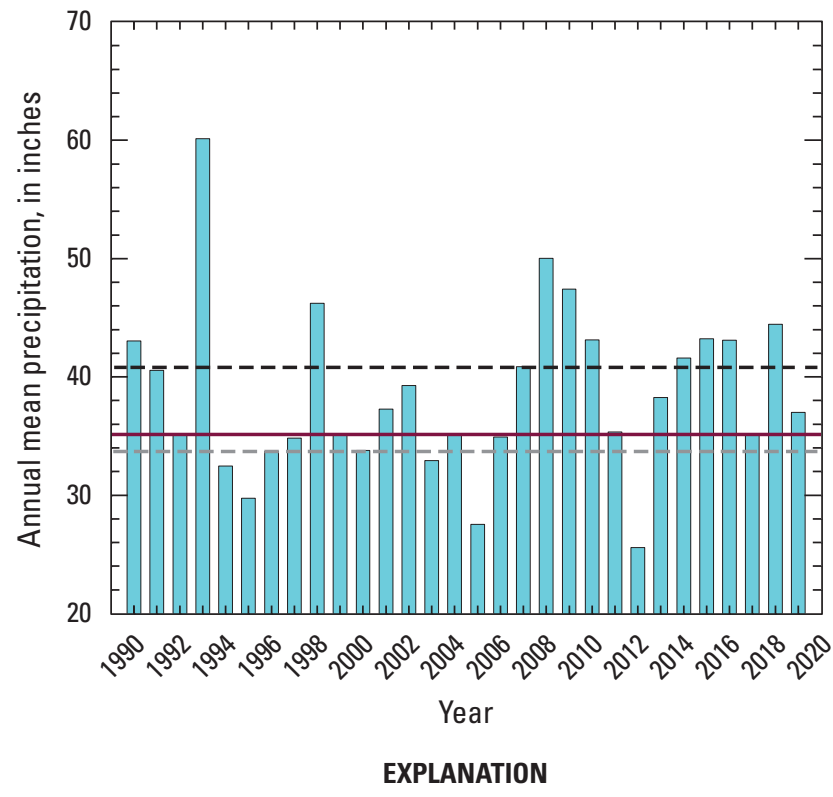

[Data are from the National Oceanic and Atmospheric Administration (2019) Cedar Rapids No 1 station]
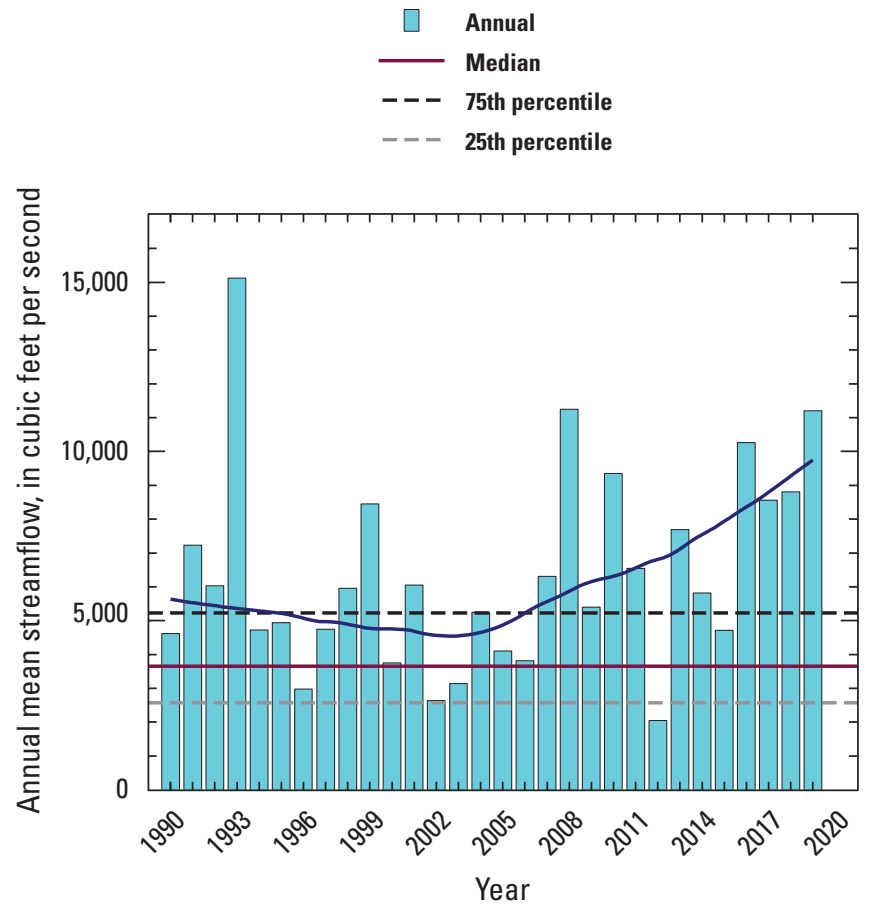

EXPLANATION

[Data are from the U.S. Geological Survey (USGS) National Water Information System database (USGS, 2020) site 05464500]

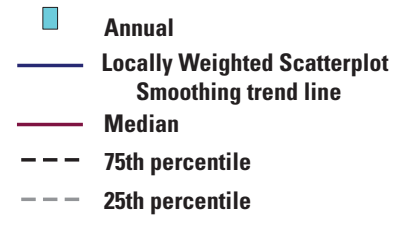

Figure 2. Annual mean precipitation in Cedar Rapids, lowa, and annual mean streamflow in the Cedar River at Cedar Rapids, Linn County, lowa, 1990-2019. 


\section{Land Cover}

Alluvial deposits near Cedar Rapids are covered with an extensive area of backwater ponds and wetlands in the river floodplain. Much of the wetland is forested, but extensive areas of wetland scrub and emergent vegetation are present. Wetlands are characterized by vegetation as forested, deciduous, or broad-leaved deciduous with lower perennial plants or emergent vegetation and are flooded intermittently or seasonally (Schnoebelen, 2008). The soil type is hydric with underlying sand and some silt. Several natural oxbow ponds also are present within the study area. Most of the ponds are connected to the Cedar River, and some ponds are connected intermittently to the Cedar River when streamflow in the Cedar River exceeds about 5,000 ft $3 / \mathrm{s}$ (Schnoebelen, 2008).

\section{Geology}

The Cedar River alluvial deposits consist of a sequence of coarse sand and gravel at the base, grading upwards to finer sand, silt, and clay near the surface that has been deposited by the Cedar River. Eolian sand deposits are present in areas where wind has reworked older alluvial deposits (Schulmeyer and Schnoebelen, 1998; Quade and others, 1998). The sand and gravel may contain carbonate, shale, and ferro-magnesium-rich rock fragments (Schnoebelen, 2008). The thickness of alluvial deposits ranges from less than $1 \mathrm{ft}$ next to the valley walls to greater than $90 \mathrm{ft}$ along the Cedar River. The thickness is variable throughout the study area, but the maximum thickness uniformly ranges from about 40 to $80 \mathrm{ft}$ in the upper to lower end of the study area (Valder and others, 2018). Cedar River tributaries have deposits that may be $10 \mathrm{ft}$ or more thick that are connected to the Cedar River alluvial aquifer.

The alluvial deposits overlie bedrock formations consisting of (youngest to oldest) the Cedar Valley Limestone and Wapsipinicon Formation of Devonian age and the Gower Dolomite, Hopkinton Dolomite, Kankakee Formation, and Edgewood Dolomite of Silurian age (Hansen, 1970). The Devonian-aged formations, the shallowest bedrock formations in the study area, consist of limestone, dolomite, and some shale units (Hansen, 1970). The water-bearing Devonian-aged formations contain what is referred to as the Devonian aquifer in the remainder of this report. A shale unit at the top of the Devonianaged formations was identified in drillers lithologic logs from a monitoring well (CRM6A) in the Seminole well field (fig. 3). The shale overlies limestone deposits containing shale stringers. The shale layer was missing farther east in the West (CRM11) and East (CRM12) well fields. The limestone with shale stringers that lie directly below the unconsolidated sands and gravel of the alluvial deposits (fig. 3) in the West and East well fields are Devonian-aged formations.

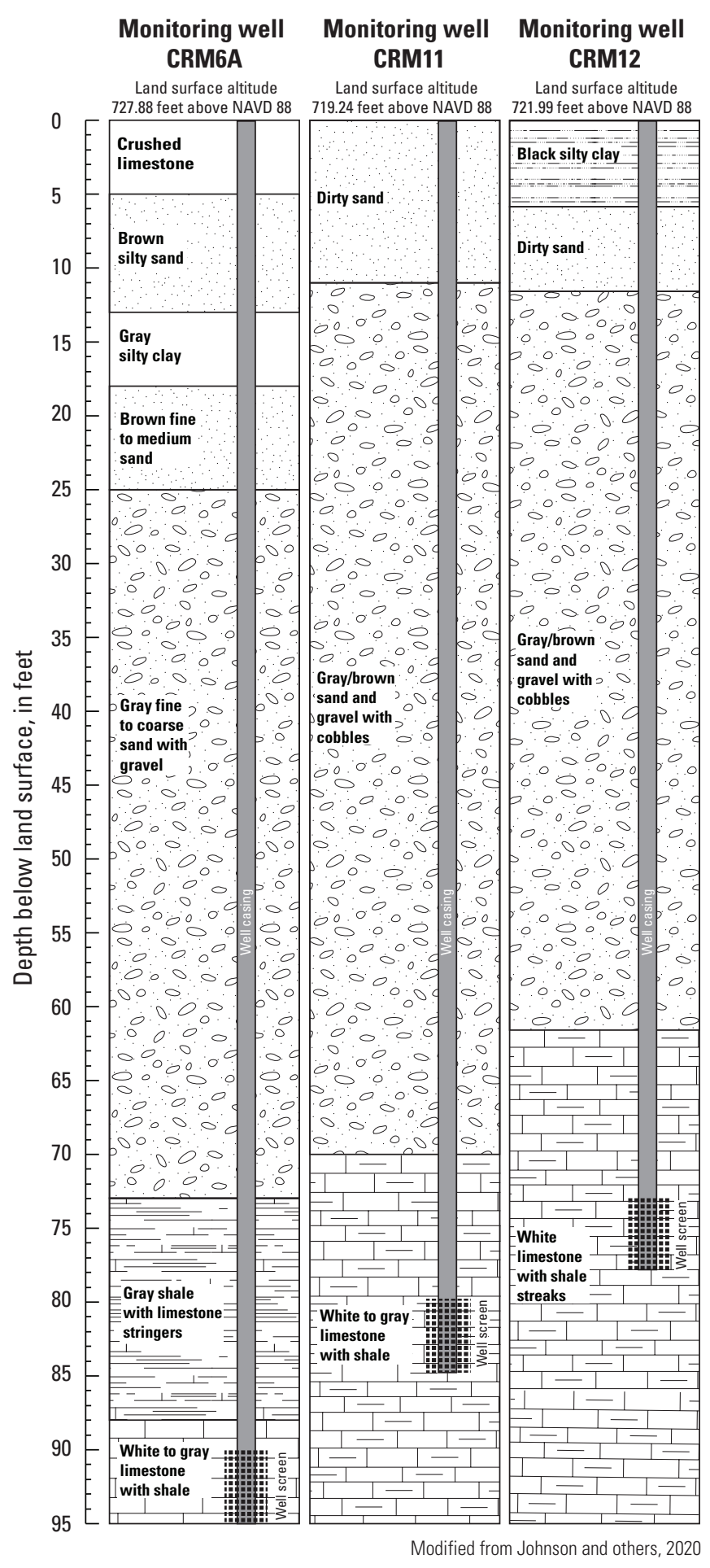

Figure 3. Lithological section at three representative monitoring wells completed in the Devonian aquifer in the Cedar Rapids well fields, Linn County, lowa. 


\section{Movement of Water}

The alluvial aquifer is part of a complex hydrologic system where water moves between several unconsolidated and bedrock formations and the Cedar River (fig. 4). Fine- to course-grained sands of the alluvial aquifer are recharged by infiltration of rainfall and snowmelt through surficial sediment and water moving laterally from the adjacent weathered and unweathered till and eolian loess deposits. Water moves laterally through the alluvial aquifer to the Cedar River. When the level of the Cedar River is high, the hydrologic gradient is reversed such that water from the Cedar River and floodplain backwaters and wetlands infiltrates through the streambank and streambed into the alluvial aquifer (Squillace, 1996). Regional flow in the deeper carbonate bedrock Devonian-Silurian aquifer may move into the alluvial aquifer. Water moves primarily to the Cedar River but at times may move downward into the underlying Devonian bedrock aquifer. Flow into and out of the alluvial aquifer from the Devonian aquifer may be impeded by a shale layer in the upstream part of the study area (Hansen, 1970). The natural flow path in the alluvial aquifer can be altered by pumping to supply residential and industrial purposes (Turco and Buchmiller, 2004; Boyd, 1998; Schulmeyer, 1995).

\section{Water Use}

Water from the alluvial aquifer is heavily used by the City of Cedar Rapids, Iowa, for residential and industrial purposes. The City pumps water from three well fields that stretch about 6 miles mainly along the left or north bank of the Cedar River upstream from the five-in-one low head dam to about 2 miles south of U.S. Highway 100 (figs. 1 and 5). The water level of the pool upstream from the dam is maintained at an altitude of $717.97 \mathrm{ft}$ above sea level (Tariq Baloch, Cedar Rapids Utilities Water Department, written commun., 2019). As of 2020, water is obtained from 49 vertical production wells and 5 horizontal collector wells (called "Ranney production wells" herein) (Meppelink and Kalkhoff, 2021).

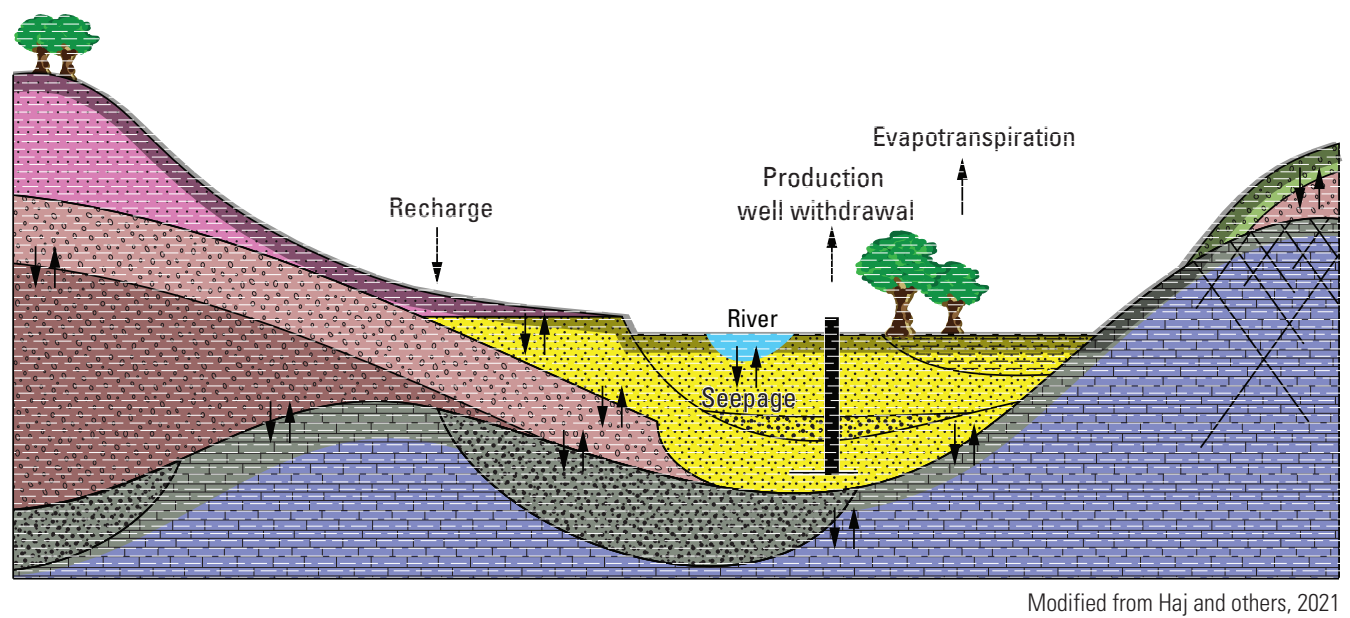

\section{EXPLANATION}

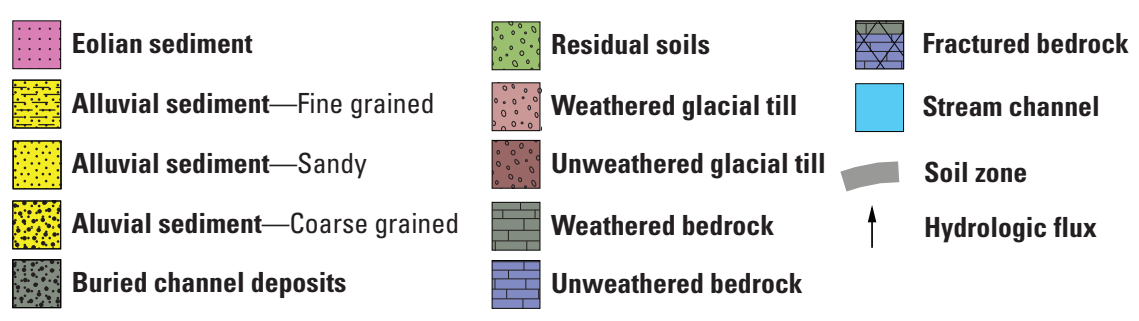

Figure 4. Conceptual model of flow in shallow (less than 100 feet) groundwater near Cedar River in Linn County, lowa. 
Hydrologic and Water-Quality Conditions in the Cedar River Alluvial Aquifer, Linn County, lowa, 1990-2019

\section{Methods}

Hydrological and water-quality data were collected during 1990-2019 to document movement of water and the quality of water in alluvial aquifers and other waters that contribute water supplying production wells. Hydrologic data consisted of water levels in the alluvial and underlying Devonian bedrock aquifer and altitude of the water surface and streamflow in the Cedar River. Physical properties, major ions, nutrients, pesticides, and selected trace elements were measured and analyzed in water samples to provide data to characterize water quality and to document geochemical processes in the alluvial aquifer. Two types of production wells, vertical and horizontal collector (Ranney) wells in the Seminole, West, and East well fields were monitored (fig. 5). In addition, water levels and water samples were obtained from monitoring wells throughout the study area (fig. 5). Data were collected using USGS protocols that are described in detail by Schulmeyer and others (1995), Schnoebelen and Schulmeyer (1996), Boyd and others (1999), Littin and Schnoebelen (2010), Littin (2012), and Meppelink and others (2019); and are summarized below.

\section{Hydrologic Data}

Water-level data were collected from wells and the Cedar River to infer water movement based on groundwater-level gradients in the alluvial and bedrock aquifers and the interaction of groundwater with the Cedar River. Water levels in wells were made using manual measurement methods (Cunningham and Schalk, 2011) in 72 wells during the study period (see table 1 for wells mentioned in this report; see Meppelink and Kalkhoff [2021] for the complete list of wells). Water levels were made using a graduated steel or electrical tape to the nearest $0.01 \mathrm{ft}$. Pressure transducers were used to obtain high-frequency measurements in 11 wells (identified by "continuous water-level data" in table 1) as described by Cunningham and Schalk (2011). Water levels measured with the pressure transducers were recorded by dataloggers at 15 to 60-minute intervals and were checked with periodic manual measurements to verify transducer measurements and correct instrument drift and offsets (Cunningham and Schalk, 2011).

Water levels (stage) were measured at three sites (identified by "real-time water level" in table 2) on the Cedar River using nonsubmersible pressure transducers as the pressure sensor for bubble-gage systems (Sauer and Turnipseed, 2010). Flow in the Cedar River entering the study area was calculated from water-level measurements at the Blairs Ferry Road bridge near Palo, Iowa (SW-1), beginning in 2009 (fig. 1, table 2). The river altitude in well fields in Cedar Rapids was measured at site SW-2 during 1993-97 and at the site on Edgewood Road (SW-3) beginning in 2017 (table 2). River water levels were used with stage-discharge ratings to calculate streamflow (Rantz, 1982) at 15-minute intervals at SW-1 and at Cedar Rapids, Iowa (SW-4; table 2). Daily and annual streamflow were then compiled from the 15-minute streamflow data.

\section{Water-Quality Data}

Water samples to characterize the quality of water in the alluvial aquifer and in the Cedar River were collected using USGS protocols (USGS, variously dated). Detailed descriptions of sampling methods are in the 5-year data reports (Schulmeyer and others, 1995; Schnoebelen and Schulmeyer, 1996; Boyd and others, 1999; Littin and Schnoebelen, 2010; Littin, 2012; Meppelink and others, 2019) and are summarized below. Data were collected as part of long-term monitoring to document water-quality changes and as part of short-term or localized studies to understand hydrologic and geologic factors that affect water quality in the alluvial aquifer.

\section{Site Descriptions}

Samples to characterize quality of the alluvial aquifer were collected from the aquifer and from potential source waters that include the underlying Devonian aquifer and the Cedar River. Samples were collected from production and monitoring wells screened in the alluvial aquifer that potentially represent different hydrologic conditions. Production wells were sampled to characterize raw water from the alluvial aquifer used by the City of Cedar Rapids, Iowa. Smalldiameter monitoring wells were installed to obtain data from localized parts of the aquifer.

Water-quality data were obtained by sampling production and monitoring wells screened in the alluvial aquifer (fig. 5). Data from different well types were used to evaluate the overall quality of water from the aquifer, but samples from different well types are generally intended for different purposes. Samples from production wells are used to document the source waters used by the City of Cedar Rapids. Samples from monitoring wells are generally used to document the quality in specific locations or hydrologic settings. Water-quality samples were collected from two types of production wells: vertical production wells from the Seminole, West, and East well fields that are generally screened through the entire depth of the aquifer; and Ranney production wells in the Seminole and West fields that have multiple screens extending horizontally from a central caisson. Additional samples were collected from dedicated monitoring wells (these wells have "CRM" in their site names; fig. 1; table 1; Meppelink and others, 2019) that were installed to monitor the water in the aquifer flowing from the Cedar River to production wells, in wetland areas, and in areas receiving recharge from precipitation. Additional small diameter temporary monitoring wells (these wells have "CRM-SD" in their site names; Meppelink and others, 2019) were installed on the bank of the Cedar River in a line perpendicular to the river to understand the biological and geochemical processes that alter river water as it infiltrates into the 
$91^{\circ} 44^{\prime}$

$91^{\circ} 43^{\prime}$

$91^{\circ} 42^{\prime}$

$91^{\circ} 41$

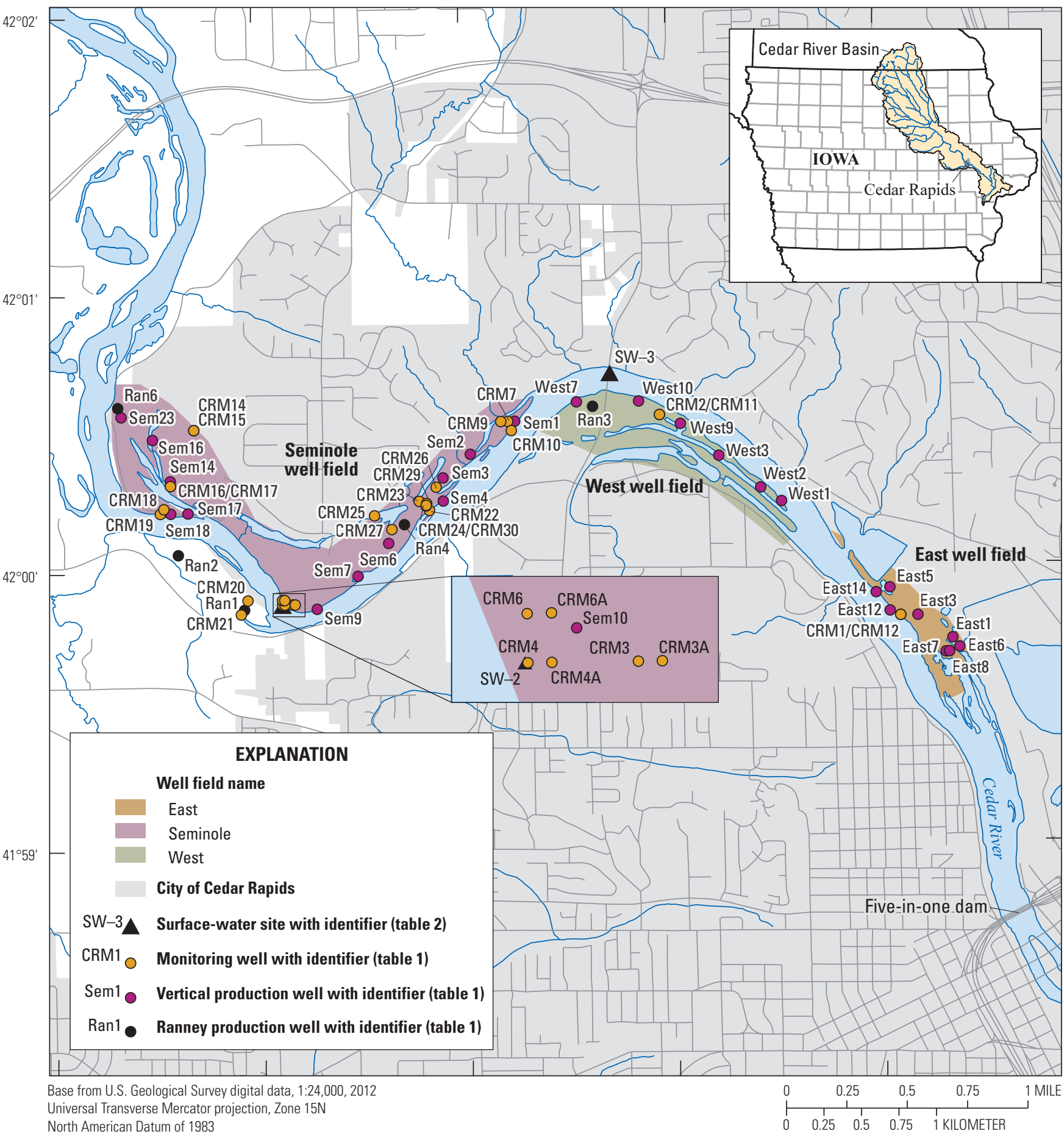

Figure 5. Production and monitoring wells in the City of Cedar Rapids, Linn County, lowa, in three well fields that were monitored during the 1990-2019 study period. 
Table 1. Wells monitored for water levels and water quality, Linn County, lowa, 1990-2019.

[See Meppelink and Kalkhoff (2021) for the complete list of wells monitored during this study; only wells mentioned in this report are listed here. Dates are given in month/day/year. USGS, U.S. Geological Survey; ft, foot; M, monitoring; RP, Ranney production; VP, vertical production; --, not applicable]

\begin{tabular}{|c|c|c|c|c|c|c|c|c|c|c|c|}
\hline \multirow{2}{*}{ USGS site number ${ }^{1}$} & \multirow{2}{*}{ USGS site name ${ }^{1}$} & \multirow{2}{*}{$\begin{array}{c}\text { Site short } \\
\text { name (fig. 5) }\end{array}$} & \multirow{2}{*}{$\begin{array}{l}\text { Well } \\
\text { type }\end{array}$} & \multirow{2}{*}{ Latitude $^{1}$} & \multirow{2}{*}{ Longitude $^{1}$} & \multirow{2}{*}{$\begin{array}{c}\text { Well } \\
\text { depth' } \\
\text { (ft) }\end{array}$} & \multicolumn{2}{|c|}{$\begin{array}{l}\text { Period of water quality } \\
\text { record }{ }^{1}\end{array}$} & \multicolumn{2}{|c|}{$\begin{array}{c}\text { Period of water level } \\
\text { record }{ }^{1}\end{array}$} & \multirow{2}{*}{$\begin{array}{c}\text { Continuous } \\
\text { water-leve } \\
\text { data }\end{array}$} \\
\hline & & & & & & & $\begin{array}{c}\text { Beginning } \\
\text { date }\end{array}$ & $\begin{array}{l}\text { Ending } \\
\text { date }\end{array}$ & $\begin{array}{c}\text { Beginning } \\
\text { date }\end{array}$ & $\begin{array}{c}\text { Ending } \\
\text { date }\end{array}$ & \\
\hline \multicolumn{12}{|c|}{ Alluvial aquifer } \\
\hline 415949091405401 & $\begin{array}{l}\text { 083N07W17DACC } \\
\text { 1993USGS CRM-1 }\end{array}$ & CRM1 & M & 41.99694 & -91.681847 & 42.5 & $11 / 4 / 1993$ & $5 / 29 / 2003$ & $11 / 4 / 1993$ & $5 / 29 / 2003$ & No \\
\hline 415951091440501 & $\begin{array}{l}\text { 083N08W13CBCB } \\
\text { 1998USGS CRM-21 }\end{array}$ & CRM21 & M & 41.9975 & -91.734904 & 42.5 & $3 / 25 / 1998$ & $3 / 25 / 1998$ & $10 / 29 / 1998$ & 3/1/1999 & No \\
\hline 415953091435001 & $\begin{array}{l}\text { 083N08W13CBDA } \\
\text { 1993USGS CRM-3 }\end{array}$ & CRM3 & M & 41.99805 & -91.730737 & 39.5 & $3 / 25 / 1993$ & $9 / 15 / 2009$ & $11 / 4 / 1993$ & $8 / 4 / 2008$ & No \\
\hline 415953091435003 & $\begin{array}{l}\text { 083N08W13CBDA } \\
\text { 2009USGS CRM-3A }\end{array}$ & CRM3A & M & 41.99806 & -91.730556 & 42.5 & $3 / 8 / 2010$ & $8 / 19 / 2013$ & $6 / 6 / 2011$ & $3 / 11 / 2015$ & No \\
\hline 415953091435301 & $\begin{array}{l}\text { 083N08W13CBDA } \\
\text { 1993USGS CRM-4 }\end{array}$ & CRM4 & M & 41.99805 & -91.731571 & 42.5 & $3 / 25 / 1993$ & $3 / 10 / 2008$ & $11 / 1 / 1993$ & $3 / 10 / 2008$ & No \\
\hline 415953091435302 & $\begin{array}{l}\text { 083N08W13CBDA } \\
\text { 2009USGS CRM-4A }\end{array}$ & CRM4A & M & 41.99806 & -91.731389 & 42.5 & $3 / 8 / 2010$ & $6 / 4 / 2018$ & $7 / 20 / 2010$ & $10 / 30 / 2018$ & Yes \\
\hline 415954091440301 & $\begin{array}{l}\text { 083N08W13CBBC } \\
\text { 1997USGS CRM-20 }\end{array}$ & CRM20 & M & 41.99833 & -91.734349 & 42.5 & $11 / 6 / 1997$ & $3 / 25 / 1998$ & $12 / 5 / 2012$ & $3 / 2 / 2020$ & Yes \\
\hline 420009091432101 & $\begin{array}{l}\text { 083N08W13ACAC } \\
\text { 1998USGS CRM-27 }\end{array}$ & CRM27 & M & 42.0025 & -91.722682 & 23.0 & $11 / 16 / 1998$ & $5 / 27 / 2003$ & $3 / 4 / 1998$ & $5 / 27 / 2003$ & No \\
\hline 420012091432601 & $\begin{array}{l}\text { 083N08W13ACBA } \\
\text { 1998USGS CRM-25 }\end{array}$ & CRM25 & M & 42.00333 & -91.72407 & 18.0 & $11 / 17 / 1998$ & $5 / 27 / 2003$ & 3/4/1998 & $5 / 27 / 2003$ & No \\
\hline 420013091431001 & $\begin{array}{l}\text { 083N08W13AACC } \\
\text { 1998USGS CRM-22 }\end{array}$ & CRM22 & M & 42.00361 & -91.719626 & 22.5 & $11 / 16 / 1998$ & $11 / 3 / 2010$ & $2 / 18 / 1998$ & 9/9/2009 & No \\
\hline 420013091442801 & $\begin{array}{l}\text { 083N08W14A 1996USGS } \\
\text { CRM-19 }\end{array}$ & CRM19 & M & 42.00361 & -91.741293 & 42.0 & $9 / 24 / 1996$ & 8/20/1998 & $1 / 22 / 1997$ & $6 / 13 / 2001$ & Yes \\
\hline 420014091431101 & $\begin{array}{l}\text { 083N08W13AACC } \\
\text { 1998USGS CRM-24 }\end{array}$ & CRM24 & M & 42.00389 & -91.719904 & 47.0 & $11 / 16 / 1998$ & $2 / 27 / 2001$ & 3/4/1998 & $6 / 29 / 2001$ & No \\
\hline 420014091431201 & $\begin{array}{l}\text { 083N08W13AAC } \\
\text { 2005USGS CRM-30 }\end{array}$ & CRM30 & M & 42.00392 & -91.719889 & 42.0 & $2 / 28 / 2006$ & $6 / 27 / 2006$ & -- & -- & No \\
\hline 420014091442701 & $\begin{array}{l}\text { 083N08W14A 1996USGS } \\
\text { CRM-18 }\end{array}$ & CRM18 & M & 42.00389 & -91.741015 & 42.5 & $9 / 24 / 1996$ & $8 / 20 / 1998$ & $1 / 22 / 1997$ & $3 / 2 / 2020$ & Yes \\
\hline 420015091431201 & $\begin{array}{l}\text { 083N08W13AAC } \\
\text { 2005USGS CRM-29 }\end{array}$ & CRM29 & M & 42.00403 & -91.719861 & 22.0 & $2 / 28 / 2006$ & $5 / 17 / 2007$ & -- & -- & No \\
\hline
\end{tabular}


Table 1. Wells monitored for water levels and water quality, Linn County, lowa, 1990-2019.-Continued

[See Meppelink and Kalkhoff (2021) for the complete list of wells monitored during this study; only wells mentioned in this report are listed here. Dates are given in month/day/year. USGS, U.S. Geological Survey; ft, foot; M, monitoring; RP, Ranney production; VP, vertical production; --, not applicable]

\begin{tabular}{|c|c|c|c|c|c|c|c|c|c|c|c|}
\hline \multirow{2}{*}{ USGS site number ${ }^{1}$} & \multirow{2}{*}{ USGS site name ${ }^{1}$} & \multirow{2}{*}{$\begin{array}{c}\text { Site short } \\
\text { name (fig. 5) }\end{array}$} & \multirow{2}{*}{$\begin{array}{l}\text { Well } \\
\text { type }\end{array}$} & \multirow{2}{*}{ Latitude $^{1}$} & \multirow{2}{*}{ Longitude $^{1}$} & \multirow{2}{*}{$\begin{array}{l}\text { Well } \\
\text { depth' } \\
\text { (ft) }\end{array}$} & \multicolumn{2}{|c|}{$\begin{array}{l}\text { Period of water quality } \\
\text { record }^{1}\end{array}$} & \multicolumn{2}{|c|}{$\begin{array}{l}\text { Period of water level } \\
\text { record }{ }^{1}\end{array}$} & \multirow{2}{*}{$\begin{array}{l}\text { Continuous } \\
\text { water-level } \\
\text { data }\end{array}$} \\
\hline & & & & & & & $\begin{array}{c}\text { Beginning } \\
\text { date }\end{array}$ & $\begin{array}{c}\text { Ending } \\
\text { date }\end{array}$ & $\begin{array}{l}\text { Beginning } \\
\text { date }\end{array}$ & $\begin{array}{l}\text { Ending } \\
\text { date }\end{array}$ & \\
\hline \multicolumn{12}{|c|}{ Alluvial aquifer-Continued } \\
\hline 420015091431301 & $\begin{array}{l}\text { 083N08W13AACC } \\
\text { 1998USGS CRM-23 }\end{array}$ & CRM23 & M & 42.00417 & -91.720459 & 27.0 & $11 / 16 / 1998$ & $12 / 18 / 2006$ & $3 / 4 / 1998$ & $9 / 27 / 2004$ & No \\
\hline 420018091430801 & $\begin{array}{l}\text { 083N08W13AACA } \\
\text { 1998USGS CRM-26 }\end{array}$ & CRM26 & M & 42.005 & -91.71907 & 23.0 & $11 / 16 / 1998$ & $5 / 27 / 2003$ & 8/12/1998 & $12 / 4 / 2012$ & No \\
\hline 420019091442501 & $\begin{array}{l}\text { 083N08W14AAC } \\
\text { 1996USGS CRM-17 }\end{array}$ & CRM17 & M & 42.00528 & -91.74046 & 20.0 & $9 / 24 / 1996$ & 8/19/1998 & $7 / 25 / 1997$ & $3 / 2 / 2020$ & Yes \\
\hline 420019091442502 & $\begin{array}{l}\text { 083N08W14AAC } \\
\text { 1996USGS CRM-16 }\end{array}$ & CRM16 & M & 42.00528 & -91.74046 & 40.0 & 9/24/1996 & $8 / 19 / 1998$ & $1 / 23 / 1997$ & $3 / 2 / 2020$ & Yes \\
\hline 420030091424601 & $\begin{array}{l}\text { 083N07W07CCCB } \\
\text { 1994USGS CRM-10 }\end{array}$ & CRM10 & M & 42.00833 & -91.712959 & 38.5 & 2/11/1994 & $6 / 18 / 1996$ & $3 / 2 / 1994$ & $12 / 4 / 2012$ & No \\
\hline 420031091441801 & $\begin{array}{l}\text { 083N08W11DDC } \\
\text { 1996USGS CRM-15 }\end{array}$ & CRM15 & M & 42.00861 & -91.738515 & 18.0 & 9/24/1996 & $8 / 19 / 1998$ & $1 / 31 / 1997$ & $3 / 2 / 2020$ & Yes \\
\hline 420031091441802 & $\begin{array}{l}\text { 083N08W11DDC } \\
\text { 1996USGS CRM-14 }\end{array}$ & CRM14 & M & 42.00861 & -91.738515 & 27.5 & $5 / 29 / 1997$ & $5 / 29 / 1997$ & 10/29/1998 & $3 / 2 / 2020$ & No \\
\hline 420032091424901 & $\begin{array}{l}\text { 083N07W07CCDB } \\
\text { 1994USGS CRM-9 }\end{array}$ & CRM9 & M & 42.00889 & -91.713792 & 37.5 & $3 / 2 / 1994$ & 8/27/1996 & $6 / 15 / 1994$ & $6 / 29 / 2001$ & No \\
\hline 420033091420301 & $\begin{array}{l}\text { 083N07W07DDBD } \\
\text { 1993USGS CRM-2 }\end{array}$ & CRM2 & M & 42.00917 & -91.701014 & 42.5 & $11 / 4 / 1993$ & $5 / 28 / 2003$ & $11 / 4 / 1993$ & $2 / 18 / 2003$ & Yes \\
\hline 420408091470201 & $\begin{array}{l}\text { 084N08W21DCBB } \\
\text { 1993USGS CRM-5 }\end{array}$ & CRM5 & M & 42.06889 & -91.784069 & 29.5 & $3 / 8 / 1993$ & $11 / 4 / 1993$ & $3 / 8 / 1993$ & $12 / 1 / 1994$ & No \\
\hline 415952091440400 & $\begin{array}{l}\text { 083N08W13CBCB } \\
\text { 1994Cedar Rapids } \\
\text { Ranney } 1\end{array}$ & Ran1 & MR & 41.99778 & -91.734626 & 59.5 & $10 / 8 / 2003$ & $12 / 5 / 2019$ & -- & -- & No \\
\hline 420004091442300 & $\begin{array}{l}\text { 083N08W14ADCC } \\
\text { 1995Cedar Rapids } \\
\text { Ranney } 2\end{array}$ & $\operatorname{Ran} 2$ & MR & 42.00111 & -91.739904 & 49.6 & $3 / 24 / 2004$ & $4 / 16 / 2019$ & -- & -- & No \\
\hline 420010091431801 & $\begin{array}{l}\text { 083N08W13ACAD } 56471 \\
\text { 2002Cedar Rapids Smnl } \\
\text { Ranney } 4\end{array}$ & Ran4 & MR & 42.00278 & -91.721667 & 60.4 & $5 / 29 / 2003$ & $12 / 5 / 2019$ & -- & -- & No \\
\hline 420035091422301 & $\begin{array}{l}\text { 083N07W07DCBC } 56470 \\
\text { 2002Cedar Rapids West } \\
\text { Ranney } 3\end{array}$ & Ran3 & MR & 42.00972 & -91.706389 & 57.0 & $5 / 29 / 2003$ & $12 / 5 / 2019$ & -- & -- & Yes \\
\hline
\end{tabular}


[See Meppelink and Kalkhoff (2021) for the complete list of wells monitored during this study; only wells mentioned in this report are listed here. Dates are given in month/day/year. USGS, U.S. Geological Survey; ft, foot; M, monitoring; RP, Ranney production; VP, vertical production; --, not applicable]

\begin{tabular}{|c|c|c|c|c|c|c|c|c|c|c|c|}
\hline \multirow{2}{*}{ USGS site number ${ }^{1}$} & \multirow{2}{*}{ USGS site name $^{1}$} & \multirow{2}{*}{$\begin{array}{c}\text { Site short } \\
\text { name (fig. 5) }\end{array}$} & \multirow{2}{*}{$\begin{array}{l}\text { Well } \\
\text { type }\end{array}$} & \multirow{2}{*}{ Latitude $^{1}$} & \multirow{2}{*}{ Longitude $^{1}$} & \multirow{2}{*}{$\begin{array}{c}\text { Well } \\
\text { depth' } \\
\text { (ft) }\end{array}$} & \multicolumn{2}{|c|}{$\begin{array}{l}\text { Period of water quality } \\
\text { record }^{1}\end{array}$} & \multicolumn{2}{|c|}{$\begin{array}{l}\text { Period of water level } \\
\text { record }{ }^{1}\end{array}$} & \multirow{2}{*}{$\begin{array}{l}\text { Continuous } \\
\text { water-leve } \\
\text { data }\end{array}$} \\
\hline & & & & & & & $\begin{array}{l}\text { Beginning } \\
\text { date }\end{array}$ & $\begin{array}{l}\text { Ending } \\
\text { date }\end{array}$ & $\begin{array}{l}\text { Beginning } \\
\text { date }\end{array}$ & $\begin{array}{l}\text { Ending } \\
\text { date }\end{array}$ & \\
\hline \multicolumn{12}{|c|}{ Alluvial aquifer-Continued } \\
\hline 420036091444001 & $\begin{array}{l}\text { 083N08W11DCBC } \\
\text { 2013Cedar Rapids } \\
\text { Ranney } 6\end{array}$ & Ran6 & MR & 42.01 & -91.744611 & 74.3 & $3 / 12 / 2014$ & $12 / 5 / 2019$ & -- & -- & No \\
\hline 415949091404901 & $\begin{array}{l}\text { 083N07W17DACD } 37819 \\
\text { 1961Cedar Rapids } \\
\text { East } 3\end{array}$ & East3 & MV & 41.99694 & -91.680458 & 72.0 & $6 / 29 / 2004$ & $6 / 29 / 2004$ & $11 / 1 / 1993$ & $11 / 1 / 1993$ & No \\
\hline 415949091405201 & $\begin{array}{l}\text { 083N07W17DACC } 26138 \\
\text { 1980Cedar Rapids East } \\
10\end{array}$ & East10 & MV & 41.99694 & -91.681847 & 67.0 & $11 / 2 / 1993$ & $11 / 2 / 1993$ & $11 / 1 / 1993$ & $11 / 1 / 1993$ & No \\
\hline 415952091405701 & $\begin{array}{l}\text { 083N07W17DBDA } 27724 \\
\text { 1984Cedar Rapids East } \\
12\end{array}$ & East12 & MV & 41.99722 & -91.682681 & 61.0 & $11 / 2 / 1993$ & $11 / 2 / 1993$ & $11 / 1 / 1993$ & $11 / 1 / 1993$ & No \\
\hline 415952091434301 & $\begin{array}{l}\text { 083N08W13CACA } 37642 \\
\text { 1971Cedar Rapids } \\
\text { Seminole } 9\end{array}$ & Sem9 & MV & 41.99778 & -91.728793 & 57.4 & $2 / 20 / 1986$ & $8 / 21 / 2006$ & $11 / 1 / 1993$ & $11 / 1 / 1993$ & No \\
\hline 415953091435201 & $\begin{array}{c}\text { 083N08W13CBDA } 37817 \\
\text { 1971Cedar Rapids } 10\end{array}$ & Sem10 & MV & 41.99805 & -91.731293 & 68.6 & $3 / 25 / 1993$ & 2/15/1996 & -- & -- & No \\
\hline 415955091433601 & 083N08W13CAAC 1970 & -- & MV & 41.99861 & -91.727404 & 57.3 & $11 / 2 / 1993$ & $11 / 2 / 1993$ & 11/1/1993 & $11 / 1 / 1993$ & No \\
\hline 415959091410501 & $\begin{array}{l}\text { 083N07W17DBAB } 28333 \\
\text { 1984Cedar Rapids East } \\
14\end{array}$ & East14 & MV & 41.99833 & -91.683792 & 64.5 & 8/29/1991 & 8/29/1991 & 11/1/1993 & $11 / 1 / 1993$ & No \\
\hline 415959091433001 & $\begin{array}{l}\text { 083N08W13DBBB } 37815 \\
\text { 1971Cedar Rapids } \\
\text { Seminole } 7\end{array}$ & Sem7 & MV & 41.99972 & -91.725459 & 63.5 & 8/29/1991 & 8/29/1991 & 11/1/1993 & 11/1/1993 & No \\
\hline 420006091432201 & $\begin{array}{l}\text { 083N08W13ACDB } 37641 \\
\text { 1971Cedar Rapids } \\
\text { Seminole } 6\end{array}$ & Sem6 & MV & 42.00167 & -91.722959 & 61.1 & 8/26/1992 & $4 / 13 / 2016$ & $11 / 1 / 1993$ & $11 / 1 / 1993$ & No \\
\hline 420012091412801 & 083N07W17BDBB & -- & MV & 42.00333 & -91.691292 & 66.0 & $11 / 2 / 1993$ & $11 / 2 / 1993$ & 11/1/1993 & 11/1/1993 & No \\
\hline 420013091442000 & $\begin{array}{l}\text { 083N08W14ADBB } 43186 \\
\text { 1991Cedar Rapids } \\
\text { Seminole } 17\end{array}$ & Sem17 & MV & 42.00361 & -91.739071 & 54.0 & $10 / 7 / 2003$ & $12 / 5 / 2019$ & 11/1/1993 & $11 / 1 / 1993$ & No \\
\hline
\end{tabular}


Table 1. Wells monitored for water levels and water quality, Linn County, lowa, 1990-2019.-Continued

[See Meppelink and Kalkhoff (2021) for the complete list of wells monitored during this study; only wells mentioned in this report are listed here. Dates are given in month/day/year. USGS, U.S. Geological Survey; ft, foot; M, monitoring; RP, Ranney production; VP, vertical production; --, not applicable]

\begin{tabular}{|c|c|c|c|c|c|c|c|c|c|c|c|}
\hline \multirow{2}{*}{ USGS site number ${ }^{1}$} & \multirow{2}{*}{ USGS site name ${ }^{1}$} & \multirow{2}{*}{$\begin{array}{c}\text { Site short } \\
\text { name (fig. 5) }\end{array}$} & \multirow{2}{*}{$\begin{array}{l}\text { Well } \\
\text { type }\end{array}$} & \multirow{2}{*}{ Latitude ${ }^{1}$} & \multirow{2}{*}{ Longitude ${ }^{1}$} & \multirow{2}{*}{$\begin{array}{c}\text { Well } \\
\text { depth' } \\
\text { (ft) }\end{array}$} & \multicolumn{2}{|c|}{$\begin{array}{l}\text { Period of water quality } \\
\text { record }{ }^{1}\end{array}$} & \multicolumn{2}{|c|}{$\begin{array}{l}\text { Period of water level } \\
\text { record }^{1}\end{array}$} & \multirow{2}{*}{$\begin{array}{l}\text { Continuous } \\
\text { water-level } \\
\text { data }\end{array}$} \\
\hline & & & & & & & $\begin{array}{l}\text { Beginning } \\
\text { date }\end{array}$ & $\begin{array}{l}\text { Ending } \\
\text { date }\end{array}$ & $\begin{array}{l}\text { Beginning } \\
\text { date }\end{array}$ & $\begin{array}{l}\text { Ending } \\
\text { date }\end{array}$ & \\
\hline \multicolumn{12}{|c|}{ Alluvial aquifer-Continued } \\
\hline 420013091442501 & $\begin{array}{l}\text { 083N08W14ACAA } 43187 \\
\text { 1991Cedar Rapids } \\
\text { Seminole } 18\end{array}$ & Sem18 & MV & 42.00361 & -91.74046 & 52.0 & $4 / 1 / 2005$ & $6 / 28 / 2016$ & $11 / 1 / 1993$ & $11 / 1 / 1993$ & No \\
\hline 420015091430601 & $\begin{array}{l}\text { 083N08W13AACC } 37639 \\
\text { 1971Cedar Rapids } \\
\text { Seminole } 4\end{array}$ & Sem4 & MV & 42.00417 & -91.718515 & 55.4 & $11 / 1 / 1993$ & $8 / 21 / 2006$ & $11 / 1 / 1993$ & $11 / 1 / 1993$ & No \\
\hline 420017091413401 & $\begin{array}{l}\text { 083N07W17BBDA } 37631 \\
\text { 1964Cedar Rapids } \\
\text { West } 2\end{array}$ & West2 & MV & 42.00472 & -91.692959 & 72.0 & $11 / 2 / 1993$ & $11 / 2 / 1993$ & $11 / 1 / 1993$ & $11 / 1 / 1993$ & No \\
\hline 420020091430601 & $\begin{array}{l}\text { 083N08W13AACA } \\
\text { 1971Cedar Rapids } \\
\text { Seminole } 3\end{array}$ & Sem3 & MV & 42.00555 & -91.718515 & 62.8 & $11 / 1 / 1993$ & $11 / 1 / 1993$ & $11 / 1 / 1993$ & $11 / 1 / 1993$ & No \\
\hline 420020091442501 & $\begin{array}{l}\text { 083N08W14ACDA } 37830 \\
\text { 1990Cedar Rapids } \\
\text { Seminole } 14\end{array}$ & Sem14 & MV & 42.00555 & -91.74046 & 59.0 & $11 / 2 / 1993$ & $9 / 27 / 2004$ & $11 / 1 / 1993$ & $11 / 1 / 1993$ & No \\
\hline 420024091414601 & $\begin{array}{l}\text { 083N07W17BBBB } 37632 \\
\text { 1964Cedar Rapids } \\
\text { West } 3\end{array}$ & West3 & MV & 42.00667 & -91.696292 & 72.0 & $8 / 20 / 1987$ & $11 / 2 / 1993$ & $11 / 1 / 1993$ & $11 / 1 / 1993$ & No \\
\hline 420025091425801 & $\begin{array}{l}\text { 083N08W13AAAA } 37814 \\
\text { 1971Cedar Rapids } \\
\text { Seminole } 2\end{array}$ & Sem2 & MV & 42.00694 & -91.716292 & 53.9 & $11 / 1 / 1993$ & 2/15/1996 & $11 / 1 / 1993$ & $11 / 1 / 1993$ & No \\
\hline 420029091443001 & $\begin{array}{l}\text { 083N08W11DCDB } 37832 \\
\text { 1990Cedar Rapids } \\
\text { Seminole } 16\end{array}$ & Sem16 & MV & 42.00805 & -91.741849 & 65.0 & $11 / 2 / 1993$ & $11 / 2 / 1993$ & $11 / 1 / 1993$ & $11 / 1 / 1993$ & No \\
\hline 420030091424901 & $\begin{array}{l}\text { 083N07W07CCD } 37813 \\
\text { 1971Cedar Rapids } \\
\text { Seminole } 1\end{array}$ & Sem 1 & MV & 42.00889 & -91.712681 & 63.9 & $10 / 25 / 1993$ & $8 / 27 / 1996$ & -- & -- & No \\
\hline 420031091415701 & $\begin{array}{l}\text { 083N07W07DDDB } 26136 \\
\text { 1980Cedar Rapids } \\
\text { West } 9\end{array}$ & West9 & MV & 42.00861 & -91.699347 & 63.0 & $8 / 29 / 1991$ & $8 / 29 / 1991$ & $11 / 1 / 1993$ & $11 / 1 / 1993$ & No \\
\hline 420034091444101 & $\begin{array}{l}\text { 083N08W11DCBC } 43192 \\
\text { 1991Cedar Rapids } \\
\text { Seminole } 23\end{array}$ & Sem 23 & MV & 42.00944 & -91.744349 & 57.0 & $6 / 27 / 2006$ & $8 / 21 / 2006$ & $11 / 1 / 1993$ & $11 / 1 / 1993$ & No \\
\hline
\end{tabular}


[See Meppelink and Kalkhoff (2021) for the complete list of wells monitored during this study; only wells mentioned in this report are listed here. Dates are given in month/day/year. USGS, U.S. Geological Survey; ft, foot; M, monitoring; RP, Ranney production; VP, vertical production; --, not applicable]

\begin{tabular}{|c|c|c|c|c|c|c|c|c|c|c|c|}
\hline \multirow{2}{*}{ USGS site number ${ }^{1}$} & \multirow{2}{*}{ USGS site name ${ }^{1}$} & \multirow{2}{*}{$\begin{array}{c}\text { Site short } \\
\text { name (fig. 5) }\end{array}$} & \multirow{2}{*}{$\begin{array}{l}\text { Well } \\
\text { type }\end{array}$} & \multirow{2}{*}{ Latitude $^{1}$} & \multirow{2}{*}{ Longitude $^{1}$} & \multirow{2}{*}{$\begin{array}{c}\text { Well } \\
\text { depth' } \\
\text { (ft) }\end{array}$} & \multicolumn{2}{|c|}{$\begin{array}{l}\text { Period of water quality } \\
\text { record }^{1}\end{array}$} & \multicolumn{2}{|c|}{$\begin{array}{c}\text { Period of water level } \\
\text { record }^{1}\end{array}$} & \multirow{2}{*}{$\begin{array}{l}\text { Continuous } \\
\text { water-level } \\
\text { data }\end{array}$} \\
\hline & & & & & & & $\begin{array}{l}\text { Beginning } \\
\text { date }\end{array}$ & $\begin{array}{l}\text { Ending } \\
\text { date }\end{array}$ & $\begin{array}{l}\text { Beginning } \\
\text { date }\end{array}$ & $\begin{array}{c}\text { Ending } \\
\text { date }\end{array}$ & \\
\hline \multicolumn{12}{|c|}{ Alluvial aquifer-Continued } \\
\hline 420036091420901 & $\begin{array}{l}\text { 083N07W07DCAA } 26137 \\
\text { 1980Cedar Rapids West } \\
10\end{array}$ & West10 & MV & 42.01 & -91.702681 & 67.0 & $11 / 3 / 1993$ & $11 / 3 / 1993$ & $11 / 1 / 1993$ & $11 / 1 / 1993$ & No \\
\hline 420036091422701 & $\begin{array}{l}\text { 083N07W07CDAA } 37636 \\
\text { 1964Cedar Rapids } \\
\text { West } 7\end{array}$ & West7 & MV & 42.01 & -91.707681 & 70.0 & $11 / 3 / 1993$ & $11 / 3 / 1993$ & $11 / 1 / 1993$ & $11 / 1 / 1993$ & No \\
\hline \multicolumn{12}{|c|}{ Devonian aquifer } \\
\hline 415949091405402 & $\begin{array}{l}\text { 083N07W17DAC } \\
\text { 1993USGS CRM-12 }\end{array}$ & CRM12 & M & 41.99694 & -91.681847 & 75 & $5 / 15 / 1995$ & $8 / 20 / 1997$ & $12 / 1 / 1994$ & $3 / 2 / 2020$ & Yes \\
\hline 415954091435301 & $\begin{array}{l}\text { 083N08W13ADBC } \\
\text { 1993USGS CRM-6 }\end{array}$ & CRM6 & M & 41.99833 & -91.731571 & 94 & $11 / 3 / 1993$ & $6 / 18 / 1996$ & $11 / 3 / 1993$ & $6 / 29 / 2001$ & No \\
\hline 415954091435302 & $\begin{array}{l}\text { 083N08W13ADBC } \\
\text { 2010USGS CRM-6A }\end{array}$ & CRM6A & M & 41.99833 & -91.731389 & 97 & $6 / 6 / 2011$ & $11 / 5 / 2018$ & $6 / 6 / 2011$ & $10 / 30 / 2018$ & Yes \\
\hline 420032091424701 & $\begin{array}{l}\text { 083N07W07CCDB } \\
\text { 1994USGS CRM-7 }\end{array}$ & CRM7 & M & 42.00889 & -91.713237 & 84 & $12 / 21 / 1993$ & $8 / 27 / 1996$ & $6 / 15 / 1994$ & $6 / 29 / 2001$ & No \\
\hline 420033091420302 & $\begin{array}{l}\text { 083N07W07DDBD } \\
\text { 1993USGS CRM-11 }\end{array}$ & CRM11 & M & 42.00917 & -91.701014 & 85 & $10 / 19 / 1995$ & 8/20/1997 & $12 / 1 / 1994$ & $5 / 22 / 2019$ & Yes \\
\hline
\end{tabular}

${ }^{1}$ These data are from the USGS National Water Information System (NWIS) database (USGS, 2020). Search for site information using the site number. 
Table 2. Sites on the Cedar River monitored for stage, flow, and water quality, Linn County, lowa, 1990-2019.

[Dates are given in month/day/year. USGS, U.S. Geological Survey; $\mathrm{mi}^{2}$, square mile; Present, date of publication; --, not applicable]

\begin{tabular}{|c|c|c|c|c|c|c|c|c|c|c|c|}
\hline \multirow{2}{*}{ USGS site number ${ }^{1}$} & \multirow{2}{*}{ USGS site name ${ }^{1}$} & \multirow{2}{*}{$\begin{array}{c}\text { Site short } \\
\text { name } \\
\text { (fig. 1) }\end{array}$} & \multirow{2}{*}{ Latitude $^{1}$} & \multirow{2}{*}{ Longitude $^{1}$} & \multirow{2}{*}{$\begin{array}{l}\text { Drainage } \\
\text { area }^{1} \\
\left(\mathrm{mi}^{2}\right)\end{array}$} & \multicolumn{2}{|c|}{$\begin{array}{l}\text { Period of water quality } \\
\text { record }{ }^{1}\end{array}$} & \multirow{2}{*}{$\begin{array}{c}\text { Real- } \\
\text { time } \\
\text { water } \\
\text { quality }\end{array}$} & \multicolumn{2}{|c|}{$\begin{array}{l}\text { Period of streamflow } \\
\text { record }{ }^{1}\end{array}$} & \multirow{2}{*}{$\begin{array}{c}\text { Real- } \\
\text { time } \\
\text { water } \\
\text { level }\end{array}$} \\
\hline & & & & & & $\begin{array}{c}\text { Beginning } \\
\text { date }\end{array}$ & $\begin{array}{l}\text { Ending } \\
\text { date }\end{array}$ & & $\begin{array}{l}\text { Beginning } \\
\text { date }\end{array}$ & $\begin{array}{l}\text { Ending } \\
\text { date }\end{array}$ & \\
\hline 05464420 & $\begin{array}{l}\text { Cedar River at Blairs } \\
\text { Ferry Road at Palo, } \\
\text { Iowa }\end{array}$ & SW-1 & 42.06916 & -91.78518 & 6,342 & $10 / 28 / 1992$ & Present & Yes & $10 / 20 / 2017$ & Present & Yes \\
\hline 415953091435300 & $\begin{array}{l}\text { 083N08W13CBDA } \\
\text { USGS CRM-SW-2 }\end{array}$ & SW-2 & 41.99805 & -91.73157 & 6,480 & -- & -- & No & -- & -- & No \\
\hline 05464480 & $\begin{array}{l}\text { Cedar River at } \\
\text { Edgewood Road at } \\
\text { Cedar Rapids, Iowa }\end{array}$ & SW-3 & 42.01167 & -91.7049 & 6,489 & $11 / 23 / 1992$ & $8 / 17 / 2016$ & No & $3 / 25 / 2009$ & Present & Yes \\
\hline 05464500 & $\begin{array}{l}\text { Cedar River at Cedar } \\
\text { Rapids, Iowa }\end{array}$ & SW-4 & 41.97195 & -91.66712 & 6,510 & 9/6/1906 & $6 / 14 / 2011$ & Yes & $10 / 1 / 1902$ & Present & Yes \\
\hline
\end{tabular}

${ }^{1}$ These data are from the USGS National Water Information System (NWIS) database (USGS, 2020). Search for site information using the site number. 
alluvial aquifer (Boyd, 1999). Several monitoring wells were screened in the underlying Devonian aquifer (table 1) to help understand how mixing of water from the bedrock and alluvial aquifers affects water supplying residential and industrial purposes. Monitoring wells provide data on small, localized areas of the aquifer because of their short-screened interval (generally $10 \mathrm{ft}$ ) and low pumping rate for sampling. Vertical production wells provide an integrated sample through the entire depth of the aquifer. Ranney production wells provide data from a larger area than the other well types because of their high pumping rate and because screens radiate horizontally several hundred feet from the central caisson.

Water in the Cedar River naturally moves into parts of the alluvial aquifer during high-flow conditions (Squillace, 1996; Squillace and others, 1996) and is induced into the aquifer by pumping of large-capacity production wells (Boyd, 1998). Initially (from 1993 through 2016), the water quality of the Cedar River was characterized by samples collected at SW-3 (fig. 1). Sampling was moved about 10 miles upstream to SW-1 in September 2016 to better document the quality of Cedar River water flowing into Cedar Rapids.

High-frequency data measured by multiprobe instruments provided information on water-quality constituents (specific conductance, $\mathrm{pH}$, water temperature, and dissolved oxygen) in selected production and monitoring wells and the Cedar River at two intensive study sites in the Seminole well field from 1992 to 1997 (Schulmeyer and Schnoebelen, 1998). Highfrequency hydrological and water-quality data were collected to understand the effect of large groundwater withdrawals on hydrological interaction between the alluvial aquifer and the Cedar River.

\section{Discrete Water Sample Collection and Analysis}

Water samples were collected for several constituents during the study period, but only anthropogenic and natural constituents of concern for a water supply are emphasized in this report. Nutrients and organic pesticide compounds (table 3) were studied because of the intensive agricultural land use upstream in the Cedar River Basin. The water chemistry of the alluvial aquifer and its source waters was characterized by measuring physical characteristics and analyzing major ions, dissolved solids, nutrients, selected trace elements, and pesticides (table 3). Twenty-five commonly used pesticides were analyzed at the USGS National Water-Quality Laboratory under schedule 1379 until 2016 when a new analytical schedule (2033) was used to analyze 99 compounds, including more parent, degradate, and intermediate compounds (table 4).

Production wells were sampled after they were pumping for more than 1 hour to obtain a representative sample from the aquifer. Samples from production wells were obtained from a spigot near the pump. Samples were collected from the monitoring wells after a minimum of three well volumes of water were pumped from the well with a submersible pump to ensure a representative sample. All samples were collected after field measurements of specific conductance, $\mathrm{pH}$, water temperature, and dissolved oxygen stabilized.

Samples from wells and the Cedar River were collected quarterly to document long-term trends and seasonal variability in water quality in the Cedar River alluvial aquifer and its source waters: the Cedar River and the Devonian aquifer. Initially, vertical production wells were sampled, but quarterly sampling was switched to Ranney production wells when they were built and put into production beginning in the early 2000s. Ranney production wells were prioritized for sampling because their large pumping capacity contributed a large amount of the total volume of water pumped for water supply purposes.

During the study period, samples were also collected on short-term timeframes (months to years) for additional intensive targeted studies. An initial synoptic study in November 1993 to document variability in the alluvial aquifer resulted in samples being collected from 64 production and monitoring wells from Palo, Iowa, downstream through the three well fields in Cedar Rapids (Schulmeyer and Schnoebelen, 1998). A combination of discreet water samples and continuous water-quality data were collected from the Cedar River and monitoring wells at intensive study sites near vertical production wells Sem 10 and Sem1 from 1992 to 1996 to determine the relation between the Cedar River alluvial aquifer, the Cedar River, and the Devonian aquifer in terms of water quality and flow (Schulmeyer and Schnoebelen, 1998). A short-term study evaluated common pesticides and pesticide degradates in the alluvial aquifer near Cedar Rapids after springtime application to upstream cropland (Boyd, 2000).

A description of the analytical methods for major ions, nutrients, trace elements, and pesticide compounds (tables 3 and 4), previously reported by Meppelink and others (2019), follows. Nutrient concentrations were analyzed by colorimetric methods (Patton and Kryskalla, 2011) for dissolved species and alkaline persulfate digestion (Patton and Kryskalla, 2003) for unfiltered samples. Analytical methods used for major ions are described by Fishman (1993). Inductively coupled plasmaatomic emission spectrometry was used to determine boron concentrations (Struzeski and others, 1996). The high-temperature combustion method was used to determine the total organic carbon concentration in samples (American Public Health Association, American Water Works Association, and Water Environment Federation, 2017).

Pesticide samples collected from 1990 to 2016 were analyzed for 25 compounds including selected degradates and intermediates (USGS schedule 1379) with solid-phase extraction and capillary-column gas chromatography/mass spectrometry methods (Sandstrom and others, 1992). A newer $\mathrm{C}_{18}$ solid-phase extraction and gas chromatography/mass spectrometry method (Sandstrom and others, 2001) that resulted in lower reporting limits for more than 80 compounds (USGS schedule 2033) was used for analysis of pesticide samples beginning in 2017. 
Table 3. Carbon, nutrients, major ions, dissolved solids, and physical characteristics analyzed for in water-quality samples, Linn County, lowa, 1990-2019.

[Table modified from Meppelink and others (2019). USGS, U.S. Geological Survey; CASRN, Chemical Abstracts Service Registry Number; LRL, laboratory reporting level; --, not applicable; $\mathrm{mg} / \mathrm{L}$, milligram per liter; $\mathrm{NH}_{4}^{+}$, ammonium; $\mathrm{N}$, nitrogen; $\mathrm{P}$, phosphorus; $\mathrm{CaCO}_{3}$, calcium carbonate; $\mu \mathrm{g} / \mathrm{L}$, microgram per liter; $\mathrm{SiO}_{2}$, silicon dioxide; $\mu \mathrm{S} / \mathrm{cm}$ at $25{ }^{\circ} \mathrm{C}$, microsiemens per centimeter at 25 degrees Celsius; ${ }^{\circ} \mathrm{C}$, degree Celsius]

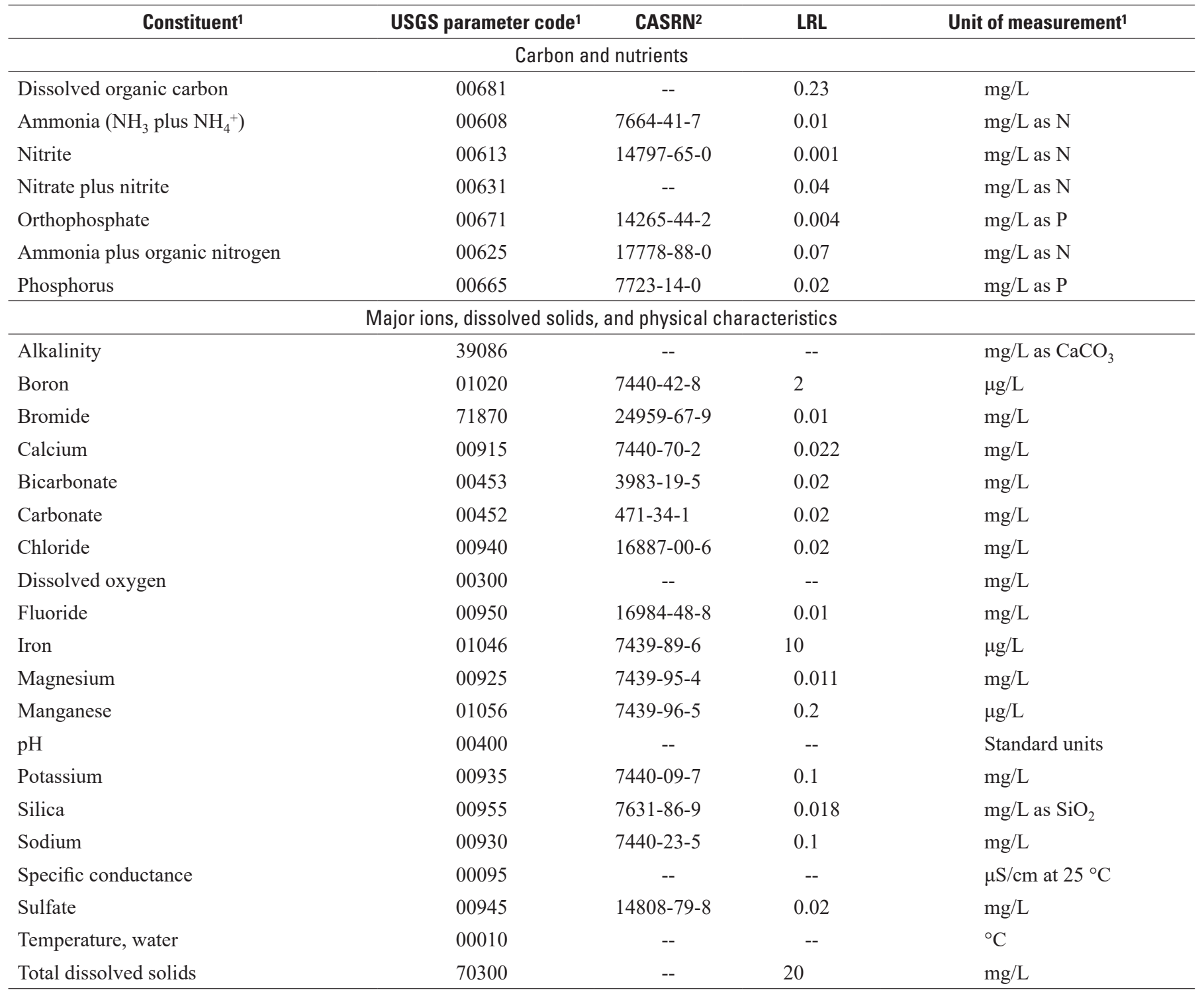

${ }^{1}$ These data are from the USGS National Water Information System (NWIS) database (USGS, 2020). Search using the parameter code to see full parameter names, descriptions, and other data in the database.

${ }^{2}$ This report contains CASRNs, which are a registered trademark of the American Chemical Society. The Chemical Abstracts Service recommends verifying the CASRNs through Chemical Abstracts Service Client Services. 
Table 4. Selected pesticides, degradates, and intermediates analyzed for in water-quality samples, Cedar Rapids, Linn County, lowa, 1990-2019.

[Table modified from Meppelink and others (2019). USGS, U.S. Geological Survey; CASRN, Chemical Abstracts Service Registry Number; LRL, laboratory reporting level; $\mu \mathrm{g} / \mathrm{L}$, microgram per liter; --, not analyzed; DCPA, dimethyl tetrachloroterephthalate; EPTC, S-ethyl dipropylthiocarbamate]

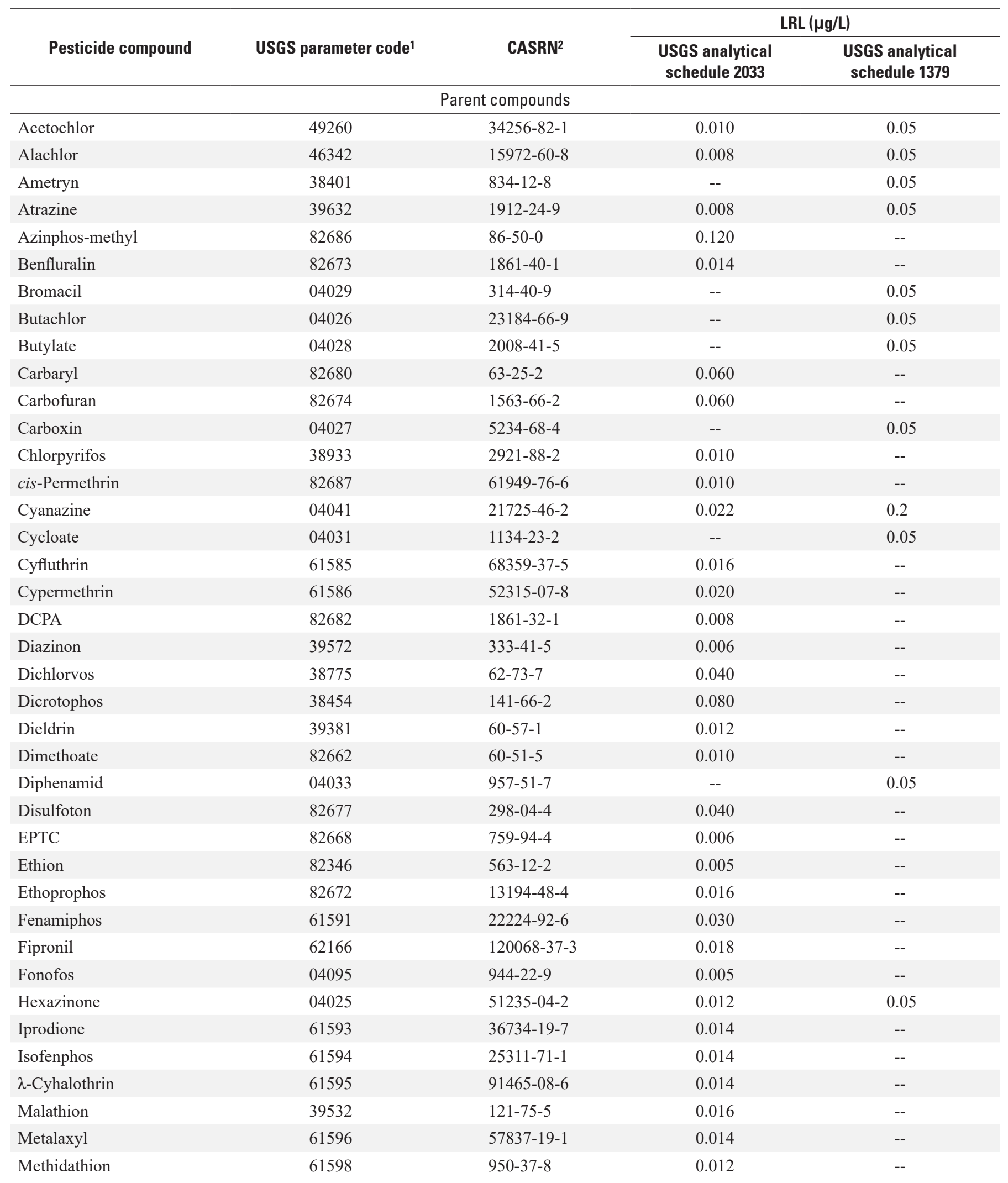


Table 4. Selected pesticides, degradates, and intermediates analyzed for in water-quality samples, Cedar Rapids, Linn County, lowa, 1990-2019.-Continued

[Table modified from Meppelink and others (2019). USGS, U.S. Geological Survey; CASRN, Chemical Abstracts Service Registry Number; LRL, laboratory reporting level; $\mu \mathrm{g} / \mathrm{L}$, microgram per liter; --, not analyzed; DCPA, dimethyl tetrachloroterephthalate; EPTC, S-ethyl dipropylthiocarbamate]

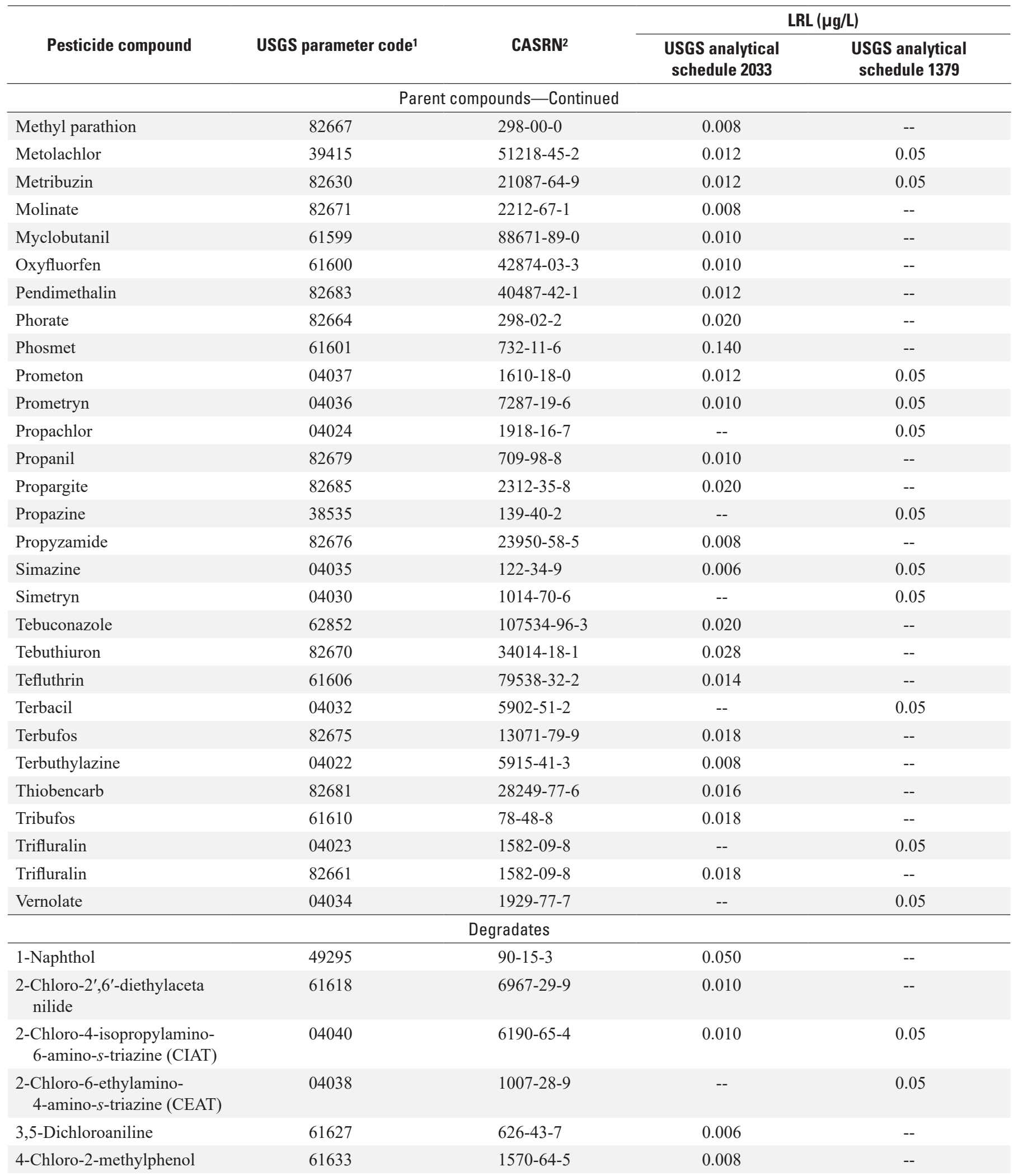


Table 4. Selected pesticides, degradates, and intermediates analyzed for in water-quality samples, Cedar Rapids, Linn County, lowa, 1990-2019.-Continued

[Table modified from Meppelink and others (2019). USGS, U.S. Geological Survey; CASRN, Chemical Abstracts Service Registry Number; LRL, laboratory reporting level; $\mu \mathrm{g} / \mathrm{L}$, microgram per liter; --, not analyzed; DCPA, dimethyl tetrachloroterephthalate; EPTC, S-ethyl dipropylthiocarbamate]

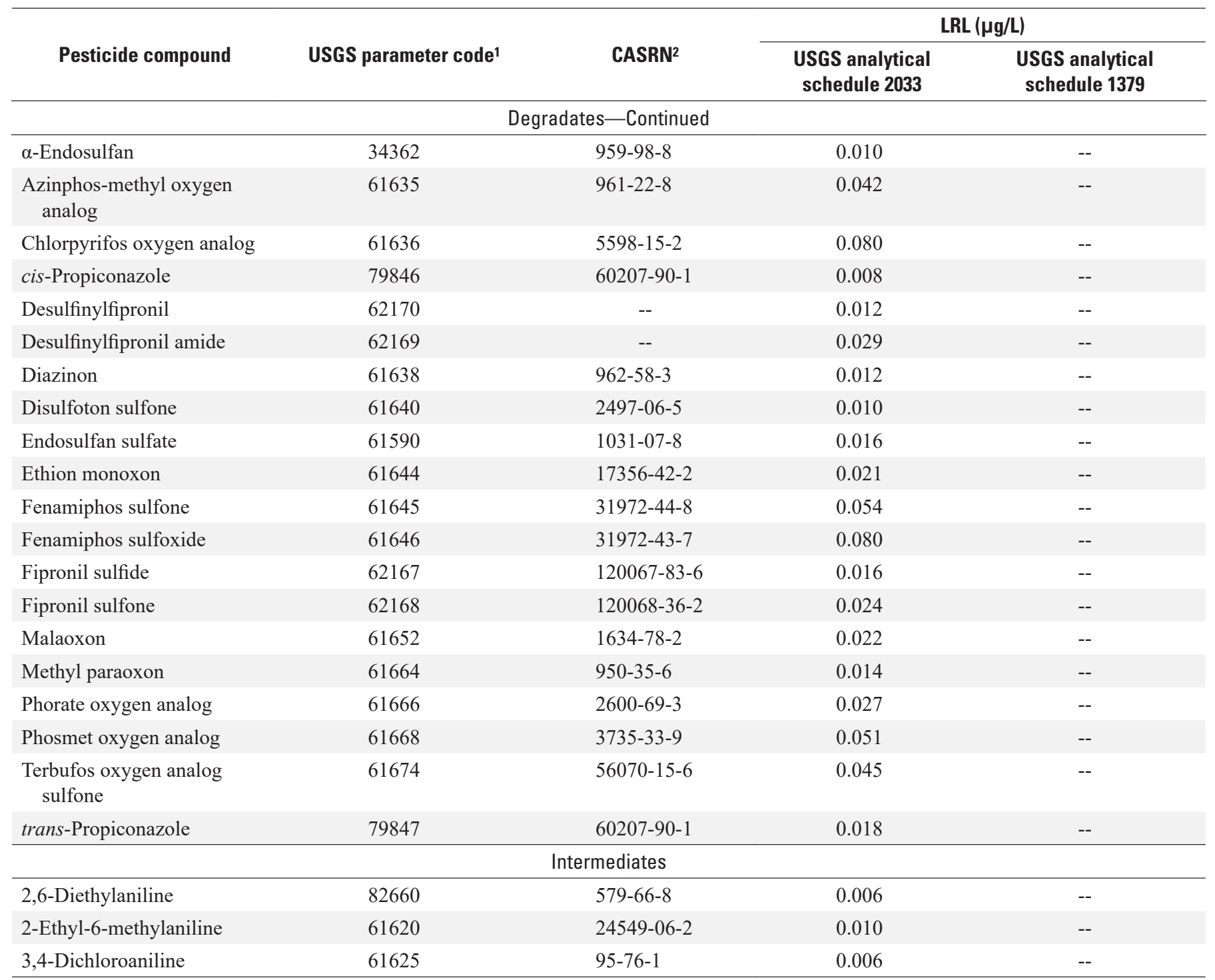

${ }^{1}$ These data are from the USGS National Water Information System (NWIS) database (USGS, 2020). Search using the parameter code to see full parameter names, descriptions, and other data in the database.

${ }^{2}$ This report contains CASRNs, which are a registered trademark of the American Chemical Society. The Chemical Abstracts Service recommends verifying the CASRNs through Chemical Abstracts Service Client Services. 


\section{High-Frequency Water-Quality Data Collection}

In addition to the discrete samples collected during the study, high-frequency water-quality data were collected to understand the rapid response to changes in the alluvial aquifer caused by rainfall, large-capacity production well pumping, and changing flow in the Cedar River. Multiparameter sondes were used to continually monitor water levels, specific conductance, $\mathrm{pH}$, water temperature, and dissolved-oxygen concentrations in selected wells at two intensive study sites and in the Cedar River (Schnoebelen and Schulmeyer, 1996). The multiprobe for the Cedar River (site SW-2) was installed in a 6-in. diameter pipe placed in the river. The multiparameter sondes recorded data internally at $15-, 30-$, or 60 -minute intervals that were generally retrieved every 4 weeks. The sonde sensors were cleaned and recalibrated using known specific conductance and $\mathrm{pH}$ standards after each data retrieval (Schnoebelen and Schulmeyer, 1996). Multiparameter sondes were installed in additional monitoring wells in 1998 to understand rapid water-quality changes over a large area of the Cedar Rapids well field (Boyd and others, 1999).

With the advent of new technology that allowed for instream measurement, nitrate- $\mathrm{N}$ concentrations were measured on 15-minute intervals in the Cedar River at Cedar Rapids beginning in 2009. Nitrate-N concentrations in the Cedar River were measured during the ice-free season (March or April through November) using an instream optical nitrate sensor (Pellerin and others, 2013). The nitrate sensor (Hach NITRATAX plus sc, Hach Company, Loveland, Colorado) initially was installed in the Cedar River at Cedar Rapids, Iowa (SW-4, fig. 1) and was operated at this site until October 2012, when the sensor was moved upstream about 14 miles to the Cedar River at Blairs Ferry Road at Palo, Iowa (SW-1). Nitrate-N data from the sensors are transmitted hourly through a data-collection platform by satellite to the USGS National Water Information System database (USGS, 2020). Sensors were cleaned and calibrated monthly or as needed (Pellerin and others, 2013).

\section{Data Analysis}

All hydrological and water-quality data collected from 1990 to 2019 are stored in the USGS National Water Information System database (USGS, 2020) and have been summarized on a 5-year basis by Schulmeyer and others (1995), Schnoebelen and Schulmeyer (1996), Boyd and others (1999), Littin and Schnoebelen (2010), Littin (2012), and Meppelink and others (2019). Water-quality data used for analysis in this report are also available in Meppelink and Kalkhoff (2021).

A daily nitrate- $\mathrm{N}$ timeseries for the 1990-2019 study period was created to document the temporal trend in the Cedar River from discrete samples, high-frequency data, and estimated values. Nitrate- $\mathrm{N}$ concentrations in the Cedar River were from discrete samples collected by the Cedar Rapids Utilities Water Department (1990-2000), the Iowa Department of Natural Resources (2000-9), and the USGS (2009-19). The City of Cedar Rapids Utilities Water Department collected dip samples near the East well field (Jacob Donaghy, Cedar Rapids Utilities Water Quality Specialist, written commun., 2019). Monthly dip samples were collected for the Iowa Department of Natural Resources stream monitoring program at SW-1 (Iowa Department of Natural Resources, 2020b). The USGS data were from discrete samples and continuous nitrate- $\mathrm{N}$ data as previous described. Daily nitrate- $\mathrm{N}$ concentrations were estimated on days when discrete or high-frequency samples were missing using the fillMissing linear interpolation function in the smwrBase package (Lorenz, 2015), part of the USGS R library. The estimated values were limited to periods of missing data of 45 days or less. Estimated daily nitrate-N concentrations in the Cedar River are available in Meppelink and Kalkhoff (2021).

Water quality from samples collected from vertical production wells, Ranney production wells, and monitoring wells were compared using data from 2003 to 2019 to reduce differences caused by temporal variability and provide a more direct and accurate comparison of water-quality variability caused by well type. The Ranney production wells were first sampled in 2003, whereas the vertical production wells and monitoring wells were sampled beginning in 1993. The Ranney production wells sampled during the study were constructed during 2003-14. Comparison between water quality collected from the five Ranney production wells was made from 2014 to 2019 to eliminate variability caused by different sampling periods.

The statistical analysis for this report was run using SAS/ STAT software, version 9.4 of the SAS System for Windows. Data were statistically summarized to show central tendency (mean and median) and variability (minimum, 25th percentile, 75th percentile, and maximum values). In summarizing the data, the interquartile range (25th to 75 th percentiles) was used to define "normal" conditions.

Nonparametric tests were used to quantify water-quality differences between groups and to determine the significance of trends. Nonparametric tests were used because they do not depend on a normal distribution of the data, and they are resistant to the effect of outliers (Helsel and others, 2020). Water-quality differences between groups was determined using the nonparametric Kruskal-Wallis test. The Kruskal-Wallis test determines whether all groups have the same median, or whether at least one median was different. Follow-up tests of the Kruskal-Wallis test were made in SAS using pairwise two-sample comparisons to identify which groups have similar medians. Significant temporal trends in water quality constituents were identified using the nonparametric Kendall's tau test (Helsel and others, 2020). Results of all tests were considered significant at probabilities less than $0.05(p<0.05)$.

Analytical methods that changed during the study period generally resulted in lower laboratory reporting limits over time. Detection of a constituent for this report is a concentration greater than or equal to the analytical reporting limit irrespective of the analytical method reporting limit. Estimated concentrations are also considered to be a detection. The reporting limit concentration was used for censored less than results, and estimated values were used for statistical summary and analysis. 


\section{Hydrology of the Alluvial Aquifer}

Water in the alluvial aquifer originates from direct infiltration of precipitation into the aquifer; from groundwater flow from upland areas next to the alluvial aquifer; and, at times, from inflow from the Cedar River, floodplain oxbows and wetlands, and underlying bedrock aquifers. Water in the aquifer generally flows towards the Cedar River (Hansen, 1970; Turco and Buchmiller, 2004). Large-capacity supply wells screened in the alluvial aquifer may locally alter the normal groundwater flow paths inducing additional recharge from the Cedar River and underlying bedrock aquifers. A detailed description of hydrologic conditions in the study area are provided by Turco and Buchmiller (2004) and are summarized below.

\section{Movement Under Natural Conditions}

Recharge to the Cedar River alluvial aquifer occurs as infiltration of precipitation, infiltration of runoff from adjacent upland areas, leakage from underlying bedrock aquifers, and leakage from the Cedar River that includes bank storage. The alluvial aquifer receives an adequate amount of recharge from precipitation to maintain the water table above river stage (Hansen, 1970). Water from precipitation infiltrates the soil and moves vertically through the unsaturated zone to the water table (fig. 6A). Vertical movement is impeded at depth because of the presence of relatively impermeable confining unit consisting of till. Water then moves laterally downgradient towards the Cedar River (fig. 6A). Water in the shallow alluvial aquifer is young, having infiltrated within 5 to 30 years (Boyd, 1998). Water deeper in the aquifer is generally older (Boyd, 1998) because it moves along longer flow paths before discharging to the river. When the river stage is lower than the water table, the aquifer flows to the river. The Cedar River can receive as much as 80 percent of its annual streamflow from groundwater contributions (Squillace and others, 1996).

Under conditions of high streamflow in the Cedar River, the normal hydrologic gradient can be reversed at which time water from the river enters the adjacent alluvial aquifer via the streambank (fig. 6B). Through field observations and computer modeling, Squillace (1996) found that, during one period when the Cedar River rose more than $6 \mathrm{ft}$, river water infiltrated $100 \mathrm{ft}$ into the aquifer. Upon return to normal streamflow conditions, the hydrologic gradient is again reversed; water then flows back from the alluvial aquifer to the Cedar River (fig. 6A). Flow from the aquifer includes Cedar River water temporarily stored in the streambank during high flow (Squillace, 1996; Squillace and others, 1996). Modeling results indicated that bank storage water took as much as 5 weeks to drain back to the river after peak river stage (Squillace, 1996).

Water from the Cedar River periodically flows into backwater channels and overtops the streambanks spilling onto the floodplain in the study area. At these times, vertical
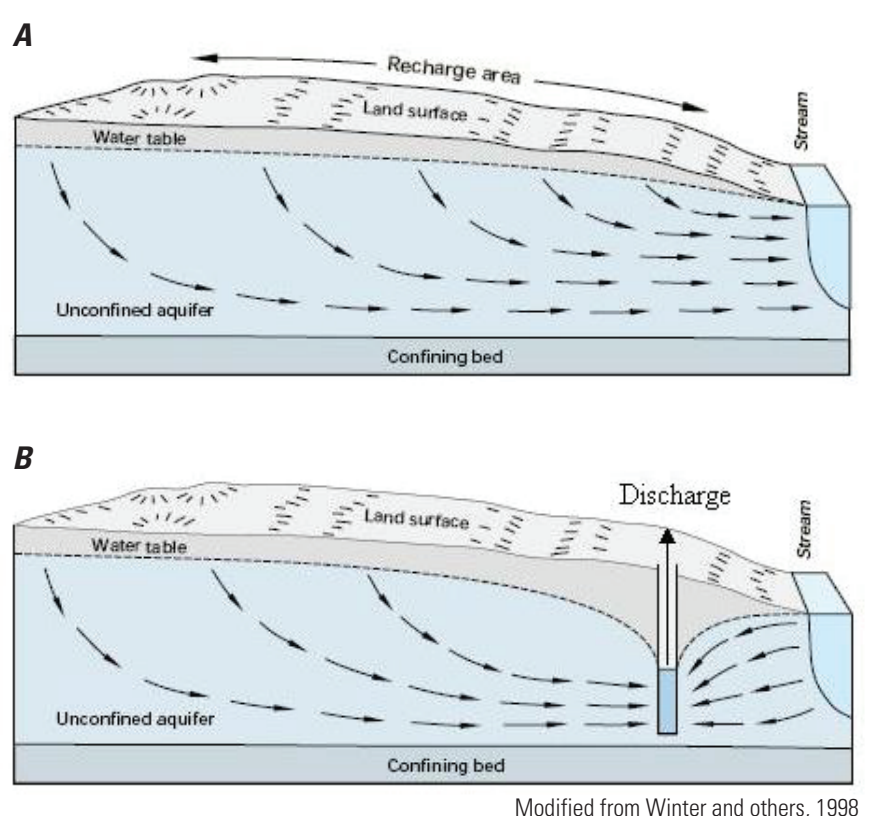

Figure 6. Conceptual flow through the Cedar River alluvial aquifer, Linn County, lowa. $A$, flow under normal conditions. $B$, flow under pumping conditions.

infiltration of floodwater into the alluvial aquifer can occur. Flow from the Cedar River to the floodplain is controlled by local topography, and all areas are not affected simultaneously. In the study area, water generally begins to flow into backwater oxbows and channels when streamflow in the Cedar River reaches about $17,000 \mathrm{ft}^{3} / \mathrm{s}$. When streamflow in the Cedar River reaches $30,000 \mathrm{ft}^{3} / \mathrm{s}$, the broader alluvial valley from Palo, Iowa, downstream to the Seminole well field is inundated (Iowa Flood Information System [IFIS], 2019). When flow in the Cedar River reaches $40,000 \mathrm{ft}^{3} / \mathrm{s}$, most of the alluvial floodplain is inundated in the study area (IFIS, 2019). During the 1990-2019 study period, streamflow in the Cedar River was great enough to flow into the backwater channels and wetlands during about 7.5 percent of the days and completely inundated the alluvial floodplain on about 1 percent of the days. Floodplain inundation occurred most often during June when flow in the Cedar River was normally the greatest.

Water and dissolved constituents in small tributaries that flow across alluvial deposits commonly lose water to the underlying aquifer (Buchmiller, 1995; Burkart and others, 1999).

Potential seepage may occur in three streams, Lone Tree, Silver, and Morgan Creek, that flow across the Cedar River alluvial deposits and enter from the west or south side of the river between Palo and Edgewood Road in Cedar Rapids. Similarly, two streams, Otter Creek, and McCloud Run, cross alluvial deposits and enter the Cedar River from the east or north side of the river in this same reach (fig. 1). 


\section{Movement Under Pumping Conditions}

Withdrawal of large quantities of water for residential and industrial purposes can alter the normal flow of water in the alluvial aquifer. Water moves towards production wells, drawing water from the alluvial aquifer, the river, and potentially from the underlying bedrock aquifer (fig. 4). Model estimates (Schulmeyer and Schnoebelen, 1998; Turco and Buchmiller, 2004) show that much of the water pumped by large-capacity production wells in Cedar Rapids originates from the Cedar River.

\section{Flow from the Cedar River}

During pumping, withdrawal results in water being pulled into the production well from surrounding areas of the alluvial aquifer and can induce flow from the Cedar River (figs. $6 B$ and 7) and underlying bedrock aquifer (Schnoebelen, 2008). Pumping can substantially draw down water levels near the well creating a localized cone of depression. When the cone of depression intercepts the Cedar River, induced infiltration of surface water to the alluvial aquifer will occur. A low-head dam (locally named the "Five-in-one" dam) downstream from the East well field is designed to maintain a minimum river altitude and to create a pool on the Cedar River (fig. 5). The pool acts as a source of recharge to the Cedar River alluvial aquifer in the East and West well fields (Schulmeyer and Schnoebelen, 1998).

During an intensive study of the interaction between the Cedar River and alluvial aquifer near Cedar Rapids vertical production well Sem10 (Schulmeyer, 1995), the water level in the alluvial aquifer closely followed the stage of the river (fig. 8). During pumping, the water level at vertical production well Sem10 ranged from 12 to $20 \mathrm{ft}$ below river stage, resulting in a steep gradient from the Cedar River towards well Seminole 10 (Schulmeyer, 1995). In contrast to the gradient reversal in unpumped areas (see description in "Movement Under Natural Conditions"), this gradient from the river to the aquifer was maintained irrespective of the river level. A gradient from the alluvial aquifer to the river occurred only when the production well was not pumping. Water levels in the alluvial aquifer rapidly rose when pumping ceased until they were similar to the level of the river (fig. 8)

\section{Flow from the Devonian Aquifer}

Before development of production wells in 1962, water from the alluvial aquifer near Cedar Rapids moved downward recharging the underlying bedrock aquifers in Devonian- and Silurian-aged formations (Hansen, 1970). After development, water began to move upward from the bedrock to the alluvial aquifer (Hansen, 1970). The upward hydrologic gradient continues to be observed because the alluvial aquifer was developed for water supply (Schulmeyer and Schnoebelen, 1998); however, the gradient is not consistent throughout the study area.

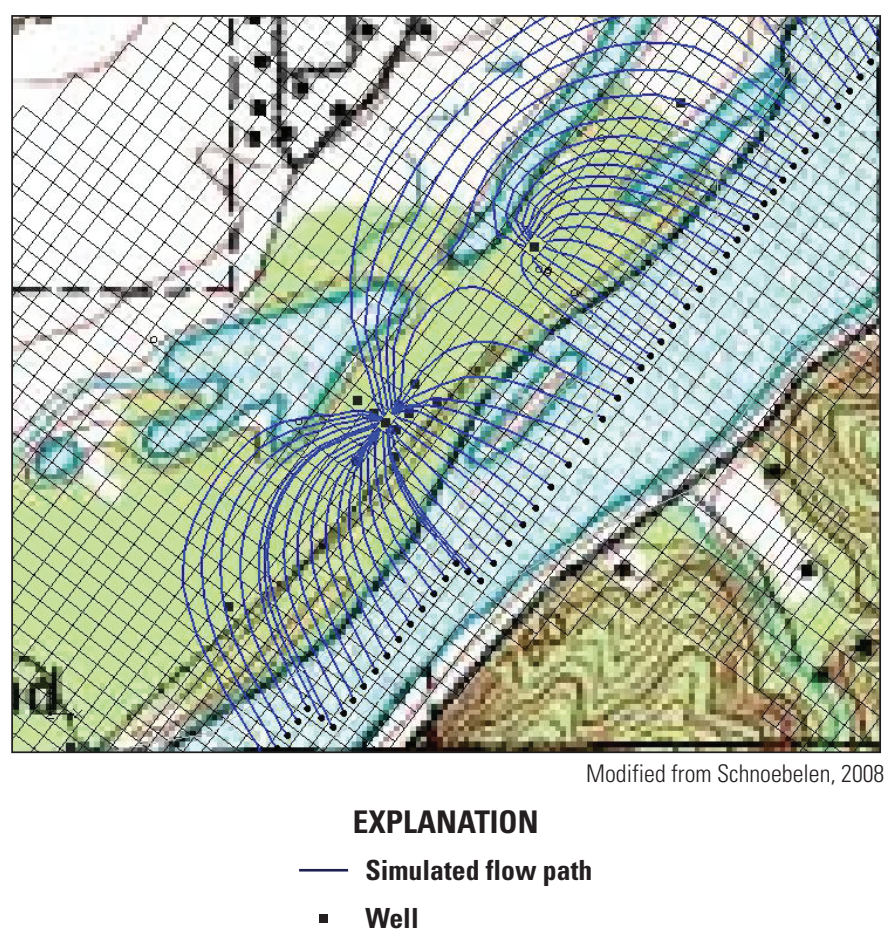

Figure 7. Simulated flow in the alluvial aquifer caused by pumping from a large-capacity production well.

Sequential measurement of co-located monitoring wells shows that the hydrologic gradient between the alluvial aquifer and underlying Devonian aquifer was not uniform in the well fields in Cedar Rapids. The gradient from the underlying bedrock was greater in the most upstream (Seminole) well field than in the East and West well fields (table 5). The substantial upward gradient from the Devonian aquifer to the alluvial aquifer (mean of $-8.10 \mathrm{ft}$ ) indicates that there was little flow between aquifers because of the shale confining unit (fig. 3) present in this part of the Seminole well field. There was little or no difference in water level (mean of $0.13 \mathrm{ft}$ ) in the West well field and only a small (mean of $-0.45 \mathrm{ft}$ ) water-level gradient from the Devonian aquifer to alluvial aquifer in the downstream East well field. Small or no hydraulic head differences indicate that the Devonian and alluvial aquifers are not separated by a confining unit (fig. 3) in these well fields. Throughout the study area, it was estimated, based on use of environmental tracers (Boyd, 1998) and from groundwater-flow models (Schulmeyer and Schnoebelen, 1998; Turco and Buchmiller, 2004), that 5 to 21 percent of water pumped from the production wells originated from the underlying bedrock aquifer, and higher proportions of water from the bedrock aquifer likely occur in wells in the West and East well fields. 


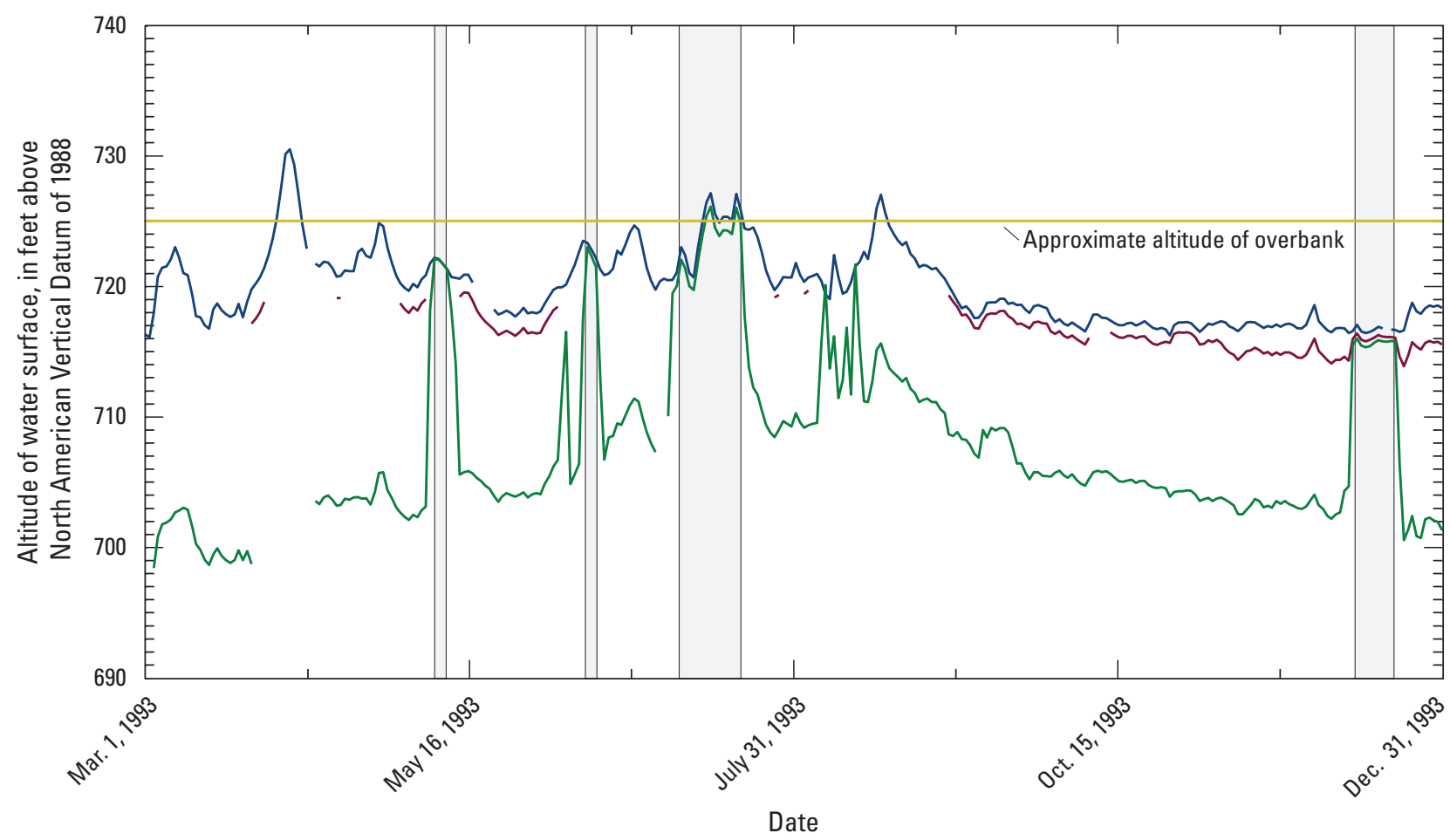

EXPLANATION

[Data from Schnoebelen and Schulmeyer, 1998]

Period when Sem10 was not pumping Monitoring well CRM4

Vertical production well Sem10

Cedar River

Figure 8. Water level in the alluvial aquifer (monitoring well CRM4 and vertical production well Sem10) in relation to the water level in the Cedar River (surface-water site SW-2), Linn County, lowa, March 1993 through December 1993.

Table 5. Hydrologic gradient between the Cedar River alluvial aquifer and underlying Devonian aquifer in the well fields in Cedar Rapids, Linn County, lowa, 1994-2001.

[Data are from the U.S. Geological Survey (USGS) National Water Information System database (USGS, 2020). Negative values are gradients from the Devonian aquifer to alluvial aquifer; positive values are gradients from the alluvial aquifer to Devonian aquifer; $n$, number of measurements; $\mathrm{ft}$, foot]

\begin{tabular}{|c|c|c|c|c|c|c|c|c|c|}
\hline Well field & $n$ & $\begin{array}{c}\text { Alluvial } \\
\text { aquifer } \\
\text { monitoring } \\
\text { well (fig. 5) }\end{array}$ & $\begin{array}{c}\text { Devonian } \\
\text { aquifer } \\
\text { monitoring } \\
\text { well (fig. 5) }\end{array}$ & \multicolumn{6}{|c|}{ Hydrologic gradient ( $\mathrm{ft}$ ) } \\
\hline Seminole & 62 & CRM4 & CRM6 & -13.68 & -9.42 & -8.10 & -7.62 & -6.75 & -3.99 \\
\hline West & 69 & CRM9 & CRM7 & -2.49 & -0.02 & 0.13 & 0.00 & 0.02 & 5.90 \\
\hline East & 68 & CRM1 & CRM12 & -0.72 & -0.53 & -0.45 & -0.46 & -0.41 & 1.14 \\
\hline
\end{tabular}




\section{Water Quality of the Alluvial Aquifer}

\section{Summary of Water Quality}

The water in the alluvial aquifer was generally a calcium bicarbonate type that was present in an environment of a narrow range of $\mathrm{pH}$ (7.1 to 7.4 standard units) with low dissolvedoxygen concentrations ( 75 percent of samples less than $[<]$ or equal to 2.1 milligrams per liter $[\mathrm{mg} / \mathrm{L}]$, table 6$)$. Calcium concentrations normally (25th to 75 th percentile) ranged from about 66.0 to $78.0 \mathrm{mg} / \mathrm{L}$ but, in some samples, were as small as $27.7 \mathrm{mg} / \mathrm{L}$ and as great as $203 \mathrm{mg} / \mathrm{L}$. Bicarbonate concentrations are normally within a small range from 229 to $272 \mathrm{mg} / \mathrm{L}$. Bicarbonate concentrations were as small as $149 \mathrm{mg} / \mathrm{L}$ and as great as $1,160 \mathrm{mg} / \mathrm{L}$ (table 6). Water in the alluvial aquifer also contained appreciable amounts of magnesium (mean of $20.8 \mathrm{mg} / \mathrm{L}$ ), sulfate (mean of $32.0 \mathrm{mg} / \mathrm{L}$ ), and chloride (mean of $23.6 \mathrm{mg} / \mathrm{L})$.

Nitrogen and phosphorus, which are naturally occurring elements and contaminants that originate from urban and agricultural runoff, were present in the alluvial aquifer in varying concentrations during the study period. Nitrate- $\mathrm{N}$ concentrations normally were present in concentrations ranging from 0.52 to $5.14 \mathrm{mg} / \mathrm{L}$, but 16 of the 783 samples from 11 wells exceeded the $10 \mathrm{mg} / \mathrm{L}$ MCL for drinking water (table 6; Meppelink and Kalkhoff, 2021). The median nitrate-N concentration in the Cedar River alluvial aquifer $(2.96 \mathrm{mg} / \mathrm{L})$ was similar to the median $(2.50 \mathrm{mg} / \mathrm{L})$ in samples obtained from production wells from 1986 to 1996 across Iowa (Schaap and Linhart, 1998). Ammonia-N, which was likely from degradation of organic matter, was generally present at concentrations $<0.14 \mathrm{mg} / \mathrm{L}$ (75th percentile) but did reach $22.6 \mathrm{mg} / \mathrm{L}$ in one sample. Twenty-nine percent of the samples had no detectable ammonia-N, but 6 percent of samples had ammonia-N concentrations of $1.0 \mathrm{mg} / \mathrm{L}$ or more. Ammonia- $\mathrm{N}$ in the Cedar River alluvial aquifer generally was present more frequently and at greater concentrations than those found in other production wells in alluvial aquifers across Iowa (Schaap and Linhart, 1998; Schilling, 2002).

Microbial oxidation of organic matter in parts of the alluvial aquifer produce anoxic conditions that favor dissolution of iron and manganese from aquifer materials (Boyd, 1999; Schnoebelen, 2008). Concentrations of iron and manganese in the alluvial aquifer frequently exceeded secondary drinkingwater standards. Iron concentrations normally ranged from 6.0 to 68.7 micrograms per liter ( $\mu \mathrm{g} / \mathrm{L}$; table 6 ), but the maximum concentration was substantially greater $(37,300 \mu \mathrm{g} / \mathrm{L})$. Iron concentrations exceeded the $300 \mu \mathrm{g} / \mathrm{L}$ secondary maximum contaminant level (SMCL; U.S. Environmental Protection Agency [EPA], 2018) in 11.0 percent of the samples. The exceedance of the SMCL of $50 \mu \mathrm{g} / \mathrm{L}$ (EPA, 2018) for manganese (66.8 percent) was much more frequent than iron (11.0 percent). Manganese concentrations commonly ranged from 25.3 to $428 \mu \mathrm{g} / \mathrm{L}$ (table 6). Previous monitoring (Schaap and Linhart, 1998) showed that exceedance of the iron and manganese SMCL was common in production wells withdrawing water from alluvial aquifers in Iowa.

Organic compounds were common in alluvial aquifer. The organic compounds include dissolved organic compounds from naturally occurring degradation of organic matter and synthetic man-made compounds (pesticides) used to control unwanted plants, insects, and fungi in urban and agricultural areas. Dissolved organic carbon (DOC), which is indicative of the naturally occurring compounds, was present in substantially greater abundance than the synthetic compounds. DOC concentrations in the alluvial aquifer normally ranged from 1.61 to $2.20 \mathrm{mg} / \mathrm{L}$ (table 6). The maximum DOC concentration was $6.61 \mathrm{mg} / \mathrm{L}$.

Several synthetic pesticides were detected in the alluvial aquifer at significantly smaller concentrations than the naturally occurring organic compounds (table 7). Triazine and acetoanolide preemergent herbicides were the most commonly detected compounds. Atrazine and one of its primary degradates, 2-Chloro-4-isopropylamino-6-amino-s-triazine (CIAT), was present in more than 90 percent of all samples from the alluvial aquifer. The maximum atrazine concentration $(2.71 \mu \mathrm{g} / \mathrm{L})$ in the alluvial aquifer was near the MCL of $3.0 \mu \mathrm{g} / \mathrm{L}$ for drinking water (EPA, 2018). Two acetoanalide herbicides, metolachlor and acetochlor, were detected in 87.7 and 27.5 percent of the samples, respectively. Only one other compound, the triazene herbicide prometon used for nonrow crop agricultural and urban weed control, was detected in more than 20 percent of the samples (table 7). A total of 32 pesticide compounds were detected in 1 or more samples during the study. Sixty-three additional compounds (app. 1) were never detected in samples during the study.

\section{Variability of Water Quality in the Alluvial Aquifer}

Concentrations of most water-quality constituents were highly variable during the study period. Variability can be caused by differences in where water is pumped from the aquifer because of how a well was constructed, can be caused by spatial differences because of differing geochemical conditions in the aquifer, and may vary temporally because of waterquality changes in source water from the Cedar River and the underlying Devonian aquifer.

\section{Water-Quality Differences Caused by Well Type}

The basic water chemistry of alluvial water pumped from vertical production wells, Ranney production wells, and monitoring wells was similar (table 8). Concentrations of all major ions and total dissolved solids were not significantly different ( $p$ greater than $[>]$ 0.05, Kruskal-Wallis Test) in samples from either production wells (vertical and Ranney) or monitoring wells. The total dissolved-solids concentration was greater than the SMCL in only 1.4 percent of all samples (fig. 9). 
Table 6. Summary of the physical properties, major ions, nutrients, and trace elements in the alluvial aquifer in the vicinity of Cedar Rapids, Linn County, lowa, 1990-2019.

[Data are summarized from the U.S. Geological Survey (USGS) National Water Information System database (USGS, 2020); search using the parameter code to see full parameter names, descriptions, and other data in the database. $n$, number of samples; $\mathrm{mg} / \mathrm{L}$, milligram per liter; $\mu \mathrm{S} / \mathrm{cm}$ at $25^{\circ} \mathrm{C}$, microsiemens per centimeter at 25 degrees Celsius; ${ }^{\circ} \mathrm{C}$, degrees $\mathrm{Celsius;} \mu \mathrm{g} / \mathrm{L}$, microgram per liter; $n$, number of samples; <, less than; E, estimated]

\begin{tabular}{|c|c|c|c|c|c|c|c|c|c|}
\hline \multirow[b]{2}{*}{ Constituent } & \multirow{2}{*}{$\begin{array}{l}\text { USGS parameter } \\
\text { code }\end{array}$} & \multirow[b]{2}{*}{ Unit of measurement } & \multirow[b]{2}{*}{$n$} & \multicolumn{6}{|c|}{ Concentration } \\
\hline & & & & Minimum & $\begin{array}{c}\text { 25th } \\
\text { percentile }\end{array}$ & Median & Mean & $\begin{array}{c}\text { 75th } \\
\text { percentile }\end{array}$ & Maximum \\
\hline \multicolumn{10}{|c|}{ Physical properties } \\
\hline Dissolved oxygen & 00300 & $\mathrm{mg} / \mathrm{L}$ & 738 & $<0.1$ & 0.2 & 0.5 & 1.66 & 2.1 & 14 \\
\hline $\mathrm{pH}$ & 00400 & Standard units & 795 & 5.5 & 7.1 & 7.2 & 7.2 & 7.4 & 8.2 \\
\hline Specific conductance & 00095 & $\mu \mathrm{S} / \mathrm{cm}$ at $25^{\circ} \mathrm{C}$ & 810 & 157 & 505 & 546 & 555 & 591 & 1,700 \\
\hline Temperature, water & 00010 & ${ }^{\circ} \mathrm{C}$ & 810 & 0.00 & 9.3 & 13.0 & 13.1 & 17.0 & 28.1 \\
\hline \multicolumn{10}{|c|}{ Major ions } \\
\hline Total dissolved solids & 70300 & $\mathrm{mg} / \mathrm{L}$ & 778 & 140 & 295 & 316.5 & 326 & 345 & 985 \\
\hline Calcium & 00915 & $\mathrm{mg} / \mathrm{L}$ & 779 & 27.7 & 65.7 & 72 & 73.6 & 78.3 & 203 \\
\hline Magnesium & 00925 & $\mathrm{mg} / \mathrm{L}$ & 779 & 4.5 & 18.1 & 20.7 & 20.8 & 22.7 & 61.8 \\
\hline Potassium & 00935 & $\mathrm{mg} / \mathrm{L}$ & 775 & $<0.10$ & 2.09 & 2.48 & 2.56 & 2.88 & 20.9 \\
\hline Sodium & 00930 & $\mathrm{mg} / \mathrm{L}$ & 780 & 2.60 & 8.84 & 10.7 & 11.4 & 13.0 & 128 \\
\hline Bicarbonate & 00453 & $\mathrm{mg} / \mathrm{L}$ & 604 & 149 & 229 & 252 & 259 & 272 & 1,160 \\
\hline Carbonate & 00452 & $\mathrm{mg} / \mathrm{L}$ & 568 & $<0.1$ & $<0.1$ & 0.20 & 0.21 & 0.30 & 2.2 \\
\hline Chloride & 00940 & $\mathrm{mg} / \mathrm{L}$ & 781 & 1.90 & 19.3 & 22.9 & 23.6 & 26.6 & 300 \\
\hline Sulfate & 00945 & $\mathrm{mg} / \mathrm{L}$ & 781 & $<0.1$ & 25.4 & 31.0 & 32.0 & 37.0 & 150 \\
\hline Fluoride & 00950 & $\mathrm{mg} / \mathrm{L}$ & 779 & E0.06 & 0.19 & 0.21 & 0.21 & 0.25 & 0.40 \\
\hline Silica & 00955 & $\mathrm{mg} / \mathrm{L}$ as $\mathrm{SiO}_{2}$ & 779 & $<0.04$ & 9.76 & 11.7 & 12.2 & 13.7 & 28.0 \\
\hline \multicolumn{10}{|c|}{ Nutrients } \\
\hline Ammonia $\left(\mathrm{NH}_{3}\right.$ plus $\left.\mathrm{NH}_{4}^{+}\right)$ & 00608 & $\mathrm{mg} / \mathrm{L}$ as $\mathrm{N}$ & 783 & E0.006 & 0.02 & 0.04 & 0.38 & 0.14 & 22.6 \\
\hline Nitrate plus nitrite & 00631 & $\mathrm{mg} / \mathrm{L}$ as $\mathrm{N}$ & 783 & $<0.02$ & 0.52 & 2.96 & 3.20 & 5.14 & 15.0 \\
\hline Nitrate & 00618 & $\mathrm{mg} / \mathrm{L}$ as $\mathrm{N}$ & 764 & $<0.02$ & 0.45 & 3.00 & 3.21 & 5.16 & 15.0 \\
\hline Nitrite & 00613 & $\mathrm{mg} / \mathrm{L}$ as $\mathrm{N}$ & 765 & $<0.001$ & 0.005 & 0.009 & 0.016 & 0.012 & 0.443 \\
\hline Orthophosphate & 00671 & $\mathrm{mg} / \mathrm{L}$ as $\mathrm{P}$ & 782 & $<0.004$ & 0.03 & 0.053 & 0.067 & 0.09 & 0.73 \\
\hline Dissolved organic carbon & 00681 & $\mathrm{mg} / \mathrm{L}$ & 721 & 0.20 & 1.61 & 1.85 & 1.97 & 2.20 & 6.61 \\
\hline \multicolumn{10}{|c|}{ Trace elements } \\
\hline Bromide & 71870 & $\mathrm{mg} / \mathrm{L}$ & 755 & $<0.01$ & 0.028 & 0.032 & 0.85 & 0.04 & 610 \\
\hline Iron & 01046 & $\mu \mathrm{g} / \mathrm{L}$ & 778 & $<2.00$ & 6.00 & 10.0 & 535 & 68.7 & 37,300 \\
\hline Manganese & 01056 & $\mu \mathrm{g} / \mathrm{L}$ & 777 & $<0.16$ & 25.3 & 150 & 408 & 428 & 7,500 \\
\hline Boron & 01020 & $\mu \mathrm{g} / \mathrm{L}$ & 741 & 9.20 & 23.0 & 27.0 & 30.2 & 31.0 & 213 \\
\hline
\end{tabular}


Table 7. Occurrence of pesticide compounds in the alluvial aquifer, Devonian aquifer, and Cedar River near Cedar Rapids, Linn County, lowa, $1990-2019$.

[Data are summarized from the U.S. Geological Survey (USGS) National Water Information System database (USGS, 2020); search using the parameter code to see full parameter names, descriptions, and other data in the database. $n$, number of samples; $\mu \mathrm{g} / \mathrm{L}$, microgram per liter; DCPA, dimethyl tetrachloroterephthalate; EPTC, S-ethyl dipropylthiocarbamate]

\begin{tabular}{|c|c|c|c|c|c|c|c|c|c|c|}
\hline \multirow[b]{2}{*}{ Pesticide compound } & \multirow[b]{2}{*}{$\begin{array}{c}\text { USGS } \\
\text { parameter } \\
\text { code }\end{array}$} & \multicolumn{3}{|c|}{ Alluvial aquifer } & \multicolumn{3}{|c|}{ Devonian aquifer } & \multicolumn{3}{|c|}{ Cedar River } \\
\hline & & $n$ & $\begin{array}{l}\text { Detection } \\
\text { rate } \\
\text { (percent) }\end{array}$ & $\begin{array}{c}\text { Maximum } \\
\text { concentration } \\
(\mu \mathrm{g} / \mathrm{L})\end{array}$ & $n$ & $\begin{array}{l}\text { Detection } \\
\text { rate } \\
\text { (percent) }\end{array}$ & $\begin{array}{c}\text { Maximum } \\
\text { concentration } \\
(\mu \mathrm{g} / \mathrm{L})\end{array}$ & $n$ & $\begin{array}{l}\text { Detection } \\
\text { rate } \\
\text { (percent) }\end{array}$ & $\begin{array}{c}\text { Maximum } \\
\text { concentration } \\
(\mu \mathrm{g} / \mathrm{L})\end{array}$ \\
\hline Atrazine & 39632 & 761 & 96.2 & 2.71 & 30 & 20.0 & 0.09 & 122 & 99.2 & 8.16 \\
\hline $\begin{array}{l}\text { 2-Chloro-4-isopropylamino-6-amino-s- } \\
\text { triazine (CIAT) }\end{array}$ & 04040 & 761 & 94.1 & 0.50 & 30 & 10.0 & 0.05 & 122 & 96.7 & 0.66 \\
\hline Metolachlor & 39415 & 757 & 87.7 & 1.2 & 30 & 16.7 & 0.08 & 122 & 94.3 & 9.67 \\
\hline Acetochlor & 49260 & 683 & 27.5 & 0.30 & 11 & 0.00 & $<0.05$ & 104 & 53.8 & 3.83 \\
\hline $\begin{array}{l}\text { 2-Chloro-6-ethylamino-4-amino-s-triazine } \\
\text { (CEAT) }\end{array}$ & 04038 & 675 & 28.9 & 0.26 & 27 & 0.00 & $<0.05$ & 99 & 39.4 & 0.45 \\
\hline trans-Propiconazole & 79847 & 83 & 8.43 & 0.04 & 3 & 0.00 & $<0.018$ & 16 & 37.5 & 0.04 \\
\hline Prometon & 04037 & 759 & 21.5 & 0.10 & 30 & 3.33 & 0.05 & 120 & 18.3 & 0.12 \\
\hline DCPA & 82682 & 86 & 5.81 & 0.008 & 3 & 0.00 & $<0.0076$ & 26 & 26.9 & 0.008 \\
\hline cis-Propiconazole & 79846 & 83 & 2.41 & 0.04 & 3 & 0.00 & $<0.008$ & 16 & 25.0 & 0.04 \\
\hline Metribuzin & 82630 & 760 & 6.71 & 0.07 & 30 & 3.33 & 0.07 & 120 & 14.2 & 0.954 \\
\hline Propazine & 38535 & 670 & 7.01 & 0.05 & 27 & 0.00 & $<0.05$ & 98 & 13.3 & 0.08 \\
\hline Simazine & 04035 & 761 & 6.70 & 0.05 & 30 & 0.00 & $<0.05$ & 122 & 10.7 & 0.10 \\
\hline Desulfinylfipronil & 62170 & 83 & 3.61 & 0.012 & 3 & 0.00 & $<0.012$ & 22 & 13.6 & 0.012 \\
\hline Cyanazine & 04041 & 761 & 4.60 & 0.67 & 30 & 0.00 & $<0.2$ & 122 & 11.5 & 2.07 \\
\hline Alachlor & 46342 & 761 & 0.92 & 0.18 & 30 & 0.00 & $<0.05$ & 122 & 13.9 & 1.42 \\
\hline Metalaxyl & 61596 & 82 & 0.00 & $<0.087$ & 3 & 0.00 & $<0.014$ & 16 & 12.5 & 0.024 \\
\hline 2-Ethyl-6-methylaniline & 61620 & 83 & 2.41 & 0.01 & 3 & 0.00 & $<0.01$ & 16 & 6.25 & 0.01 \\
\hline ЕРTC & 82668 & 86 & 0.00 & $<0.008$ & 3 & 0.00 & $<0.008$ & 26 & 7.69 & 0.008 \\
\hline Terbuthylazine & 04022 & 84 & 1.19 & 0.02 & 3 & 0.00 & $<0.008$ & 17 & 5.88 & 0.02 \\
\hline Pendimethalin & 82683 & 87 & 1.15 & 0.05 & 3 & 0.00 & $<0.012$ & 26 & 3.85 & 0.028 \\
\hline Fipronil & 62166 & 81 & 0.00 & $<0.018$ & 3 & 0.00 & $<0.018$ & 22 & 4.55 & 0.018 \\
\hline Diazinon & 39572 & 86 & 0.00 & $<0.018$ & 3 & 0.00 & $<0.008$ & 26 & 3.85 & 0.008 \\
\hline Tefluthrin & 61606 & 83 & 3.61 & 0.014 & 3 & 0.00 & $<0.014$ & 16 & 0.00 & $<0.014$ \\
\hline Fipronil sulfide & 62167 & 83 & 2.41 & 0.016 & 3 & 0.00 & $<0.016$ & 22 & 0.00 & $<0.016$ \\
\hline Ametryn & 38401 & 665 & 1.50 & 0.05 & 26 & 0.00 & $<0.05$ & 93 & 0.00 & $<0.05$ \\
\hline Cycloate & 04031 & 570 & 0.00 & $<0.05$ & 8 & 0.00 & $<0.05$ & 72 & 1.39 & 0.05 \\
\hline Bromacil & 04029 & 573 & 0.00 & $<1.0$ & 8 & 0.00 & $<0.05$ & 75 & 1.33 & 1.00 \\
\hline
\end{tabular}


Table 7. Occurrence of pesticide compounds in the alluvial aquifer, Devonian aquifer, and Cedar River near Cedar Rapids, Linn County, lowa, 1990-2019.-Continued

[Data are summarized from the U.S. Geological Survey (USGS) National Water Information System database (USGS, 2020); search using the parameter code to see full parameter names, descriptions, and other data in the database. $n$, number of samples; $\mu \mathrm{g} / \mathrm{L}$, microgram per liter; DCPA, dimethyl tetrachloroterephthalate; EPTC, S-ethyl dipropylthiocarbamate]

\begin{tabular}{|c|c|c|c|c|c|c|c|c|c|c|}
\hline \multirow[b]{2}{*}{ Pesticide compound } & \multirow[b]{2}{*}{$\begin{array}{c}\text { USGS } \\
\text { parameter } \\
\text { code }\end{array}$} & \multicolumn{3}{|c|}{ Alluvial aquifer } & \multicolumn{3}{|c|}{ Devonian aquifer } & \multicolumn{3}{|c|}{ Cedar River } \\
\hline & & $n$ & $\begin{array}{l}\text { Detection } \\
\text { rate } \\
\text { (percent) }\end{array}$ & $\begin{array}{c}\text { Maximum } \\
\text { concentration } \\
(\mu \mathrm{g} / \mathrm{L})\end{array}$ & $n$ & $\begin{array}{l}\text { Detection } \\
\text { rate } \\
\text { (percent) }\end{array}$ & $\begin{array}{c}\text { Maximum } \\
\text { concentration } \\
(\mu \mathrm{g} / \mathrm{L})\end{array}$ & $n$ & $\begin{array}{l}\text { Detection } \\
\text { rate } \\
\text { (percent) }\end{array}$ & $\begin{array}{c}\text { Maximum } \\
\text { concentration } \\
(\mu \mathrm{g} / \mathrm{L})\end{array}$ \\
\hline Trifluralin & 82661 & 86 & 1.16 & 0.018 & 3 & 0.00 & $<0.018$ & 26 & 0.00 & $<0.018$ \\
\hline Benfluralin & 82673 & 86 & 1.16 & 0.014 & 3 & 0.00 & $<0.014$ & 26 & 0.00 & $<0.014$ \\
\hline cis-Permethrin & 82687 & 86 & 1.16 & 0.012 & 3 & 0.00 & $<0.012$ & 26 & 0.00 & $<0.014$ \\
\hline Trifluralin & 04023 & 565 & 0.53 & 0.05 & 8 & 0.00 & $<0.05$ & 71 & 0.00 & $<0.05$ \\
\hline Terbacil & 04032 & 570 & 0.53 & 0.05 & 8 & 0.00 & $<0.05$ & 72 & 0.00 & $<0.09$ \\
\hline Carboxin & 04027 & 548 & 0.18 & 0.50 & 7 & 0.00 & $<0.05$ & 67 & 0.00 & $<0.10$ \\
\hline Propachlor & 04024 & 600 & 0.17 & 0.05 & 8 & 0.00 & $<0.05$ & 88 & 0.00 & $<0.05$ \\
\hline Hexazinone & 04025 & 653 & 0.15 & 0.50 & 11 & 0.00 & $<0.05$ & 88 & 0.00 & $<0.50$ \\
\hline Prometryn & 04036 & 752 & 0.13 & 0.05 & 29 & 0.00 & $<0.05$ & 109 & 0.00 & $<0.05$ \\
\hline
\end{tabular}


Table 8. Summary of the physical properties, major ions, nutrients, and trace elements in relation to well type in the alluvial aquifer near Cedar Rapids, Linn County, lowa, 1990-2019.

[Data are summarized from the U.S. Geological Survey (USGS) National Water Information System database (USGS, 2020); search using the parameter code to see full parameter names, descriptions, and other data in the database. See well locations on figure 5. $n$, number of samples; Min, minimum; Med, median; Max, maximum; mg/L, milligram per liter; $\mu \mathrm{S} / \mathrm{cm}$ at $25^{\circ} \mathrm{C}$, microsiemens per centimeter at 25 degrees Celsius; ${ }^{\circ} \mathrm{C}$, degree Celsius; $\mathrm{SiO}_{2}$, silicon dioxide; $\mathrm{NH}_{4}{ }^{+}$, ammonium; $\mathrm{N}$, nitrogen; $\mathrm{P}$, phosphorus; $\mu \mathrm{g} / \mathrm{L}$, microgram per liter]

\begin{tabular}{|c|c|c|c|c|c|c|c|c|c|c|c|c|c|c|}
\hline \multirow{2}{*}{ Constituent } & \multirow{2}{*}{$\begin{array}{c}\text { USGS } \\
\text { parameter } \\
\text { code }\end{array}$} & \multirow{2}{*}{$\begin{array}{c}\text { Unit of } \\
\text { measurement }\end{array}$} & \multicolumn{4}{|c|}{ Vertical production wells } & \multicolumn{4}{|c|}{ Ranney production wells } & \multicolumn{4}{|c|}{ Monitoring wells } \\
\hline & & & $n$ & Min & Med & $\operatorname{Max}$ & $n$ & Min & Med & Max & $n$ & Min & Med & Max \\
\hline \multicolumn{15}{|c|}{ Physical properties } \\
\hline $\begin{array}{r}\text { Dissolved } \\
\text { oxygen }\end{array}$ & 00300 & $\mathrm{mg} / \mathrm{L}$ & 149 & 0.1 & 0.8 & 10.8 & 249 & 0.1 & 1.4 & 9.7 & 340 & $<0.1$ & 0.2 & 14 \\
\hline $\mathrm{pH}$ & 00400 & Standard units & 156 & 6.5 & 7.2 & 7.8 & 257 & 6.5 & 7.3 & 7.7 & 382 & 5.5 & 7.2 & 8.2 \\
\hline $\begin{array}{l}\text { Specific con- } \\
\text { ductance }\end{array}$ & 00095 & $\mu \mathrm{S} / \mathrm{cm}$ at $25^{\circ} \mathrm{C}$ & 162 & 6.0 & 543 & 809 & 261 & 413 & 548 & 709 & 387 & 6.0 & 552 & 1,700 \\
\hline $\begin{array}{l}\text { Temperature, } \\
\text { water }\end{array}$ & 00010 & ${ }^{\circ} \mathrm{C}$ & 161 & 0.4 & 12.9 & 24.0 & 261 & 2.0 & 14.5 & 28.1 & 388 & 0.0 & 12.2 & 26.1 \\
\hline \multicolumn{15}{|c|}{ Major ions } \\
\hline $\begin{array}{l}\text { Total dissolved } \\
\text { solids }\end{array}$ & 70300 & $\mathrm{mg} / \mathrm{L}$ & 156 & 217 & 312 & 450 & 254 & 242 & 316 & 467 & 368 & 140 & 321 & 985 \\
\hline Calcium & 00915 & $\mathrm{mg} / \mathrm{L}$ & 151 & 27.7 & 73.0 & 110 & 254 & 47.1 & 72.4 & 90.3 & 374 & 29.0 & 70.2 & 203 \\
\hline Magnesium & 00925 & $\mathrm{mg} / \mathrm{L}$ & 151 & 15.0 & 20.9 & 36.0 & 254 & 14.6 & 21.2 & 28.2 & 374 & 4.5 & 20.0 & 61.8 \\
\hline Potassium & 00935 & $\mathrm{mg} / \mathrm{L}$ & 151 & 1.40 & 2.54 & 5.10 & 254 & 1.51 & 2.49 & 4.04 & 370 & 0.10 & 2.38 & 20.9 \\
\hline Sodium & 00930 & $\mathrm{mg} / \mathrm{L}$ & 152 & 6.5 & 10.7 & 21.2 & 254 & 7.0 & 11.1 & 26.4 & 374 & 2.6 & 10.0 & 128 \\
\hline Bicarbonate & 00453 & $\mathrm{mg} / \mathrm{L}$ & 108 & 201 & 252 & 522 & 241 & 150 & 251 & 312 & 255 & 149 & 252 & 1,160 \\
\hline Carbonate & 00452 & $\mathrm{mg} / \mathrm{L}$ & 106 & $<0.1$ & 0.2 & 2.0 & 231 & $<0.1$ & 0.3 & 1.7 & 231 & $<0.1$ & $<0.1$ & 2.2 \\
\hline Chloride & 00940 & $\mathrm{mg} / \mathrm{L}$ & 153 & 13.0 & 23.0 & 39.6 & 254 & 13.1 & 24.6 & 47.3 & 374 & 1.9 & 21.7 & 300 \\
\hline Sulfate & 00945 & $\mathrm{mg} / \mathrm{L}$ & 153 & 19.0 & 31.9 & 51.9 & 254 & 16.1 & 29.9 & 56.0 & 374 & 0.10 & 32.0 & 150 \\
\hline Fluoride & 00950 & $\mathrm{mg} / \mathrm{L}$ & 151 & 0.10 & 0.21 & 0.33 & 254 & 0.14 & 0.21 & 0.30 & 374 & 0.06 & 0.20 & 0.40 \\
\hline Silica & 00955 & $\mathrm{mg} / \mathrm{L}$ as $\mathrm{SiO}_{2}$ & 151 & 0.04 & 11.1 & 16.0 & 254 & 7.00 & 11.8 & 17.5 & 374 & 5.05 & 12.0 & 28.0 \\
\hline \multicolumn{15}{|c|}{ Nutrients } \\
\hline $\begin{array}{c}\text { Ammonia }\left(\mathrm{NH}_{3}\right. \\
\left.\text { plus } \mathrm{NH}_{4}^{+}\right)\end{array}$ & 00608 & $\mathrm{mg} / \mathrm{L}$ as $\mathrm{N}$ & 157 & 0.01 & 0.08 & 2.0 & 253 & 0.006 & 0.05 & 1.74 & 373 & 0.01 & 0.04 & 22.6 \\
\hline $\begin{array}{l}\text { Nitrate plus } \\
\text { nitrite }\end{array}$ & 00631 & $\mathrm{mg} / \mathrm{L}$ as $\mathrm{N}$ & 157 & 0.05 & 3.00 & 8.30 & 253 & 0.06 & 4.21 & 11.4 & 373 & 0.02 & 0.79 & 15.0 \\
\hline Nitrate & 00618 & $\mathrm{mg} / \mathrm{L}$ as $\mathrm{N}$ & 139 & 0.05 & 3.08 & 8.29 & 252 & 0.058 & 4.21 & 11.4 & 373 & 0.022 & 0.715 & 15.0 \\
\hline Nitrite & 00613 & $\mathrm{mg} / \mathrm{L}$ as $\mathrm{N}$ & 139 & 0.001 & 0.008 & 0.055 & 253 & 0.001 & 0.005 & 0.074 & 373 & 0.001 & 0.010 & 0.443 \\
\hline Orthophosphate & 00671 & $\mathrm{mg} / \mathrm{L}$ as $\mathrm{P}$ & 157 & 0.005 & 0.032 & 0.150 & 253 & 0.01 & 0.061 & 0.234 & 372 & 0.010 & 0.070 & 0.730 \\
\hline
\end{tabular}


Table 8. Summary of the physical properties, major ions, nutrients, and trace elements in relation to well type in the alluvial aquifer near Cedar Rapids, Linn County, lowa, 1990-2019.-Continued

Data are summarized from the U.S. Geological Survey (USGS) National Water Information System database (USGS, 2020); search using the parameter code to see full parameter names, descriptions, and other data in the database. See well locations on figure 5. $n$, number of samples; Min, minimum; Med, median; Max, maximum; mg/L, milligram per liter; $\mu \mathrm{S} / \mathrm{cm}$ at $25^{\circ} \mathrm{C}$, microsiemens per centimeter at 25 degrees Celsius; ${ }^{\circ} \mathrm{C}$, degree Celsius; $\mathrm{SiO}_{2}$, silicon dioxide; $\mathrm{NH}_{4}^{+}$, ammonium; $\mathrm{N}$, nitrogen; $\mathrm{P}$, phosphorus; $\mu \mathrm{g} / \mathrm{L}$, microgram per liter]

\begin{tabular}{|c|c|c|c|c|c|c|c|c|c|c|c|c|c|c|}
\hline \multirow{2}{*}{ Constituent } & \multirow{2}{*}{$\begin{array}{c}\text { USGS } \\
\text { parameter } \\
\text { code }\end{array}$} & \multirow{2}{*}{$\begin{array}{c}\text { Unit of } \\
\text { measurement }\end{array}$} & \multicolumn{4}{|c|}{ Vertical production wells } & \multicolumn{4}{|c|}{ Ranney production wells } & \multicolumn{4}{|c|}{ Monitoring wells } \\
\hline & & & $n$ & Min & Med & Max & $n$ & Min & Med & Max & $n$ & Min & Med & Max \\
\hline \multicolumn{15}{|c|}{ Nutrients-Continued } \\
\hline $\begin{array}{c}\text { Dissolved } \\
\text { organic } \\
\text { carbon }\end{array}$ & 00681 & $\mathrm{mg} / \mathrm{L}$ & 133 & 1.41 & 1.82 & 3.37 & 252 & 1.16 & 1.79 & 3.88 & 336 & 0.40 & 2.00 & 6.61 \\
\hline \multicolumn{15}{|c|}{ Trace elements } \\
\hline Bromide & 71870 & $\mathrm{mg} / \mathrm{L}$ & 137 & 0.018 & 0.036 & 0.20 & 248 & 0.010 & 0.033 & 0.20 & 370 & 0.010 & 0.030 & 610 \\
\hline Iron & 01046 & $\mu \mathrm{g} / \mathrm{L}$ & 150 & 3.00 & 69.6 & 3,400 & 254 & 3.00 & 6.00 & 685 & 374 & 2.00 & 10.0 & 37,300 \\
\hline Manganese & 01056 & $\mu \mathrm{g} / \mathrm{L}$ & 150 & 3.00 & 208 & 1,300 & 253 & 0.20 & 59.2 & 1,370 & 374 & 0.16 & 228 & 7,500 \\
\hline Boron & 01020 & $\mu \mathrm{g} / \mathrm{L}$ & 134 & 10 & 26 & 50 & 254 & 14 & 26 & 47 & 353 & 9.2 & 30 & 213 \\
\hline
\end{tabular}



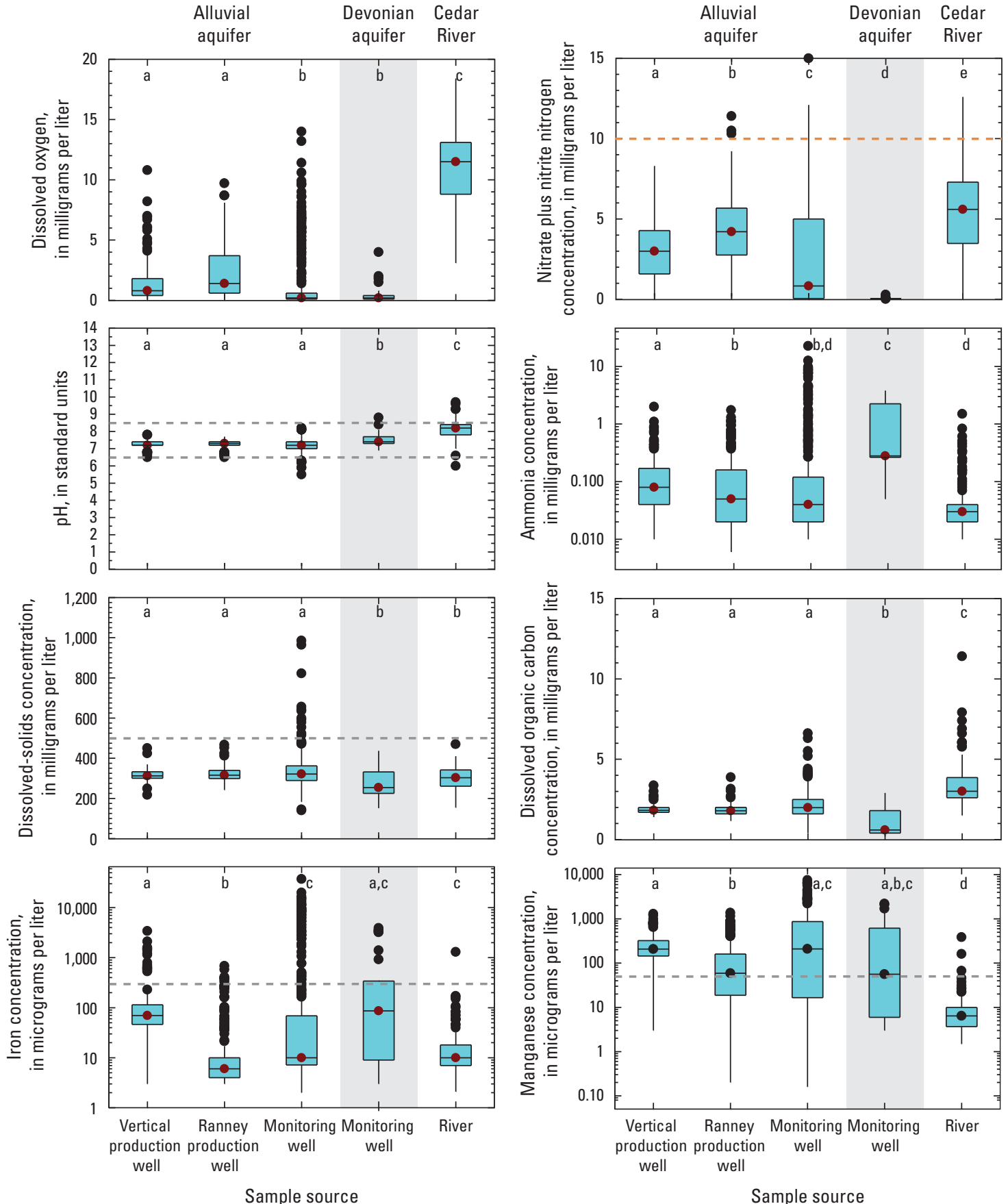

EXPLANATION

[Data from the U.S. Geological Survey (USGS) National Water Information System database (USGS, 2020); a, b, c, d, and e indicate statistically similar groups. Different letters at the top of each plot indicate statistically significant differences in characteristics between sample sources, whereas same letters indicate sample sources that are not significantly different]

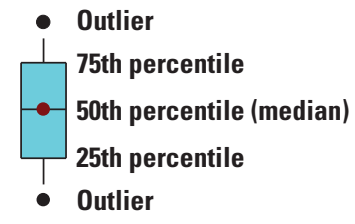

- - - - Drinking water maximum contaminant level

Secondary maximum contaminant level

(U.S. Environmental Protection Agency, 2018)

Figure 9. Comparison of water chemistry in relation to well type and potential source water to the alluvial aquifer, Linn County, lowa, 1990-2019. 
Water from the Ranney production wells was significantly ( $p<0.05$, Kruskal-Wallis Test) more oxygenated than water from the vertical production wells screened through the entire alluvial aquifer and from monitoring wells generally screened in a small, 5.0- to $10.0-\mathrm{ft}$ sections of the alluvial aquifer. A similar pattern was found for nitrate-N concentrations (fig. 9, table 8). In contrast, the iron concentrations in samples from the Ranney wells and the monitoring wells were significantly less than in the vertical wells (table $8 ; p<0.05$, Kruskal-Wallis Test). Manganese concentrations in the Ranney production wells were significantly ( $p<0.05$, Kruskal-Wallis Test) less than in vertical production and monitoring wells. DOC concentrations were lowest ( $p<0.05$, Kruskal-Wallis Test) in the highly oxygenated Ranney production well samples (fig. 9, table 8).
For pesticide compounds, the rate of detection varied depending on the compound but varied by $<10$ percent between well types for the same pesticide (fig. 10). Although small differences were found between well type, pesticides tended to be present slightly more often in Ranney production wells than other well types. Several compounds, including two fipronil degradates (desulfinyfipronil and fipronil sulfide), were only detected in Ranney production well samples (fig. 10). Atrazine and one of its degradate compounds (CIAT) were detected in all samples collected from the production wells during 2003-19. Two acetanilide pesticides, metolachlor and acetochlor, were present more frequently in the vertical production and Ranney production wells than in monitoring wells. Metolachlor was present in more than 98 percent of samples from both types of production wells and in 83 percent
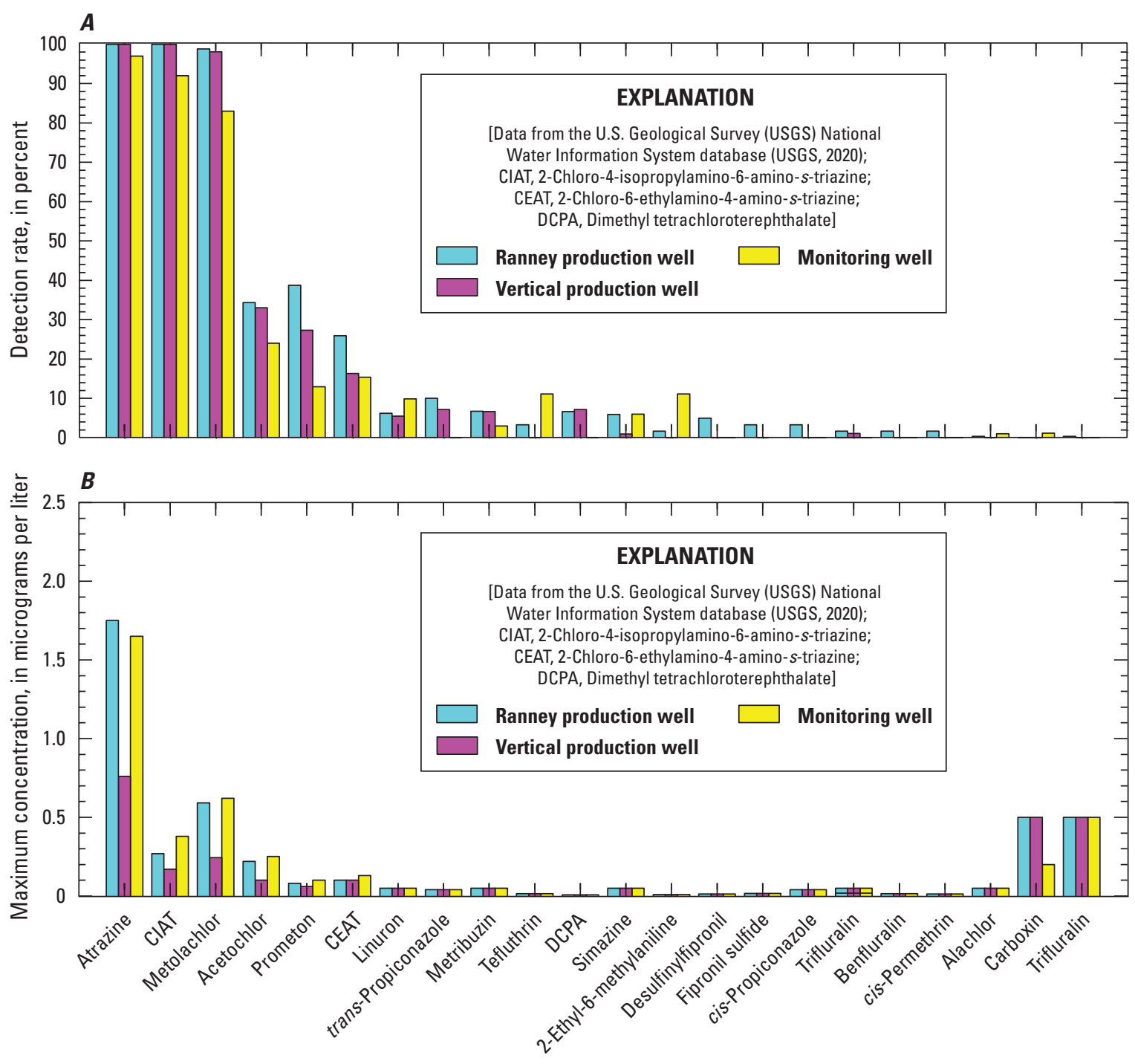

Pesticide compound

Figure 10. Detected pesticide compounds from Ranney and vertical production wells and monitoring wells screened in the Cedar River alluvial aquifer near Cedar Rapids, Linn County, lowa, 2003-19. A, detection rates. $B$, maximum concentration. 
of monitoring well samples. Acetochlor was present in more than 30 percent of samples from the vertical production and Ranney production wells but was present in only 24 percent of the monitoring well samples (fig. 10). The triazine degradate, 2-Chloro-6-ethylamino-4-amino-s-triazine (CEAT), was the only other compound present in 15 or more percent of samples.

Although detected on a similar frequency, maximum concentrations of many pesticides were greater in samples from the Ranney production wells and the monitoring wells compared to the vertical production wells, particularly for the most frequently detected pesticides (fig. 10). One reason for this pattern may be that the construction and large pumping rates of Ranney production wells may draw more water from the Cedar River than the vertical production wells whose water originates from more diverse sources. However, this does not explain why monitoring wells have similarly high maximum concentrations. Thus, the location of the Ranney production wells and monitoring wells near the Cedar River may be highly affected by induced infiltration from the river.

\section{Spatial Variability in Alluvial Water Quality}

Water-quality variability in the alluvial aquifer may also be caused by variations in redox conditions of the aquifer. A gradient in the concentrations of several redox-affected constituents occurs in groundwater from the Seminole well field to the West well field, following the upstream to downstream gradient of the overlying Cedar River. An example of this change is illustrated with data from the large-capacity Ranney production wells along a 3-river-mile reach of the Cedar River, from Ran6 in the northern end of the Seminole well field to Ran3 in the western part of the West well field (fig. 11). With the exception of the most upstream well (Ran6), concentrations of dissolved oxygen, nutrients (nitrate- $\mathrm{N}$, and ammonia-N), iron, and manganese gradually increased or decreased from the Seminole well field to the West well field, located upstream to downstream along the Cedar River, respectively (fig. 11). A few water-quality characteristics, including dissolved-solids concentrations and DOC, do not show a significant gradient $(p<0.05$, Kruskal-Wallis Test) across the five Ranney wells (fig. 11).

In the Seminole well field, median dissolved oxygen and nitrate $-\mathrm{N}$ concentrations generally decreased when moving from more upstream to more downstream wells. Organic matter was abundant throughout this reach of the alluvial aquifer, and median concentrations were not significantly different $(p>0.05$, Kruskal-Wallis Test) in the Ranney production wells. Although median dissolved-oxygen concentrations were not significantly $(p>0.05$, Kruskal-Wallis Test) different, there was a general downward trend from the upstream Ran6 through the Seminole well field downstream to Ran3 (fig. 11). With the exception in Ran6, median ammonia- $\mathrm{N}$, nitrite- $\mathrm{N}$, and manganese concentrations increased significantly ( $\mathrm{p}<0.05$, Kruskal-Wallis Test) from Ranney production wells Ran1 and Ran2 (fig. 5) in the Seminole well field south of the Cedar River downstream to Ran3 in the West well field. Concentrations of nitrite-N and iron are greatest in the most upstream Ran6. Manganese concentrations in samples from Ran6 are similar to those in the most downstream wells, Ran3 and Ran4.

The upstream to downstream water-quality gradients were also seen in the alluvial aquifer in a dataset collected by the Cedar Rapids Utilities Water Department on May 14-17, 2012, from 38 supply wells in the 5.2-river-mile reach of the Cedar Rapids wells fields (fig. 12). The dataset included vertical production and Ranney production wells in all three well fields. Nitrate-N, normally found in oxygenated environments, had the greatest concentrations in the most upstream wells in the Seminole well field and the lowest concentrations in the most downstream wells in the East well field (fig. 12). Nitrate-N concentrations were $>5.0 \mathrm{mg} / \mathrm{L}$ in several wells in the Seminole well field and in one well in the West well field, whereas most wells in the downstream East well field had concentrations $<0.10 \mathrm{mg} / \mathrm{L}$ (fig. 12). In contrast, ammonia- $\mathrm{N}$ and manganese, normally found in greatest abundance in anoxic (reducing) conditions, had the greatest concentrations in the most downstream wells (fig. 12). Ammonia-N concentrations increased from $<0.10 \mathrm{mg} / \mathrm{L}$ in the upstream wells to more than $1.0 \mathrm{mg} / \mathrm{L}$ in the downstream wells. Similarly, manganese concentrations increased from $<100 \mu \mathrm{g} / \mathrm{L}$ upstream to about $1,000 \mu \mathrm{g} / \mathrm{L}$ downstream (fig. 12). The spatial gradient in concentrations of iron was similar to that of manganese.

The observed gradient in water quality in the alluvial aquifer was likely caused by differences in the geomorphology and hydrologic conditions of the upstream and downstream parts of the overlying Cedar River. The upstream reach of the Cedar River is free flowing, whereas the downstream reach is regulated by the five-in-one dam that creates a pool upstream near the East and West well fields (fig. 5). Reaches of the Cedar River that are affected by the regulated section typically have consistent river levels and slower velocity, which reduces the erosive potential of the river thereby increasing deposition of fine-grained material along the streambed and banks. This finegrained material includes organic matter (Siergieiev and others. 2014) and potentially clogs pores in the streambed. Clogging of the pores reduces the velocity of water moving from the river into the alluvial aquifer creating geochemical conditions similar to those previously described in wetlands. Slow movement of water through the streambed and aquifer allow time for biological removal of dissolved oxygen from the water. The anoxic conditions result in reduction of nitrate- $\mathrm{N}$ and dissolution of manganese and iron. Conversely, stream velocity was generally greater in the upstream, unregulated reaches of the river (Siergieiev and others, 2014), which results in increased flushing of fine-grain sediments from the streambed allowing water to more freely move into the adjacent alluvial aquifer. Induced recharge readily transports oxygenated river water along with other dissolved constituents, including nitrate- $\mathrm{N}$, into the aquifer.

The upstream to downstream concentration gradient in nitrate- $\mathrm{N}$ and manganese was interrupted near Seminole vertical production wells Sem15, Sem14, Sem13, and Sem11 (fig. 12). Nitrate- $\mathrm{N}$ concentrations in these wells are substantially less 

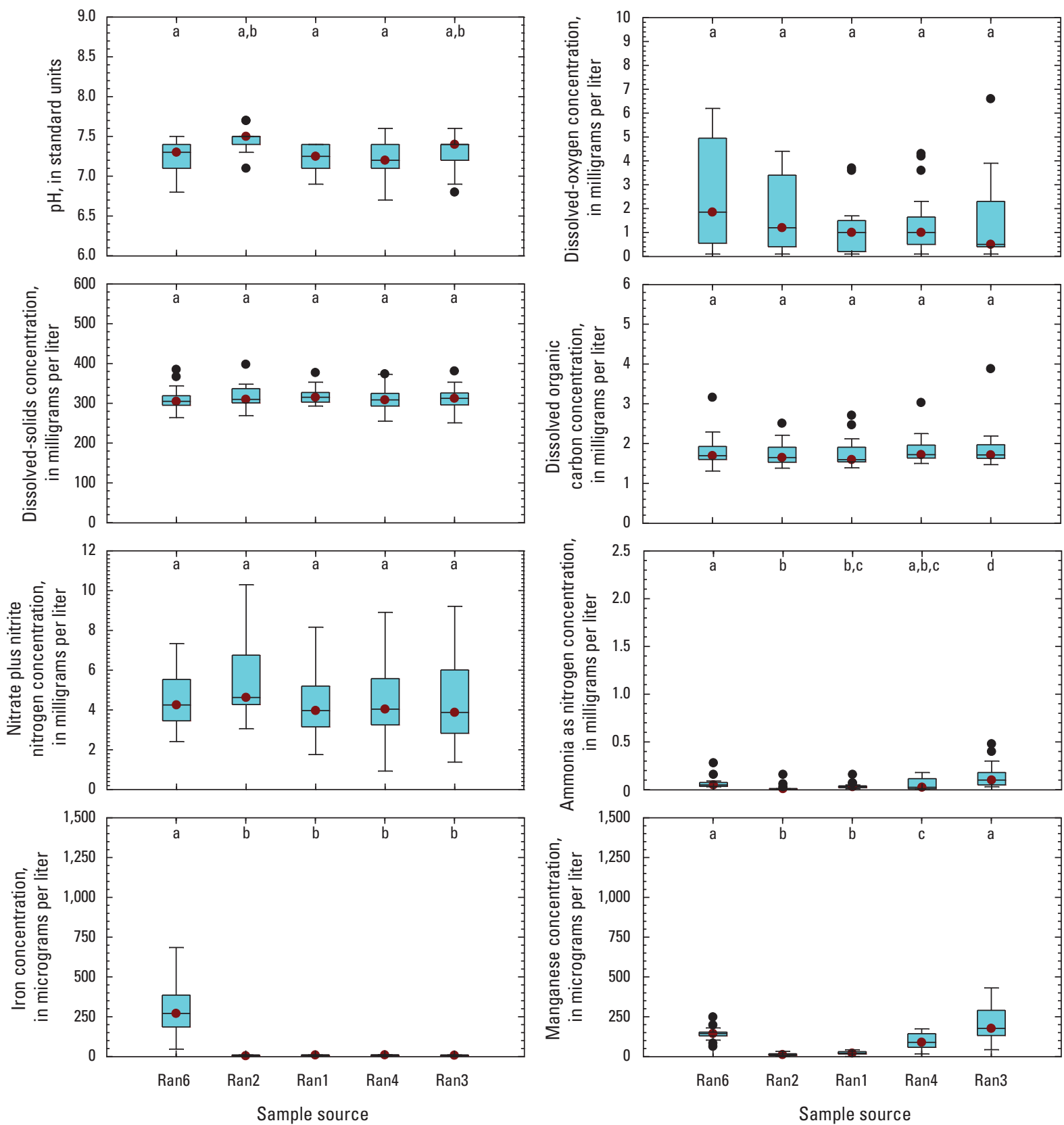

\section{EXPLANATION}

[Data from the U.S. Geological Survey (USGS) National Water Information System database (USGS, 2020); a, b, c, d, and e indicate statistically similar groups. Different letters at the top of each plot indicate statistically significant differences in characteristics between sample sources, whereas same letters indicate sample sources that are not significantly different]

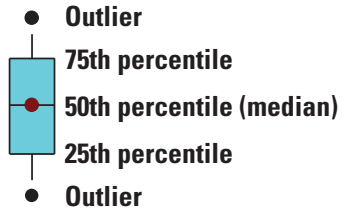

Figure 11. Change in alluvial water quality along a reach of the Cedar River through Cedar Rapids for Ranney production wells in the Seminole well field in Linn County, lowa, 2003-19. 


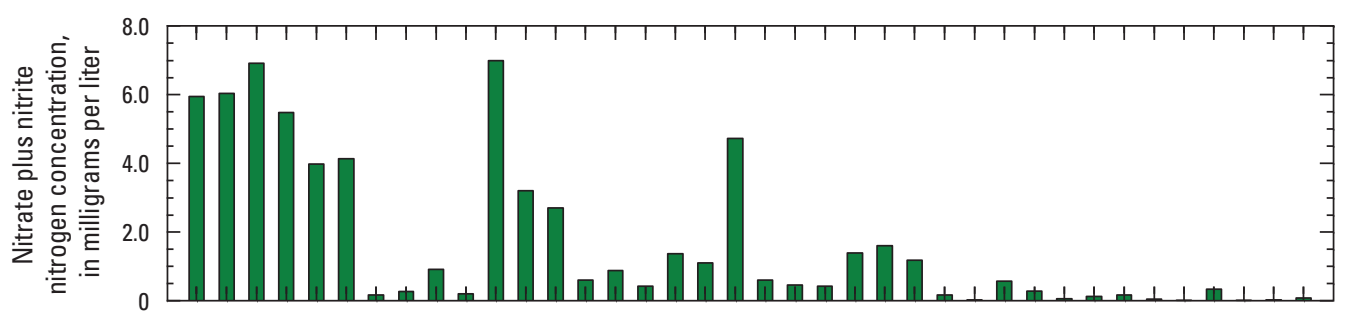

\section{EXPLANATION}

[Data from Jacob Donaghy, Cedar Rapids Utilities

Water Quality Specialist, written commun., 2015]

Secondary maximum contaminant level (U.S. Environmental Protection Agency,

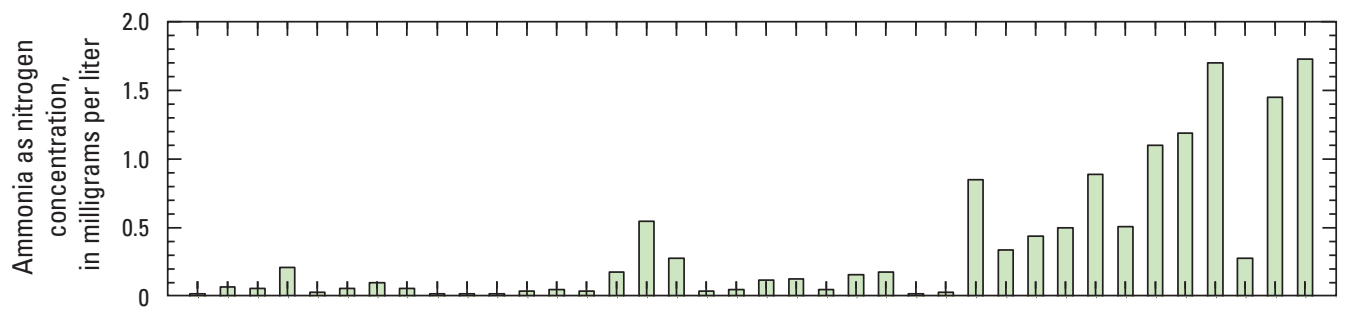

2018)
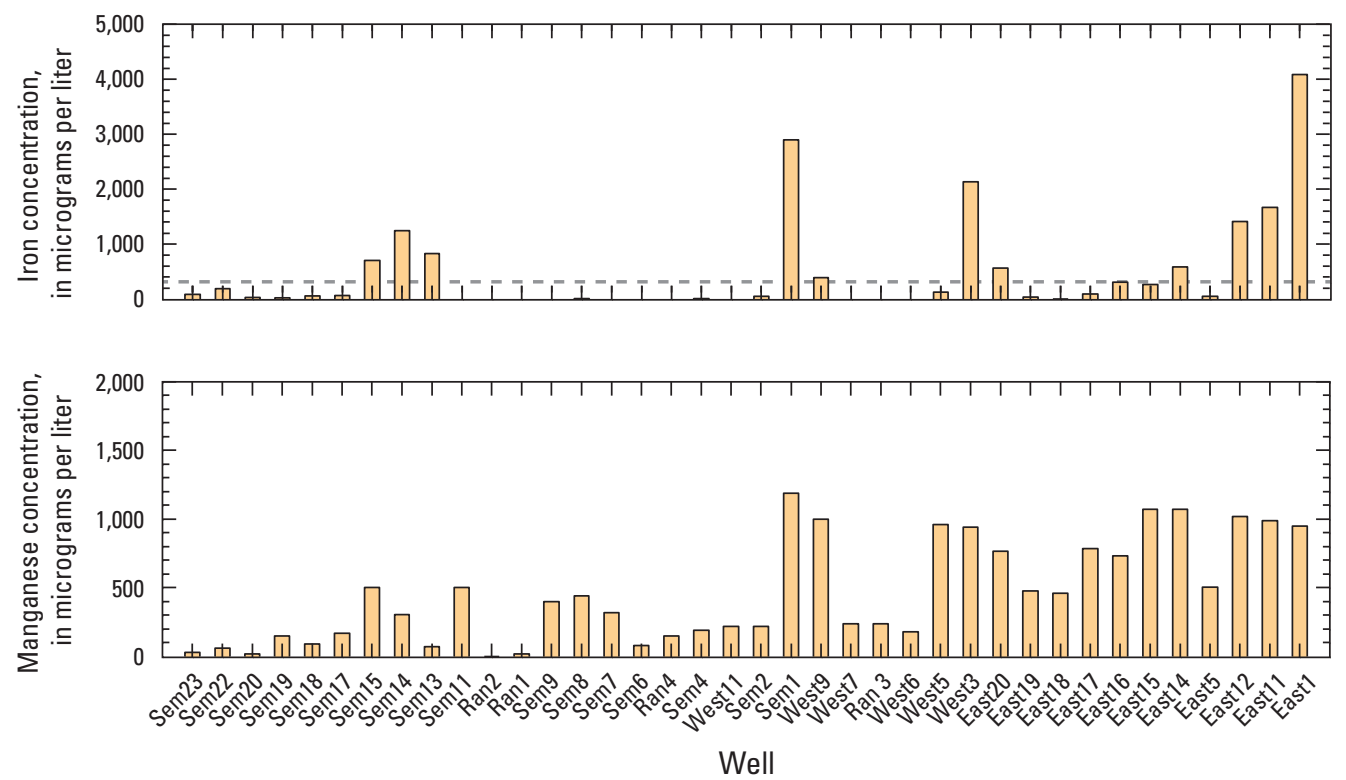

Figure 12. Spatial variability in nitrate plus nitrite nitrogen, ammonia as nitrogen, iron, and manganese concentrations in samples from Cedar Rapids production wells, Linn County, lowa, May 2012, from upstream (left) to downstream (right).

$(<1.0 \mathrm{mg} / \mathrm{L})$ than the other Seminole wells. Manganese concentrations in these wells $(70$ to more than $500 \mu \mathrm{g} / \mathrm{L}$ ) are greater than other nearby Seminole wells $(20-170 \mu \mathrm{g} / \mathrm{L})$. Seminole vertical production wells Sem15, Sem14, Sem13, and Sem11 are near a backwater wetland in contrast to other Seminole wells that are near the Cedar River. Schnoebelen (2008) found that samples from monitoring wells in the alluvial wetland had nitrate- $\mathrm{N}$ concentrations that were four to six times lower when compared to those in the Cedar River. Iron and manganese concentrations in these samples were an order of magnitude greater than compared to samples from the river. Wetlands often have fine-grained sediment with abundant organic matter in their streambed. The fine-grained sediment can impede flow from the wetland to the alluvial aquifer allowing sufficient time for bacteria using organic matter to deplete the dissolved oxygen. In these anoxic conditions, nitrate- $\mathrm{N}$ is reduced to elemental nitrogen by bacterially mediated denitrification (Stumm and Morgan, 1981). Anoxic conditions also are favorable for the dissolution of minerals containing oxidized forms of iron and manganese (Bourg and Bertin, 1993; Schulmeyer and Schnoebelen, 1998).

In addition to differences in hydrologic conditions between upstream and downstream reaches of the Cedar River, differences in soil types and pumping can affect geochemical conditions in the aquifer, which result in variations in redox-affected constituents. The presence of hydric soils enriched with organic carbon can support reducing conditions in shallow underlying aquifers (McMahon and others, 2019). Wetlands and backwater areas containing hydric soils cover a substantial part of the alluvial aquifer in the study area (fig. 1). Schnoebelen (2008) found that anoxic conditions (dissolved oxygen generally $<0.5 \mathrm{mg} / \mathrm{L}$ ) prevailed in the alluvial aquifer in wetland areas, whereas oxic 
conditions (dissolved oxygen concentration $>2.0 \mathrm{mg} / \mathrm{L}$ ) were found in upland areas (Schnoebelen, 2008). The redox conditions resulted in nitrate- $\mathrm{N}$ concentrations that often were four to six times lower in the alluvial aquifer in a wetland area than in the upland area or in the Cedar River. In contrast, iron and manganese concentrations in the wetland areas were an order of magnitude greater when compared to concentrations in the Cedar River or upland well.

Redox conditions do not remain constant in the alluvial aquifer. Changing geochemical conditions caused by hydrological conditions and induced inflow of oxygenated water from the Cedar River can increase nitrate-N concentrations. From 1998 to 2010 , nitrate $-\mathrm{N}$ concentrations ranged from $<0.05$ to $>10.0 \mathrm{mg} / \mathrm{L}$ (the MCL; EPA, 2018) in samples from monitoring well CRM22 (fig. 13). CRM22 is located between the Cedar River and vertical production well Sem4 (fig. 5). CRM22 is less than $100 \mathrm{ft}$ from the Cedar River. Although there were exceptions, nitrate- $\mathrm{N}$ concentrations generally were greatest when dissolved-oxygen concentrations were $>0.5 \mathrm{mg} / \mathrm{L}$ (fig. 13B). Conversely, manganese concentrations were greatest when dissolved-oxygen concentrations were $<1.0 \mathrm{mg} / \mathrm{L}$ (fig. 13B). When substantial dissolved-oxygen concentrations $(>1.0 \mathrm{mg} / \mathrm{L})$ were present in water from CRM22, manganese concentrations were $<50 \mu \mathrm{g} / \mathrm{L}$ and did not exceed the SMCL of $300 \mu \mathrm{g} / \mathrm{L}$. McMahon and others, (2019) speculated that biological degradation of DOC transported from the Cedar River to the alluvial aquifer created anoxic conditions conducive to manganese reduction.

\section{Temporal Variability in Alluvial Water Quality}

Water quality also may vary over time because of factors that affect the quality of the water infiltrating the aquifer from the Cedar River and the underlying Devonian aquifer. During this 30-year study period, temporal changes in climate, land use, and water withdrawals may result in gradual long-term changes in the water quality of the aquifer and in water pumped to supply residential and industrial purposes. These changes may be local within the study area or extend over the entire Cedar River Basin.

Overall, water quality in samples from the Cedar River alluvial aquifer, although variable, did not show sustained changes over the study period. The basic water chemistry of samples collected from the Cedar River alluvial aquifer did not change during the study period (fig. 14). The annual median dissolved-solids concentration varied within a small range from 288 to $345 \mathrm{mg} / \mathrm{L}$ and was normally between 309 and $326 \mathrm{mg} / \mathrm{L}$. DOC was abundant in the aquifer and changed little throughout the study.

Initially in 1993 and 1994, oxidized conditions, characterized by the presence of dissolved oxygen in the water (Meppelink and Kalkhoff, 2021), were prevalent in the aquifer. Reducing conditions were present from 1995 through 2004, after which the dissolved-oxygen concentrations increased and remained constant for the remainder of the study period. The annual median dissolved-oxygen concentration in samples

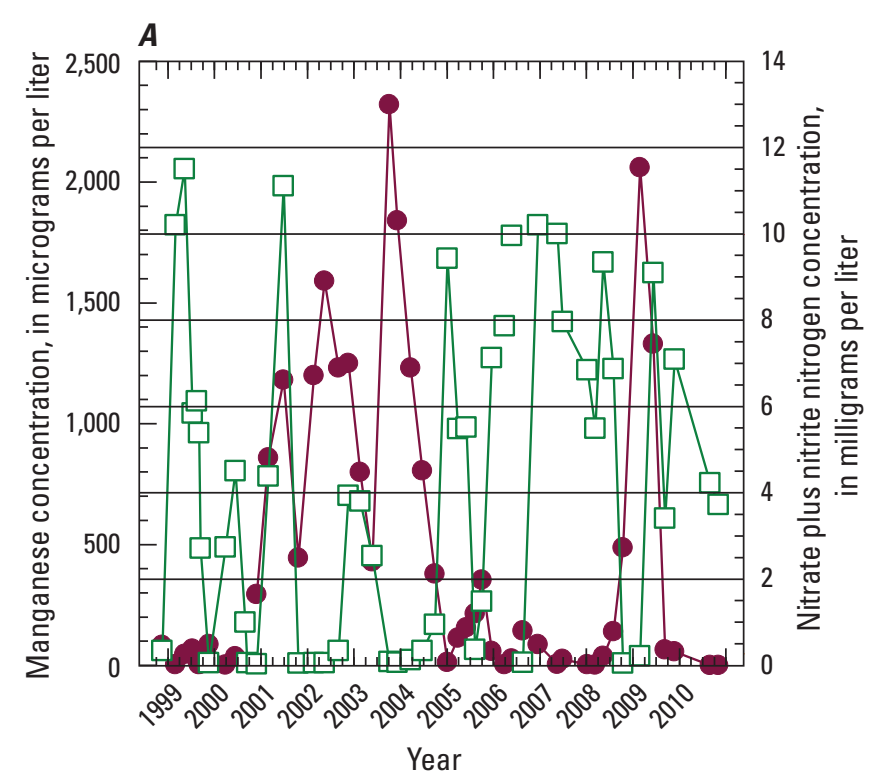

\section{EXPLANATION}

[Data from the U.S. Geological Survey (USGS) National Water Information System database (USGS, 2020)]

- Manganese

$\neg-$ Nitrate plus nitrite nitrogen

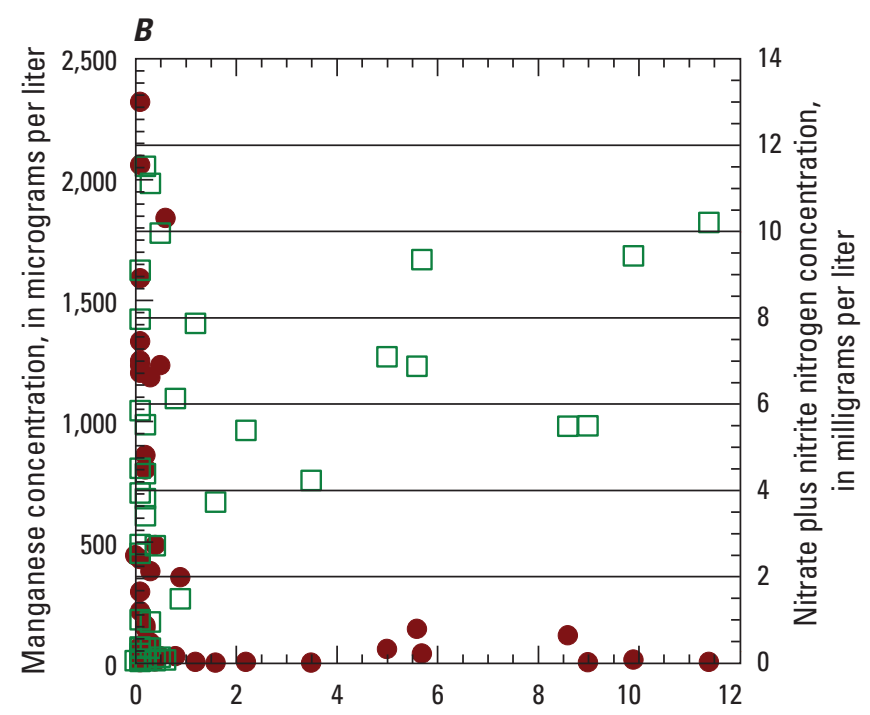

Dissolved-oxygen concentration, in milligrams per liter

\section{EXPLANATION}

[Data from the U.S. Geological Survey (USGS) National Water Information System database (USGS, 2020)]

- Manganese

Nitrate plus nitrite nitrogen

Figure 13. Nitrate plus nitrite nitrogen (nitrate-N) and manganese concentrations in monitoring well CRM22, Linn County, lowa, 1998-2010. A, temporal manganese and nitrate- $\mathrm{N}$ concentrations. $B$, relation between manganese, nitrate- $\mathrm{N}$, and dissolved-oxygen concentrations. 

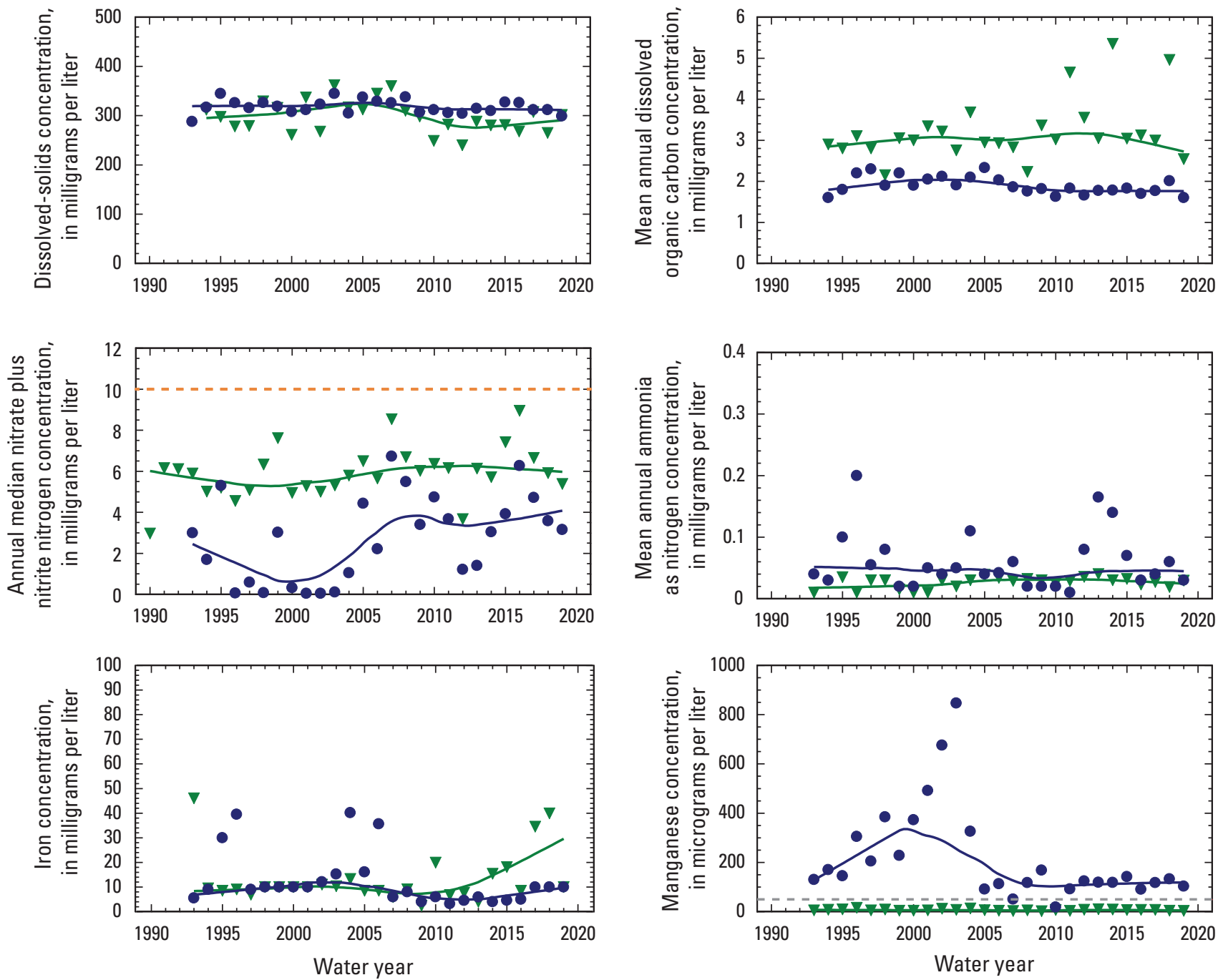

\section{EXPLANATION}

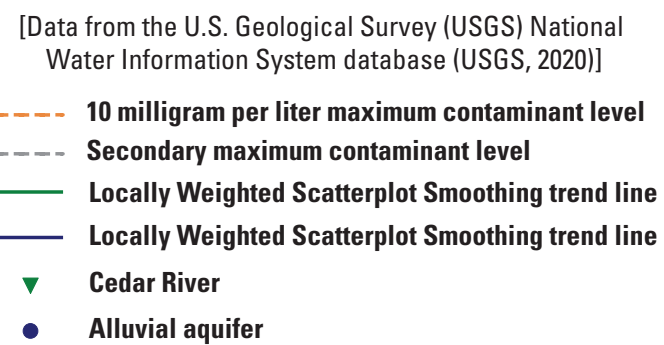

Figure 14. Trends in selected water-quality constituents in the alluvial aquifer and in the Cedar River near Cedar Rapids, Linn County, lowa, 1990-2019.

from 1995 to 2003 was $0.3 \mathrm{mg} / \mathrm{L}$ or less. After 2003, dissolvedoxygen concentrations in samples were generally $>1.0 \mathrm{mg} / \mathrm{L}$, indicating that oxidizing conditions were present in the alluvial aquifer. Reducing conditions (low dissolved-oxygen concentrations) in the alluvial aquifer in 1995-2003 were associated with low-flow streamflow in the Cedar River during the same period (fig. 2).
Annual median concentrations of the oxidized constituents such as nitrate- $\mathrm{N}$ were greater during years when the annual median dissolved-oxygen concentrations were greater, and annual median concentrations of reduced compounds such as manganese and iron were greater during years when dissolvedoxygen concentrations were lower (fig. 14). Nitrate-N concentrations varied greatly in the alluvial aquifer as previously discussed. Even with the large variability, there was a statistically 
significant $(p<0.05)$ linear increase in nitrate- $\mathrm{N}$ concentrations in samples collected from the alluvial aquifer during the study period.

Annual concentrations of two commonly detected pesticides, atrazine and acetochlor, decreased in the Cedar River alluvial aquifer during the study period. The annual mean atrazine concentration in all alluvial aquifer samples decreased linearly from $0.54 \mu \mathrm{g} / \mathrm{L}$ in 1993 to about $0.10 \mu \mathrm{g} / \mathrm{L}$ in 2019 . Annual mean acetochlor concentrations remained relatively constant in the range from 0.04 to $0.06 \mu \mathrm{g} / \mathrm{L}$ until 2015 when the annual mean concentrations decreased to $0.03 \mu \mathrm{g} / \mathrm{L}$ or less (fig. 15). The degradation compound, CIET, followed its parent compound, atrazine, annual mean concentration trend during the study period (fig. 15).

It was notable that while annual mean atrazine and acetochlor concentrations in the alluvial aquifer were decreasing linearly, concentrations in the Cedar River exhibited a U-shaped trend; concentrations decreased from the early 1990s to the mid-2000s before again increasing through the end of the study period (fig. 15). Pesticide use data are not available for the Cedar River Basin, but the national trend shows use of atrazine, acetochlor, and metolachlor decreased from the 1990s to the mid-2000s and then increased through the 2010s (USGS, 2019).
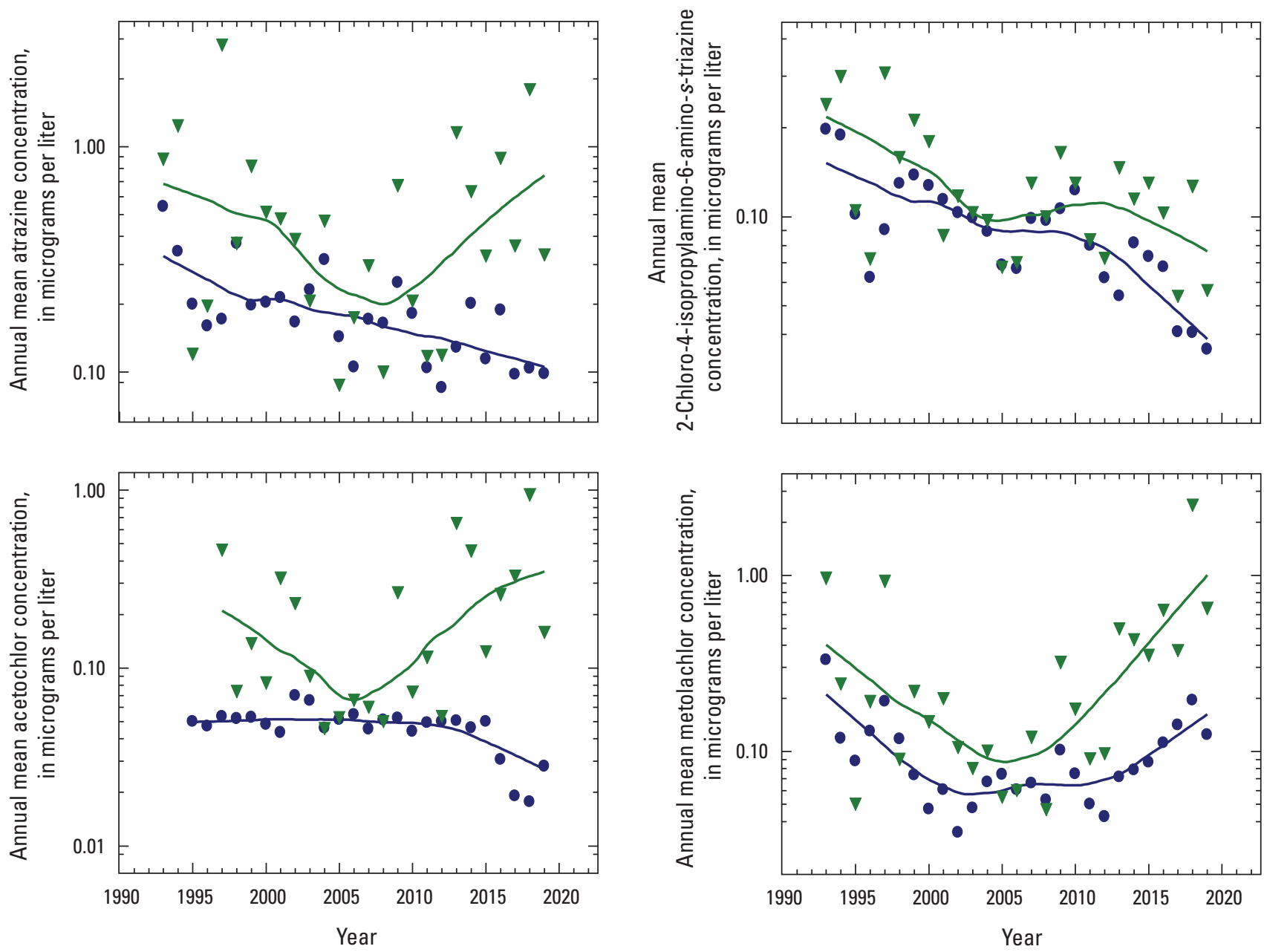

\section{EXPLANATION}

[Data from the U.S. Geological Survey (USGS) National Water Information System database (USGS, 2020); all samples from alluvial wells used for calculation of annual mean]

$\begin{array}{ll}- & \text { Locally Weighted Scatterplot Smoothing trend line } \\ - & \text { Locally Weighted Scatterplot Smoothing trend line } \\ \nabla & \text { Cedar River } \\ - & \text { Alluvial aquifer }\end{array}$

Figure 15. Trend in concentrations of the four most commonly detected pesticide compounds in the Cedar River alluvial aquifer and the Cedar River in Linn County, lowa, 1990-2019. 
The similar temporal pattern between pesticide use and annual mean concentrations in the Cedar River indicates concentrations in the Cedar River Basin are likely caused by pesticide use in the basin, but reason for the divergence in atrazine and acetochlor trends in 2005 is unknown.

Annual metolachlor concentrations more closely followed those in the Cedar River. Annual mean metolachlor concentrations exhibited a U-shaped trend in the Cedar River and in the alluvial aquifer. Use of metolachlor nationwide decreased by almost 50 percent from the 1990s to the mid-2000s (USGS, 2019). The decrease may have been largely because of the change in formulation in the late 1990s. After reaching a low in the mid-2000s, metolachlor use in the United States doubled by the end of the study period (USGS, 2019). Assuming use in the Cedar River Basin mirrored national use, changes in pesticide application could account for the trend in metolachlor concentrations in the Cedar River alluvial aquifer and the Cedar River.

\section{Water Quality in Source Waters}

The Cedar River alluvial aquifer within the study area is supplied by three main sources of water: direct recharge from precipitation, the Devonian aquifer, and the Cedar River (Boyd, 1998; Schulmeyer and Schnoebelen, 1998; Turco and Buchmiller, 2004;). The chemical composition of the bedrock and the quality of the river water alters the water quality of the alluvial aquifer because of mixing and alteration of geochemical conditions in the aquifer. Temporal changes in the water quality in the Devonian aquifer and the Cedar River can result in similar changes in the alluvial aquifer near Cedar Rapids, Iowa.

\section{Surficial Recharge}

Water in the alluvial aquifer is recharged primarily from infiltration of rainfall with some water originating from the adjacent uplands consisting of glacial till and loess deposits (Boyd, 1999). Monitoring of the alluvial aquifer in a forested area near the margin of the alluvial aquifer (monitoring well CRM15) and near an area with agricultural fields and other mixed land uses (monitoring well CRM25) indicated that the surficial recharge was a calcium bicarbonate type. Sulfate and silica are present in smaller concentrations, and the shallow groundwater, indicative of the surficial recharge, was highly oxygenated (table 9). Boyd (1999) describes the water chemistry in recharge areas of the alluvial aquifer a result of carbonate equilibrium and aluminosilicate weathering reactions that occur as precipitation infiltrates into the groundwater. Shallow groundwater from CRM25 located in a recharge area near agricultural fields has elevated nitrate- $\mathrm{N}$ and chloride concentrations in relation to recharge area in forested areas (table 9). Pesticides commonly used on agricultural crops were present more often and at greater concentrations in shallow groundwater in agricultural recharge areas (table 10) than in forested areas. Atrazine and one of its degradation compounds, CIAT, were present in all samples but in concentrations less than drinking-water standard (EPA, 2018) of $3.0 \mu \mathrm{g} / \mathrm{L}$ (table 10). Another atrazine degradation compound, CEAT, was found in about 33 percent of all samples in small concentrations.

Except for the metolachlor degradate, metolachlor ethanesulfonic acid, pesticides were not detected in the recharge area of the alluvial aquifer near monitoring well CRM15 (Boyd, 2000). Land was undeveloped near CRM15 and was surrounded by prairie and wooded wetlands; thus, little application of herbicides would be expected near the well. A possible source of metolachlor ethanesulfonic acid may be from floodwater infiltrating through soil, but pesticide compounds may also originate directly from rainfall. Several commonly used herbicides and insecticides are transported locally and regionally in the atmosphere (Foreman and others, 2000; Peck and Hornbuckle, 2005) and are deposited in rainfall in the Cedar Rapids area (Majewski and others, 2000).

\section{Devonian Aquifer}

The basic water chemistry in the Devonian aquifer near Cedar Rapids was substantially different than that in the overlying alluvial aquifer. The water was more dilute with significantly ( $p<0.05$, Kruskal-Wallis) less dissolved solids than in alluvial water. Calcium and magnesium are the predominant cations in solution. Magnesium concentrations are greater than in water from the shallower part of the aquifer because of dissolution from dolomite within the Devonian-aged formations (Hansen, 1970). Bicarbonate was present to the exclusion of other anions. Calcium, sodium, chloride, and sulfate concentrations are significantly lower in the Devonian aquifer than in the alluvial aquifer. Concentrations of nitrate- $\mathrm{N}$ and orthophosphate, constituents commonly associated with agricultural and urban runoff, were significantly smaller in the Devonian aquifer than in the alluvial aquifer.

Total dissolved-solids concentrations normally ranged from 238 to $345 \mathrm{mg} / \mathrm{L}$ but were as great as $600 \mathrm{mg} / \mathrm{L}$ (table 11). The water had a generally neutral $\mathrm{pH}$ most often in a small range from about 7.2 to 7.6 standard units. The water was anoxic; dissolved-oxygen concentrations were generally $<0.5 \mathrm{mg} / \mathrm{L}$ (75th percentile). DOC was present in appreciable amounts that ranged from less than 0.20 to $2.90 \mathrm{mg} / \mathrm{L}$. Nitrogen and phosphorus concentrations were small; nitrate- $\mathrm{N}$ normally ranged from less than detection to $0.08 \mathrm{mg} / \mathrm{L}$, and orthophosphate concentration normally was $0.02 \mathrm{mg} / \mathrm{L}$ (75 percentile) or less (table 11).

The anoxic/reducing conditions with little or no nitrate- $\mathrm{N}$ facilitates a reducing environment in the Devonian aquifer that creates conditions favorable for dissolution of iron and manganese from aquifer materials. Iron concentrations normally ranged from 7.00 to $220 \mu \mathrm{g} / \mathrm{L}$, and manganese concentrations ranged from 5.00 to $612 \mu \mathrm{g} / \mathrm{L}$. Iron concentrations are generally smaller than those reported by Wahl and Bunker (1986) in samples collected from the Devonian aquifer in Linn and 
Table 9. Summary of the physical properties, major ions, nutrients, and trace elements in forested (monitoring well CRM15) and agricultural (monitoring well CRM25) recharge areas of the alluvial aquifer near Cedar Rapids, Linn County, lowa, $1996-2003$.

[Data are summarized from the U.S. Geological Survey (USGS) National Water Information System database (USGS, 2020); search using the parameter code to see full parameter names, descriptions, and other data in the database. $n$, number of samples; $\mathrm{mg} / \mathrm{L}$, milligram per liter; $\mu \mathrm{S} / \mathrm{cm}$ at $25^{\circ} \mathrm{C}$, microsiemens per centimeter at 25 degrees Celsius; ${ }^{\circ} \mathrm{C}$, degree Celsius; --, not applicable; $\mathrm{SiO}_{2}$, silicon dioxide; $\mathrm{NH}_{4}^{+}$, ammonium; $\mathrm{N}$, nitrogen; $\mathrm{P}$, phosphorus; $\mu \mathrm{g} / \mathrm{L}$, microgram per liter]

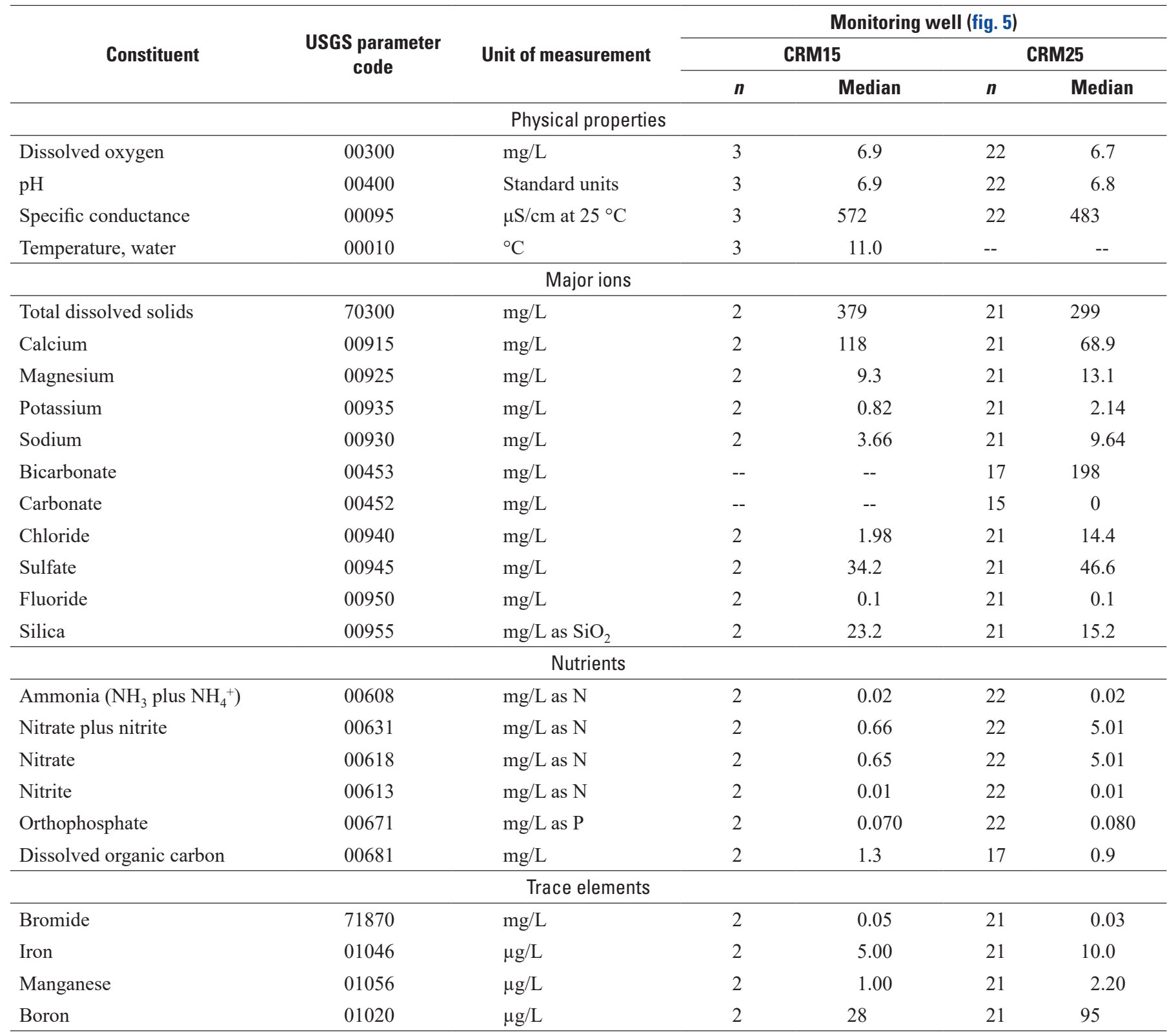


Table 10. Occurrence of selected pesticides in forested (monitoring well CRM15) and agricultural (monitoring well CRM25) recharge areas of the Cedar River alluvial aquifer in Linn County, lowa, 1996-2003.

[Data are summarized from the U.S. Geological Survey (USGS) National Water Information System database (USGS, 2020); search using the parameter code to see full parameter names, descriptions, and other data in the database. $n$, number of samples; $\%$, percent; $\mu \mathrm{g} / \mathrm{L}$, microgram per liter; <, less than minimum reporting level indicated; E, detected but estimated concentration less than minimum reporting level]

\begin{tabular}{|c|c|c|c|c|c|}
\hline \multirow[b]{3}{*}{ Pesticide compound } & \multirow[b]{3}{*}{ USGS parameter code } & \multicolumn{4}{|c|}{ Monitoring well (fig. 5) } \\
\hline & & \multicolumn{2}{|c|}{ CRM15 (n=5) } & \multicolumn{2}{|c|}{ CRM25 (n=21) } \\
\hline & & $\begin{array}{l}\text { Detection } \\
\text { rate } \\
(\%)\end{array}$ & $\begin{array}{c}\text { Maximum } \\
\text { concentration } \\
(\mu \mathrm{g} / \mathrm{L})\end{array}$ & $\begin{array}{c}\text { Detection } \\
\text { rate } \\
(\%)\end{array}$ & $\begin{array}{c}\text { Maximum } \\
\text { concentration } \\
(\mu \mathrm{g} / \mathrm{L})\end{array}$ \\
\hline $\begin{array}{l}\text { 2-Chloro-4-isopropylamino-6-amino- } \\
s \text {-triazine (CIAT) }\end{array}$ & 04040 & 0.00 & $<0.05$ & 100 & 0.50 \\
\hline Atrazine & 39632 & 0.00 & $<0.05$ & 100 & 1.34 \\
\hline $\begin{array}{l}\text { 2-Chloro-6-ethylamino-4-amino-s- } \\
\text { triazine (CEAT) }\end{array}$ & 04038 & 0.00 & $<0.05$ & 33.0 & 0.10 \\
\hline Prometon & 04037 & 0.20 & E0.04 & 5.00 & E0.01 \\
\hline Metribuzin & 82630 & 0.00 & $<0.05$ & 14.0 & E0.04 \\
\hline Metolachlor & 39415 & 0.00 & $<0.05$ & 5.00 & 0.21 \\
\hline Simazine & 04035 & 0.00 & $<0.05$ & 5.00 & E0.02 \\
\hline Dicrotophos & 38535 & 0.00 & $<0.05$ & 5.00 & E0.02 \\
\hline Acetochlor & 49260 & 0.00 & $<0.05$ & 5.00 & 0.06 \\
\hline Trifluralin & 04023 & 0.00 & $<0.05$ & 0.00 & $<0.05$ \\
\hline Propachlor & 04024 & 0.00 & $<0.05$ & 0.00 & $<0.05$ \\
\hline Hexazinone & 04025 & 0.00 & $<0.05$ & 0.00 & $<0.05$ \\
\hline Butachlor & 04026 & 0.00 & $<0.05$ & 0.00 & $<0.05$ \\
\hline Carboxin & 04027 & 0.00 & $<0.05$ & 0.00 & $<0.05$ \\
\hline Butylate & 04028 & 0.00 & $<0.05$ & 0.00 & $<0.05$ \\
\hline Bromacil & 04029 & 0.00 & $<0.05$ & 0.00 & $<1.00$ \\
\hline Simetryn & 04030 & 0.00 & $<0.05$ & 0.00 & $<0.05$ \\
\hline Cycloate & 04031 & 0.00 & $<0.05$ & 0.00 & $<0.05$ \\
\hline Terbacil & 04032 & 0.00 & $<0.05$ & 0.00 & $<0.05$ \\
\hline Diphenamid & 04033 & 0.00 & $<0.05$ & 0.00 & $<0.05$ \\
\hline Vernolate & 04034 & 0.00 & $<0.05$ & 0.00 & $<0.05$ \\
\hline Prometryn & 04036 & 0.00 & $<0.05$ & 0.00 & $<0.05$ \\
\hline Cyanazine & 04041 & 0.00 & $<0.20$ & 0.00 & $<0.20$ \\
\hline Ametryn & 38401 & 0.00 & $<0.05$ & 0.00 & $<0.05$ \\
\hline Alachlor & 46342 & 0.00 & $<0.05$ & 0.00 & $<0.05$ \\
\hline
\end{tabular}


Table 11. Summary of the physical properties, major ions, nutrients, and trace elements in the Devonian aquifer near Cedar Rapids, Linn County, lowa, 1990-2019.

[Data are summarized from the U.S. Geological Survey (USGS) National Water Information System database (USGS, 2020); search using the parameter code to see full parameter names, descriptions, and other data in the database. $n$, number of samples; $\mathrm{mg} / \mathrm{L}$, milligram per liter; $<$, less than; $\mu \mathrm{S} / \mathrm{cm}$ at $25^{\circ} \mathrm{C}$, microsiemens per centimeter at 25 degrees Celsius; ${ }^{\circ} \mathrm{C}$, degree Celsius; $\mathrm{SiO}_{2}$, silicon dioxide; $\mathrm{NH}_{4}{ }^{+}$, ammonium; $\mathrm{N}$, nitrogen; $\mathrm{P}$, phosphorus; $\mu \mathrm{g} / \mathrm{L}$, microgram per liter]

\begin{tabular}{|c|c|c|c|c|c|c|c|c|c|}
\hline \multirow[b]{2}{*}{ Constituent } & \multirow{2}{*}{$\begin{array}{c}\text { USGS } \\
\text { parameter } \\
\text { code }\end{array}$} & \multirow{2}{*}{$\begin{array}{c}\text { Unit of } \\
\text { measurement }\end{array}$} & \multirow[b]{2}{*}{$n$} & \multicolumn{6}{|c|}{ Summary statistics } \\
\hline & & & & Minimum & $\begin{array}{c}\text { 25th } \\
\text { percentile }\end{array}$ & Median & Mean & $\begin{array}{c}\text { 75th } \\
\text { percentile }\end{array}$ & Maximum \\
\hline Dissolved oxygen & 00300 & $\mathrm{mg} / \mathrm{L}$ & 39 & $<0.10$ & 0.10 & 0.20 & 0.81 & 0.50 & 5.70 \\
\hline $\mathrm{pH}$ & 00400 & Standard units & 39 & 6.9 & 7.2 & 7.4 & 7.5 & 7.6 & 8.8 \\
\hline Temperature, water & 00010 & ${ }^{\circ} \mathrm{C}$ & 40 & 6.8 & 10.0 & 10.8 & 10.8 & 11.8 & 15.0 \\
\hline \multicolumn{10}{|c|}{ Major ions } \\
\hline $\begin{array}{l}\text { Total dissolved } \\
\text { solids }\end{array}$ & 70300 & $\mathrm{mg} / \mathrm{L}$ & 33 & 152 & 238 & 283 & 301 & 345 & 600 \\
\hline Sodium & 00930 & $\mathrm{mg} / \mathrm{L}$ & 33 & 3.71 & 4.23 & 9.30 & 10.1 & 11.0 & 50.0 \\
\hline Bicarbonate & 00453 & $\mathrm{mg} / \mathrm{L}$ & 17 & 200 & 289 & 295 & 308 & 344 & 389 \\
\hline Carbonate & 00452 & $\mathrm{mg} / \mathrm{L}$ & 17 & $<0.1$ & $<0.1$ & $<0.1$ & 0.12 & 0.20 & 0.50 \\
\hline Chloride & 00940 & $\mathrm{mg} / \mathrm{L}$ & 33 & 0.45 & 0.57 & 6.30 & 13.7 & 20.0 & 67.0 \\
\hline Sulfate & 00945 & $\mathrm{mg} / \mathrm{L}$ & 33 & 2.80 & 7.11 & 11.2 & 17.7 & 21.0 & 93.0 \\
\hline Fluoride & 00950 & $\mathrm{mg} / \mathrm{L}$ & 33 & 0.10 & 0.20 & 0.28 & 0.25 & 0.30 & 0.40 \\
\hline Silica & 00955 & $\mathrm{mg} / \mathrm{L}$ as $\mathrm{SiO}_{2}$ & 33 & 9.00 & 13.0 & 13.9 & 14.8 & 17.4 & 24.0 \\
\hline \multicolumn{10}{|c|}{ Nutrients } \\
\hline $\begin{array}{c}\text { Ammonia }\left(\mathrm{NH}_{3}\right. \\
\left.\text { plus } \mathrm{NH}_{4}^{+}\right)\end{array}$ & 00608 & $\mathrm{mg} / \mathrm{L}$ as $\mathrm{N}$ & 28 & 0.01 & 0.22 & 0.28 & 0.99 & 1.70 & 3.80 \\
\hline \multicolumn{10}{|c|}{ Trace elements } \\
\hline Bromide & 71870 & $\mathrm{mg} / \mathrm{L}$ & 33 & 0.01 & 0.02 & 0.03 & 0.03 & 0.04 & 0.10 \\
\hline Iron & 01046 & $\mu \mathrm{g} / \mathrm{L}$ & 33 & 3.00 & 7.00 & 28.0 & 646 & 220 & 3,900 \\
\hline Manganese & 01056 & $\mu \mathrm{g} / \mathrm{L}$ & 33 & 3.00 & 5.00 & 54.0 & 388 & 612 & 2,200 \\
\hline Boron & 01020 & $\mu \mathrm{g} / \mathrm{L}$ & 29 & 21 & 40 & 43 & 54 & 50 & 260 \\
\hline
\end{tabular}


Johnson Counties in the 1960-70s. Wahl and Bunker (1986) found that the concentrations of iron were relatively high, generally from 350 to $6,500 \mu \mathrm{g} / \mathrm{L}$, and manganese concentrations were $<100 \mu \mathrm{g} / \mathrm{L}$. The highest manganese concentrations occurred in wells with the greatest iron concentrations.

Few pesticide compounds were detected in the Devonian aquifer and, when present, were detected in small concentrations (table 7). Five pesticides were detected, the most common being atrazine (about 20 percent of the samples). Metolachlor was the second most frequently detected (about 17 percent) compound. The maximum concentration of any pesticide was $<0.1 \mu \mathrm{g} / \mathrm{L}$.

Water quality in the Devonian aquifer remained relatively constant over time. Initial seasonal sampling of the Devonian monitoring wells in the early 1990s showed that water quality was relatively consistent through the year. Subsequently, these wells were sampled on an annual basis resulting in relatively few samples through the study period. Because of the small sample size, a statistical trend test was not completed; however, visual analysis (fig. 16) of the samples over time indicated that the physical properties and the concentrations of most major ions, nutrients, and selected trace elements remained relatively constant through the study period. An example was the analytical results of samples from monitoring well CRM6 and its replacement well, CRM6A (fig. 16).

Dissolved-solids concentrations mostly remained between 200 and $300 \mathrm{mg} / \mathrm{L}$, nitrate $-\mathrm{N}$ concentrations mostly remained at $0.05 \mathrm{mg} / \mathrm{L}$ or less, and ammonia $-\mathrm{N}$ concentrations remained between 0.26 and $0.33 \mathrm{mg} / \mathrm{L}$. Iron and manganese concentrations were constant except for increased iron concentrations during the last 2 years from $<30 \mu \mathrm{g} / \mathrm{L}$ to $>80 \mu \mathrm{g} / \mathrm{L}$. The water quality in the Devonian aquifer probably remains constant because it was substantially older than water in the alluvial aquifer (Boyd, 1998) and was not affected by short-term climatic and land use changes that would affect the water chemistry.

Though water quality in the Devonian aquifer varied little over time, there was spatial variation across the study area. Water quality at three wells, one from each well field, were compared to explore these spatial variations (table 12). Although only three to nine samples are available, depending on the constituent and well (table 12), the data indicate waterquality changes from upstream in the Devonian aquifer at the Seminole well field to downstream at the West and East well
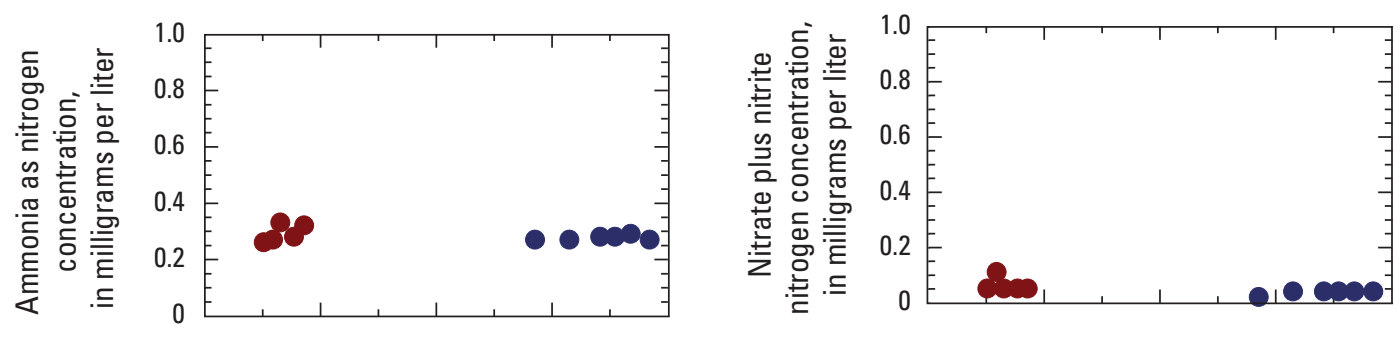

\section{EXPLANATION}

[Data from the U.S. Geological Survey (USGS) National Water Information System database (USGS, 2020)]

\section{Monitoring well CRM6}

- Monitoring well CRM6A
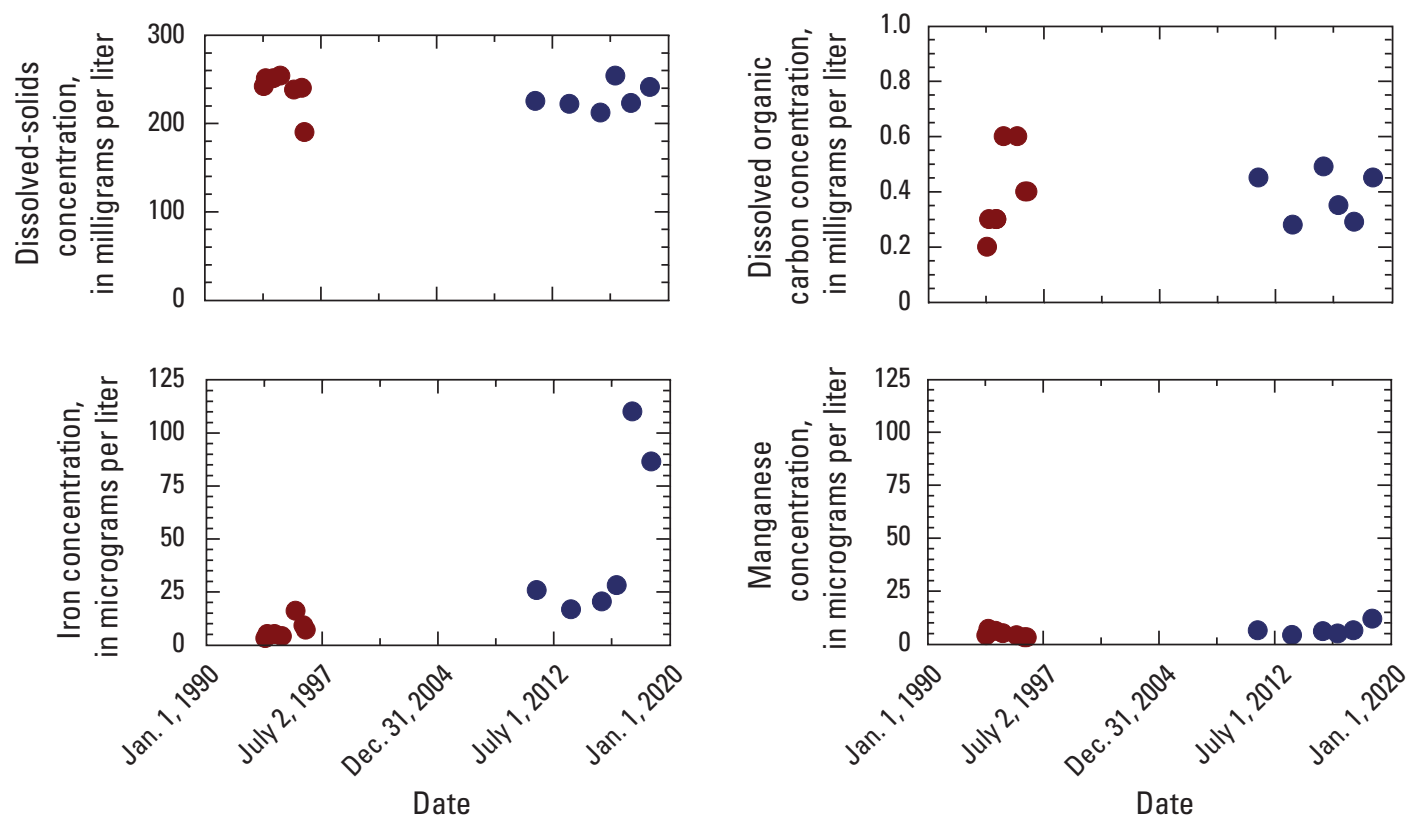

Figure 16. Temporal trend in water quality in the Devonian aquifer Cedar Rapids Seminole well field (monitoring wells CRM6 and CRM6A), Linn County, lowa, 1990-2019. 
fields. Dissolved-solids concentrations were lowest in samples from the upstream monitoring well CRM-6, and highest at the downstream monitoring wells CRM-7 and CRM-12. Median sulfate concentrations were significantly greater $(p<0.05$, Kruskal-Wallis test) in monitoring well CRM-7 in the West well field than in the Seminole and East well fields. There was no significant ( $p>0.05$, Kruskal-Wallis test) spatial difference in the median nitrate- $\mathrm{N}$ concentration in samples from monitoring wells in the Devonian aquifer.

\section{Cedar River}

The water in the Cedar River was a composite of runoff from all point and nonpoint sources and seepage from groundwater in the 6,342- $\mathrm{mi}^{2}$ basin upstream from the study area. Water quality was heavily affected by agriculture, which contributes to excess nitrogen, phosphorus, and pesticides in runoff. Additional nitrogen and phosphorus may be generated by point-source discharges from cities and towns in the basin and runoff from urban or suburban lands.

The Cedar River near Cedar Rapids was a welloxygenated environment with a neutral to basic $\mathrm{pH}$. The median dissolved-oxygen concentration was $11.5 \mathrm{mg} / \mathrm{L}$, and the median $\mathrm{pH}$ was 8.2 standard units (table 13). Only one sample had a dissolved-oxygen concentration $<5.0 \mathrm{mg} / \mathrm{L}$. Dissolved-oxygen concentrations and $\mathrm{pH}$ values in the Cedar River were significantly ( $p<0.05$, Kruskall Wallis test) greater than in the adjacent alluvial aquifer and underlying Devonian bedrock aquifers (fig. 9).

The water in the Cedar River was a calcium, magnesium, bicarbonate type. Total dissolved-solids concentrations ranged from 154 to $470 \mathrm{mg} / \mathrm{L}$ (table 13) during the 1990-2019 study and normally (25th to 75th percentile) ranged from 261 to $342 \mathrm{mg} / \mathrm{L}$. A large part of the dissolved solids consisted of calcium (mean of $62.6 \mathrm{mg} / \mathrm{L}$ ) and bicarbonate (mean of $206 \mathrm{mg} / \mathrm{L}$ ). Magnesium (mean of $20.1 \mathrm{mg} / \mathrm{L}$ ), chloride (mean of $22.4 \mathrm{mg} / \mathrm{L}$ ), and sulfate (mean of $29.0 \mathrm{mg} / \mathrm{L}$ ) were a smaller part of the dissolved solids.

Nitrate-N concentrations in the Cedar River were variable during the study period and ranged from less than detection to $12.6 \mathrm{mg} / \mathrm{L}$. Nitrate-N concentrations normally ranged from 3.48 to $7.30 \mathrm{mg} / \mathrm{L}$ (table 13), but 8.9 percent exceeded the $10.0 \mathrm{mg} / \mathrm{L}$ MCL (EPA, 2018). Nitrate-N concentrations in the river varied seasonally and are greatest during the spring and summer growing seasons when streamflow was generally the greatest. Exceedances of the nitrate-N MCL occurred during these two seasons. Nitrate-N concentrations were significantly ( $p<0.05$, Kruskall-Wallis) smaller in the fall at the end of the growing season when streamflow was generally the smallest, and dissolved-oxygen concentrations were commonly $>10 \mathrm{mg} / \mathrm{L}$. Low streamflow in the fall with its associated stream clarity was conducive to growth of planktonic and benthic algae. Production by algae can contribute to the higher dissolved-oxygen concentrations in the Cedar River during the fall.
Ammonia- $\mathrm{N}$, which is readily transformed to nitrite and nitrate and incorporated into aquatic plants and algae, was present in low concentrations in the Cedar River. Ammonia-N concentrations were less than $<0.04 \mathrm{mg} / \mathrm{L}$ in 75 percent of the samples (table 13). Overall, ammonia-N concentrations in the Cedar River were not significantly different $(p>0.05)$ than those in the alluvial aquifer but were less than those in the Devonian aquifer (fig. 9).

Iron and manganese, two trace elements of concern to water suppliers, were present in low concentrations in the Cedar River through the study period (table 13). Iron concentrations were normally from 7.00 to $18.1 \mu \mathrm{g} / \mathrm{L}$. The maximum concentration of $1,300 \mu \mathrm{g} / \mathrm{L}$ was the only sample exceeding the EPA SMCL of $300 \mu \mathrm{g} / \mathrm{L}$ (EPA, 2018) for iron. Manganese concentrations were also small normally ranging from 3.66 to $10.0 \mu \mathrm{g} / \mathrm{L}$. Three samples had concentrations that exceeded the $50 \mu \mathrm{g} / \mathrm{L}$ secondary drinking-water standard (EPA, 2018). The well oxygenated and neutral to basic $\mathrm{pH}$ of water in the Cedar River are conditions where dissolved iron and manganese precipitates out of solution. The small iron and manganese concentrations in the Cedar River contrast with those in the alluvial and Devonian aquifers. Median iron concentrations from Cedar River samples were significantly $(p<0.05$, Kruskall-Wallis) smaller than in samples from vertical production wells. Median iron concentrations in samples from the Cedar River are not significantly ( $p>0.05$, Kruskall-Wallis) different than those from the Ranney production wells and monitoring wells. The difference in manganese concentrations between the river and the alluvial and Devonian aquifers was more distinct than that for iron (fig. 9). Whereas the median manganese concentration in the Cedar River was $6.43 \mu \mathrm{g} / \mathrm{L}$, the median manganese concentration was $150 \mu \mathrm{g} / \mathrm{L}$ in the alluvial aquifer (table 6) and was $54 \mu \mathrm{g} / \mathrm{L}$ in the Devonian aquifer (table 11).

DOC was present in appreciable quantities in the Cedar River. DOC concentrations were normally from 2.60 to $3.86 \mathrm{mg} / \mathrm{L}$ and were significantly $(p<0.05)$ greater than in the alluvial and Devonian aquifers (fig. 9, table 13).

Twenty-four synthetic organic pesticide compounds were detected in at least one sample from the Cedar River during the study period (table 7). Several triazine and acetanolide herbicides were the most detected synthetic organic compounds in the Cedar River. Atrazine and its degradation product (CIAT) were detected in 99.2 and 96.7 percent of the samples, respectively. The atrazine concentration occasionally (about 4 percent of the samples) exceeded the MCL of $3.0 \mu \mathrm{g} / \mathrm{L}$ (EPA, 2018), and the maximum concentration $(8.16 \mu \mathrm{g} / \mathrm{L})$ was more than twice the standard. The maximum concentration of CIAT $(0.66 \mu \mathrm{g} / \mathrm{L})$ was much smaller than that of its parent compound. The acetanolide herbicide, metolachlor, was also detected in more than 90 percent of the samples. The maximum metolachlor concentration was $9.67 \mu \mathrm{g} / \mathrm{L}$. A second acetanolide herbicide, acetochlor, was the only other synthetic organic compound detected in $>50$ percent of the samples (table 7). Commercial sales of alachlor and cyanazine were discontinued during the study period, which probably limited 
Table 12. Summary of the physical properties, major ions, nutrients, and trace elements in Devonian aquifer near Cedar Rapids, Linn County, lowa, $1993-97$.

[Data are summarized from the U.S. Geological Survey (USGS) National Water Information System database (USGS, 2020); search using the parameter code to see full parameter names, descriptions, and other data in the database. See well locations on figure 5. $n$, number of samples; Min, minimum; Med, median, Max, maximum; mg/L, milligram per liter; $\mu \mathrm{S} / \mathrm{cm}$ at $25^{\circ} \mathrm{C}$, microsiemens per centimeter at 25 degrees Celsius; ${ }^{\circ} \mathrm{C}$, degree Celsius; $\mathrm{SiO}_{2}$, silicon dioxide; $\mathrm{NH}_{4}^{+}$, ammonium; $\mathrm{N}$, nitrogen; P, phosphorus; $\mu \mathrm{g} / \mathrm{L}$, microgram per liter]

\begin{tabular}{|c|c|c|c|c|c|c|c|c|c|c|c|c|c|c|}
\hline \multirow[t]{2}{*}{ Constituent } & \multirow{2}{*}{$\begin{array}{c}\text { USGS } \\
\text { parameter } \\
\text { code }\end{array}$} & \multirow{2}{*}{$\begin{array}{c}\text { Unit of } \\
\text { measurement }\end{array}$} & \multicolumn{4}{|c|}{$\begin{array}{l}\text { Seminole well field, monitoring well } \\
\text { CRM6 }\end{array}$} & \multicolumn{4}{|c|}{ West well field, monitoring well CRM7 } & \multicolumn{4}{|c|}{ East well field, monitoring well CRM12 } \\
\hline & & & $n$ & Min & Med & Max & $n$ & Min & Med & Max & $n$ & Min & Med & Max \\
\hline \multicolumn{15}{|c|}{ Physical properties } \\
\hline $\begin{array}{r}\text { Dissolved } \\
\text { oxygen }\end{array}$ & 00300 & $\mathrm{mg} / \mathrm{L}$ & 9 & 0.1 & 0.1 & 0.4 & 10 & 0.1 & 0.3 & 1.8 & 6 & 0.1 & 0.1 & 2 \\
\hline $\mathrm{pH}$ & 00400 & Standard units & 9 & 7.1 & 7.4 & 8.4 & 10 & 7.2 & 7.4 & 7.7 & 6 & 7.0 & 7.4 & 8.1 \\
\hline $\begin{array}{l}\text { Specific } \\
\text { conductance }\end{array}$ & 00095 & $\mu \mathrm{S} / \mathrm{cm}$ at $25^{\circ} \mathrm{C}$ & 9 & 375 & 443 & 580 & 10 & 500 & 585 & 632 & 6 & 588 & 646 & 790 \\
\hline $\begin{array}{l}\text { Temperature, } \\
\text { water }\end{array}$ & 00010 & ${ }^{\circ} \mathrm{C}$ & 9 & 9.0 & 10.3 & 11.9 & 11 & 6.8 & 10.6 & 13 & 6 & 8.2 & 9.5 & 11.0 \\
\hline \multicolumn{15}{|c|}{ Major ions } \\
\hline $\begin{array}{l}\text { Total dissolved } \\
\text { solids }\end{array}$ & 70300 & $\mathrm{mg} / \mathrm{L}$ & 7 & 190 & 242 & 254 & 8 & 290 & 328 & 345 & 5 & 358 & 393 & 437 \\
\hline Calcium & 00915 & $\mathrm{mg} / \mathrm{L}$ & 7 & 55.0 & 57.0 & 60.0 & 8 & 61.0 & 72.0 & 77.0 & 5 & 77.3 & 78.0 & 98.0 \\
\hline Magnesium & 00925 & $\mathrm{mg} / \mathrm{L}$ & 7 & 22.0 & 23.0 & 23.0 & 8 & 22.0 & 26.0 & 27.0 & 5 & 24.3 & 26.8 & 34.0 \\
\hline Potassium & 00935 & $\mathrm{mg} / \mathrm{L}$ & 7 & 1.20 & 1.30 & 1.90 & 8 & 1.70 & 3.05 & 5.00 & 5 & 2.88 & 3.30 & 3.80 \\
\hline Sodium & 00930 & $\mathrm{mg} / \mathrm{L}$ & 7 & 3.90 & 4.30 & 5.20 & 8 & 9.30 & 9.50 & 11.0 & 5 & 12.4 & 16.0 & 16.0 \\
\hline Bicarbonate & 00453 & $\mathrm{mg} / \mathrm{L}$ & 3 & 237 & 289 & 304 & 4 & 327 & 339 & 354 & 3 & 370 & 378 & 389 \\
\hline Carbonate & 00452 & $\mathrm{mg} / \mathrm{L}$ & 3 & $<0.1$ & $<0.1$ & $<0.1$ & 4 & $<0.1$ & $<0.1$ & $<0.1$ & 3 & $<0.1$ & $<0.1$ & $<0.1$ \\
\hline Chloride & 00940 & $\mathrm{mg} / \mathrm{L}$ & 7 & 0.50 & 0.60 & 3.5 & 8 & 14.0 & 19.0 & 21.0 & 5 & 24.8 & 29.0 & 34.2 \\
\hline Sulfate & 00945 & $\mathrm{mg} / \mathrm{L}$ & 7 & 6.3 & 7.2 & 11.0 & 8 & 14.0 & 21.0 & 31.0 & 5 & 11.2 & 15.0 & 22.0 \\
\hline Fluoride & 00950 & $\mathrm{mg} / \mathrm{L}$ & 7 & 0.2 & 0.3 & 0.4 & 8 & 0.2 & 0.3 & 0.3 & 5 & 0.1 & 0.2 & 0.2 \\
\hline Silica & 00955 & $\mathrm{mg} / \mathrm{L}$ as $\mathrm{SiO}_{2}$ & 7 & 13.0 & 13.0 & 14.0 & 8 & 9.0 & 14.0 & 15.0 & 5 & 17.4 & 18.0 & 20.0 \\
\hline \multicolumn{15}{|c|}{ Nutrients } \\
\hline $\begin{array}{c}\text { Ammonia }\left(\mathrm{NH}_{3}\right. \\
\left.\text { plus } \mathrm{NH}_{4}^{+}\right)\end{array}$ & 00608 & $\mathrm{mg} / \mathrm{L}$ as $\mathrm{N}$ & 5 & 0.26 & 0.28 & 0.33 & 6 & 0.19 & 1.7 & 3.8 & 4 & 3.0 & 3.4 & 3.7 \\
\hline $\begin{array}{l}\text { Nitrate plus } \\
\text { nitrite }\end{array}$ & 00631 & $\mathrm{mg} / \mathrm{L}$ as $\mathrm{N}$ & 5 & 0.05 & 0.05 & 0.11 & 6 & 0.05 & 0.05 & 0.29 & 4 & 0.05 & 0.05 & 0.06 \\
\hline Nitrate & 00618 & $\mathrm{mg} / \mathrm{L}$ as $\mathrm{N}$ & 5 & 0.05 & 0.05 & 0.11 & 6 & 0.05 & 0.05 & 0.28 & 4 & 0.05 & 0.05 & 0.057 \\
\hline Nitrite & 00613 & $\mathrm{mg} / \mathrm{L}$ as $\mathrm{N}$ & 5 & 0.01 & 0.01 & 0.01 & 6 & 0.01 & 0.01 & 0.01 & 4 & 0.01 & 0.01 & 0.01 \\
\hline Orthophosphate & 00671 & $\mathrm{mg} / \mathrm{L}$ as $\mathrm{P}$ & 5 & 0.01 & 0.01 & 0.01 & 6 & 0.02 & 0.03 & 0.05 & 4 & 0.01 & 0.01 & 0.01 \\
\hline
\end{tabular}


[Data are summarized from the U.S. Geological Survey (USGS) National Water Information System database (USGS, 2020); search using the parameter code to see full parameter names, descriptions, and other data in the database. See well locations on figure 5. n, number of samples; Min, minimum; Med, median, Max, maximum; mg/L, milligram per liter; $\mu \mathrm{S} / \mathrm{cm}$ at $25^{\circ} \mathrm{C}$, microsiemens per centimeter at 25 degrees Celsius; ${ }^{\circ} \mathrm{C}$, degree Celsius; $\mathrm{SiO}_{2}$, silicon dioxide; $\mathrm{NH}_{4}{ }^{+}$, ammonium; $\mathrm{N}$, nitrogen; $\mathrm{P}$, phosphorus; $\mu \mathrm{g} / \mathrm{L}$, microgram per liter]

\begin{tabular}{|c|c|c|c|c|c|c|c|c|c|c|c|c|c|c|}
\hline \multirow{2}{*}{ Constituent } & \multirow{2}{*}{$\begin{array}{l}\text { USGS } \\
\text { parameter } \\
\text { code }\end{array}$} & \multirow{2}{*}{$\begin{array}{c}\text { Unit of } \\
\text { measurement }\end{array}$} & \multicolumn{4}{|c|}{$\begin{array}{l}\text { Seminole well field, monitoring well } \\
\text { CRM6 }\end{array}$} & \multicolumn{4}{|c|}{ West well field, monitoring well CRM7 } & \multicolumn{4}{|c|}{ East well field, monitoring well CRM12 } \\
\hline & & & $n$ & Min & Med & Max & $n$ & Min & Med & Max & $n$ & Min & Med & Max \\
\hline \multicolumn{15}{|c|}{ Nutrients-Continued } \\
\hline $\begin{array}{c}\text { Dissolved } \\
\text { organic } \\
\text { carbon }\end{array}$ & 00681 & $\mathrm{mg} / \mathrm{L}$ & 7 & 0.20 & 0.40 & 0.60 & 7 & 0.40 & 1.8 & 2.9 & 5 & 1.7 & 1.9 & 2.5 \\
\hline \multicolumn{15}{|c|}{ Trace elements } \\
\hline Bromide & 71870 & $\mathrm{mg} / \mathrm{L}$ & 7 & 0.02 & 0.02 & 0.04 & 8 & 0.01 & 0.04 & 0.05 & 5 & 0.03 & 0.05 & 0.1 \\
\hline Iron & 01046 & $\mu \mathrm{g} / \mathrm{L}$ & 7 & 3.0 & 5.0 & 16 & 8 & 3.0 & 140 & 920 & 5 & 3,200 & 3,500 & 3,900 \\
\hline Manganese & 01056 & $\mu \mathrm{g} / \mathrm{L}$ & 7 & 3.0 & 4.0 & 7.0 & 8 & 120 & 1,140 & 2,200 & 5 & 550 & 616 & 720 \\
\hline Boron & 01020 & $\mu \mathrm{g} / \mathrm{L}$ & 6 & 40 & 48 & 50 & 5 & 40 & 40 & 50 & 5 & 44 & 48 & 60 \\
\hline
\end{tabular}


Table 13. Summary of the physical properties, major ions, nutrients, and trace elements in the Cedar River near Cedar Rapids, Linn County, lowa, $1990-2019$.

[Data are summarized from the U.S. Geological Survey (USGS) National Water Information System database (USGS, 2020); search using the parameter code to see full parameter names, descriptions, and other data in the database. $n$, number of samples; $\mathrm{mg} / \mathrm{L}$, milligram per liter; $\mu \mathrm{S} / \mathrm{cm}$ at $25{ }^{\circ} \mathrm{C}$, microsiemens per centimeter at 25 degrees Celsius; ${ }^{\circ} \mathrm{C}$, degree $\mathrm{Celsius;}<$, less than; $\mathrm{SiO}_{2}$, silicon dioxide; $\mathrm{NH}_{4}{ }^{+}$, ammonium; N, nitrogen; P, phosphorus; E, estimated; $\mu \mathrm{g} / \mathrm{L}$, microgram per liter]

\begin{tabular}{|c|c|c|c|c|c|c|c|c|c|}
\hline \multirow[b]{2}{*}{ Constituent } & \multirow{2}{*}{$\begin{array}{l}\text { USGS parameter } \\
\text { code }\end{array}$} & \multirow{2}{*}{$\begin{array}{c}\text { Unit of measure- } \\
\text { ment }\end{array}$} & \multirow[b]{2}{*}{$n$} & \multicolumn{6}{|c|}{ Summary statistics } \\
\hline & & & & Minimum & $\begin{array}{c}\text { 25th } \\
\text { percentile }\end{array}$ & Median & Mean & $\begin{array}{c}\text { 75th } \\
\text { percentile }\end{array}$ & Maximum \\
\hline \multicolumn{10}{|c|}{ Physical properties } \\
\hline Dissolved oxygen & 00300 & $\mathrm{mg} / \mathrm{L}$ & 142 & 3.1 & 8.8 & 11.5 & 11.2 & 13.1 & 18.4 \\
\hline $\mathrm{pH}$ & 00400 & Standard units & 143 & 6.0 & 7.8 & 8.2 & 8.2 & 8.4 & 9.7 \\
\hline Specific conductance & 00095 & $\mu \mathrm{S} / \mathrm{cm}$ at $25^{\circ} \mathrm{C}$ & 147 & 234 & 430 & 516 & 499 & 562 & 730 \\
\hline Temperature, water & 00010 & ${ }^{\circ} \mathrm{C}$ & 143 & -0.1 & 5.0 & 15.4 & 13.5 & 21.0 & 27.1 \\
\hline \multicolumn{10}{|c|}{ Major ions } \\
\hline Total dissolved solids & 70300 & $\mathrm{mg} / \mathrm{L}$ & 110 & 154 & 261 & 303 & 299 & 342 & 470 \\
\hline Calcium & 00915 & $\mathrm{mg} / \mathrm{L}$ & 117 & 23.2 & 50.7 & 66.3 & 62.6 & 75 & 97.7 \\
\hline Magnesium & 00925 & $\mathrm{mg} / \mathrm{L}$ & 117 & 8.1 & 18.6 & 20.9 & 20.1 & 22.7 & 28.5 \\
\hline Potassium & 00935 & $\mathrm{mg} / \mathrm{L}$ & 111 & 1.35 & 2.08 & 2.45 & 2.67 & 2.95 & 7.20 \\
\hline Sodium & 00930 & $\mathrm{mg} / \mathrm{L}$ & 117 & 2.50 & 7.60 & 9.74 & 10.5 & 12.8 & 24.6 \\
\hline Bicarbonate & 00453 & $\mathrm{mg} / \mathrm{L}$ & 111 & $<0.1$ & 165 & 212 & 206 & 249 & 316 \\
\hline Carbonate & 00452 & $\mathrm{mg} / \mathrm{L}$ & 103 & $<0.1$ & 0.3 & 1.3 & 5.8 & 2.7 & 203 \\
\hline Chloride & 00940 & $\mathrm{mg} / \mathrm{L}$ & 120 & 5.79 & 18.0 & 21.9 & 22.4 & 26.1 & 42.2 \\
\hline Sulfate & 00945 & $\mathrm{mg} / \mathrm{L}$ & 117 & 0.12 & 23.7 & 29.1 & 29.0 & 35.7 & 47.1 \\
\hline Fluoride & 00950 & $\mathrm{mg} / \mathrm{L}$ & 117 & $<0.10$ & 0.20 & 0.21 & 0.22 & 0.24 & 0.31 \\
\hline Silica & 00955 & $\mathrm{mg} / \mathrm{L}$ as $\mathrm{SiO}_{2}$ & 117 & 0.02 & 5.07 & 9.66 & 8.64 & 12.0 & 20.3 \\
\hline \multicolumn{10}{|c|}{ Nutrients } \\
\hline Ammonia $\left(\mathrm{NH}_{3}\right.$ plus $\left.\mathrm{NH}_{4}^{+}\right)$ & 00608 & $\mathrm{mg} / \mathrm{L}$ as $\mathrm{N}$ & 139 & $<0.04$ & $<0.04$ & $<0.04$ & 0.08 & $<0.04$ & 1.5 \\
\hline Nitrate plus nitrite & 00631 & $\mathrm{mg} / \mathrm{L}$ as $\mathrm{N}$ & 147 & $<0.04$ & 3.48 & 5.60 & 5.65 & 7.30 & 12.6 \\
\hline Nitrate & 00618 & $\mathrm{mg} / \mathrm{L}$ as $\mathrm{N}$ & 141 & $<0.029$ & 3.47 & 5.65 & 5.64 & 7.23 & 12.5 \\
\hline Nitrite & 00613 & $\mathrm{mg} / \mathrm{L}$ as $\mathrm{N}$ & 141 & 0.002 & 0.011 & 0.017 & 0.024 & 0.028 & 0.128 \\
\hline Orthophosphate & 00671 & $\mathrm{mg} / \mathrm{L}$ as $\mathrm{P}$ & 147 & E0.003 & 0.01 & 0.07 & 0.10 & 0.14 & 0.60 \\
\hline Dissolved organic carbon & 00681 & $\mathrm{mg} / \mathrm{L}$ & 100 & 1.50 & 2.60 & 3.01 & 3.83 & 3.86 & 41.0 \\
\hline \multicolumn{10}{|c|}{ Trace elements } \\
\hline Bromide & 71870 & $\mathrm{mg} / \mathrm{L}$ & 96 & $<0.01$ & 0.02 & 0.03 & 0.03 & 0.03 & 0.2 \\
\hline Iron & 01046 & $\mu \mathrm{g} / \mathrm{L}$ & 115 & E2.1 & 7.00 & 10.0 & 32.0 & 18.1 & 1,300 \\
\hline Manganese & 01056 & $\mu \mathrm{g} / \mathrm{L}$ & 114 & 1.48 & 3.66 & 6.43 & 13.3 & 10.0 & 384 \\
\hline Boron & 01020 & $\mu \mathrm{g} / \mathrm{L}$ & 97 & 10 & 21 & 27 & 27 & 32 & 49 \\
\hline
\end{tabular}


the detection rate of these historically heavily used compounds (Baker and Stone, 2015) to $<20$ percent. The maximum alachlor concentration did not exceed the EPA (2018) MCL of $2.0 \mu \mathrm{g} / \mathrm{L}$. Although not monitored throughout the entire study period, Boyd (2000) found that additional degradation compounds of atrazine, alachlor, and metolachlor were detected more frequently and at greater concentrations than their corresponding parent herbicides.

Temporal changes in the water quality in the Devonian aquifer and the Cedar River that are the source of a large part of the water in the alluvial aquifer can cause similar changes in the alluvial aquifer near Cedar Rapids, Iowa. In contrast to water in the Devonian aquifer, water quality in the Cedar River was highly variable and changed temporally through the study period. A previous study described an increase in nitrate $-\mathrm{N}$ concentration from $<1.0 \mathrm{mg} / \mathrm{L}$ in the early $1900 \mathrm{~s}$ to $>5.0 \mathrm{mg} / \mathrm{L}$ in the 2000s in the Cedar River at Cedar Rapids (Kalkhoff, 2018). Using daily estimated nitrate-N concentrations, a small but statistically significant $(p<0.05)$ increase in the annual nitrate- $\mathrm{N}$ concentration (fig. 14) was observed in the Cedar River. Although nitrate-N concentrations were variable from year to year, estimated annual concentrations increased from about $5.1 \mathrm{mg} / \mathrm{L}$ in 1990 to $6.6 \mathrm{mg} / \mathrm{L}$ in 2020 . Nitrate-N concentrations increased at a rate of $0.05 \mathrm{mg} / \mathrm{L}$ per year (fig. 14). Annual mean nitrate-N concentrations ranged from $3.67 \mathrm{mg} / \mathrm{L}$ during an extremely dry year (2012) to $8.95 \mathrm{mg} / \mathrm{L}$ during a wet year (2016).

Annual means are important to understanding changes in the basin, but as a source of water to the alluvial aquifer, day to day concentrations in the Cedar River would allow the municipality to ensure their supply to customers meets the drinking-water standard (MCL) of $10.0 \mathrm{mg} / \mathrm{L}$. Changes in the number of days annually that the water in the Cedar River exceeds the drinking-water standard provides important information that can be used by the City to manage the water supply and provides a measure of the progress in nutrient reduction efforts upstream in the Cedar River Basin.

There was no significant ( $p<0.05$, Kendal Tau) trend in the number of days annually the nitrate- $\mathrm{N}$ concentration was $10 \mathrm{mg} / \mathrm{L}$ or more in water flowing into Cedar Rapids, Iowa (fig. 17) during the study period. However, nitrate-N concentrations tended to exceed $10 \mathrm{mg} / \mathrm{L}$ during more days annually during the last decade (2010-19) than in the previous two decades (1990-2009) of the study. In 2016, more than 100 days exceeded the MCL, though the number of exceedances were much lower in 2017-19. There were no measured MCL exceedances in 2019 even though the annual streamflow in the Cedar River was the second greatest on record (fig. 2). The reason for the wide range in annual nitrate- $\mathrm{N}$ exceedances is beyond the scope of this report, but climate and land use may be important factors.

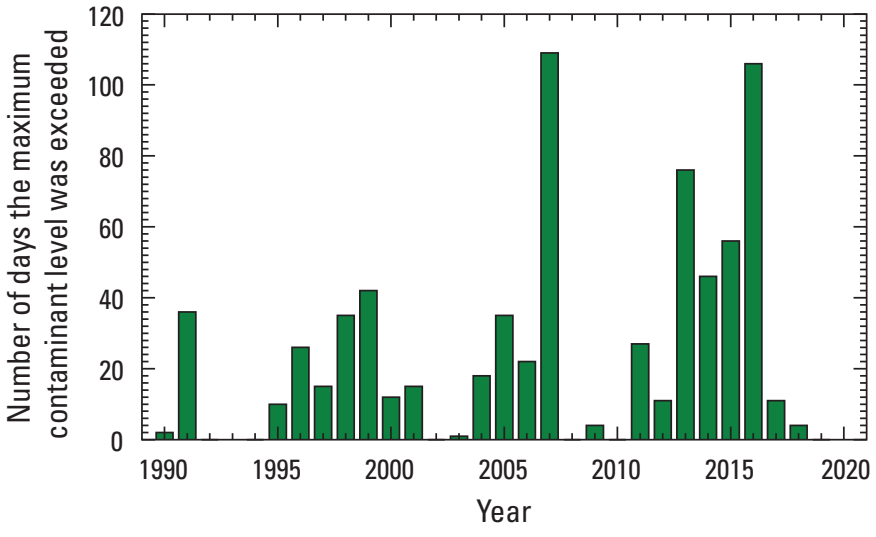

[Data from Meppelink and Kalkhoff (2021); the maximum contaminant level is 10 milligrams per liter (U.S. Environmental Protection Agency, 2018)]

Figure 17. Number of days the maximum contaminant level for nitrate plus nitrite nitrogen in drinking water was exceeded in the Cedar River near Palo (surface-water site SW-1), Linn County, lowa, 1990-2019. 


\section{Relation Between Water Quality of the Alluvial Aquifer and the Devonian Aquifer}

Water from the underlying Devonian aquifer can dilute shallow groundwater pumped from the alluvial aquifer because of the lower concentrations of major ions and nutrients and the general absence of synthetic organic compounds. Devonian aquifer water can enhance the concentration of several reduced compounds in water pumped from the alluvial aquifer.

Although overall dissolved-solids concentrations in the Devonian aquifer are in the same range as those from the alluvial aquifer, the median concentration $(283 \mathrm{mg} / \mathrm{L})$ in samples from the Devonian aquifer (table 11) was smaller than the median in samples from the three well types in the alluvial aquifer (312-318 mg/L, fig. 9). Nitrate-N was not frequently detected (32 percent of samples) with a median concentration less than the detection level in Devonian aquifer samples. In contrast, nitrate- $\mathrm{N}$ was detected in 84 percent of the alluvial aquifer samples with a median concentration of about $3.0 \mathrm{mg} / \mathrm{L}$ in alluvial aquifer samples. Chloride and sulfate, two constituents that may occur naturally and may originate from anthropogenic sources, were present in significantly $(p<0.05)$ smaller concentrations in the Devonian aquifer (table 11) than in the alluvial aquifer (table 6).

Reducing conditions (limited dissolved oxygen) generally found in the Devonian aquifer allow for increased ammonia, iron, and manganese. Although about 50 percent of the samples were greater than the SMCL of $50 \mu \mathrm{g} / \mathrm{L}$, manganese concentrations in the Devonian aquifer were commonly less than those in the alluvial aquifer (fig. 9). In contrast, iron concentrations were significantly $(p<0.05$, Wilcoxon Rank Sum test) greater in the Devonian aquifer than in the alluvial aquifer (fig. 9).

Synthetic organic compounds were rarely detected and if detected were present in small concentrations (table 7) in the Devonian aquifer. Only atrazine, the atrazine degradate (CIAT), metolachlor, prometon, and metribuzin were detected. These compounds were detected in 20 percent or less of the samples at concentrations $<0.10 \mu \mathrm{g} / \mathrm{L}$ (table 7). The Devonian aquifer would not contribute substantial amounts of atrazine or other commonly used pesticides to water pumped from the alluvial aquifer.

\section{Relation Between Water Quality of the Alluvial Aquifer and the Cedar River}

The pumping of large-capacity production wells induces flow from the Cedar River into the alluvial aquifer. The induced flow transports dissolved constituents through the streambed and streambanks into the aquifer. In addition, as groundwater flows through the aquifer from the river, reactions can occur that alter the chemical composition of the water (Boyd, 1999; Schnoebelen, 2008). Many of these reactions are microbially catalyzed redox reactions (Schulmeyer and Schnoebelen, 1998; Boyd, 1999) that result in the depletion of dissolved oxygen and DOC, removal of nitrate-N, and the mobilization of trace elements such as iron and manganese.

\section{Dissolved Oxygen}

Natural and induced flow of oxygenated water from the Cedar River can transport oxygen to the riparian zone of the alluvial aquifer. Dissolved-oxygen concentrations abruptly decrease in the first 50-65 ft of streambank sediments (aquifer). The observed decrease of about 2 to $4 \mathrm{mg} / \mathrm{L}$ in the dissolved-oxygen concentration between the Cedar River and vertical production well Sem10 (fig. 18) may be the result of mineralization of dissolved organic compounds by microorganisms (Bourg and Bertin, 1993) and mixing with anoxic water from other parts of the aquifer. Estimated travel times from Cedar River to vertical production well Sem10 ranged from 7 to 17 days. River water with high oxygen concentrations that infiltrates into the groundwater had slightly more than 2 weeks or less residence time in the aquifer before it was pumped out for consumption (Schulmeyer, 1995). Under pumping conditions, there was insufficient time for biological uptake to eliminate dissolved oxygen. However, when pumping ceases, groundwater levels return to levels similar to the river and anoxic conditions are soon reestablished in the aquifer (fig. 18).

\section{Nitrogen}

Nitrate-N concentrations in the Cedar River can exceed the MCL of $10 \mathrm{mg} / \mathrm{L}$ for drinking water (fig. 9; Meppelink and others, 2019), but nitrate- $\mathrm{N}$ concentrations in the alluvial wells are generally lower. Median nitrate-N concentration of samples from the vertical production and Ranney production wells were 1.44 to $2.91 \mathrm{mg} / \mathrm{L}$ less than the median concentration in samples from the Cedar River (fig. 9). However, nitrate- $\mathrm{N}$ attenuation of induced flow from the Cedar River can be much greater in individual wells and at different times of the year. For example, concentrations in one well, Ranney production well Ran3 (fig. 19), slightly lagged the temporal pattern of the Cedar River, but the daily concentration was about $4.05 \mathrm{mg} / \mathrm{L}$ smaller in the well than in the river during March 12 to December 21, 2015. Daily nitrate-N concentrations were frequently greater than the $10.0 \mathrm{mg} / \mathrm{L} \mathrm{MCL}$ in the Cedar River but did not exceed the MCL in Ran3. Nitrate-N concentrations in the Cedar River respond rapidly to changes in streamflow, but the response was muted in the alluvial aquifer (fig. 19). The nitrate-N attenuation was not spatially uniform in the study area. The attenuation of daily nitrate- $\mathrm{N}$ concentrations from the Cedar River to Ranney production 


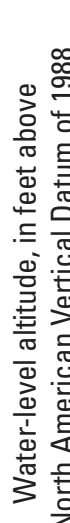

号
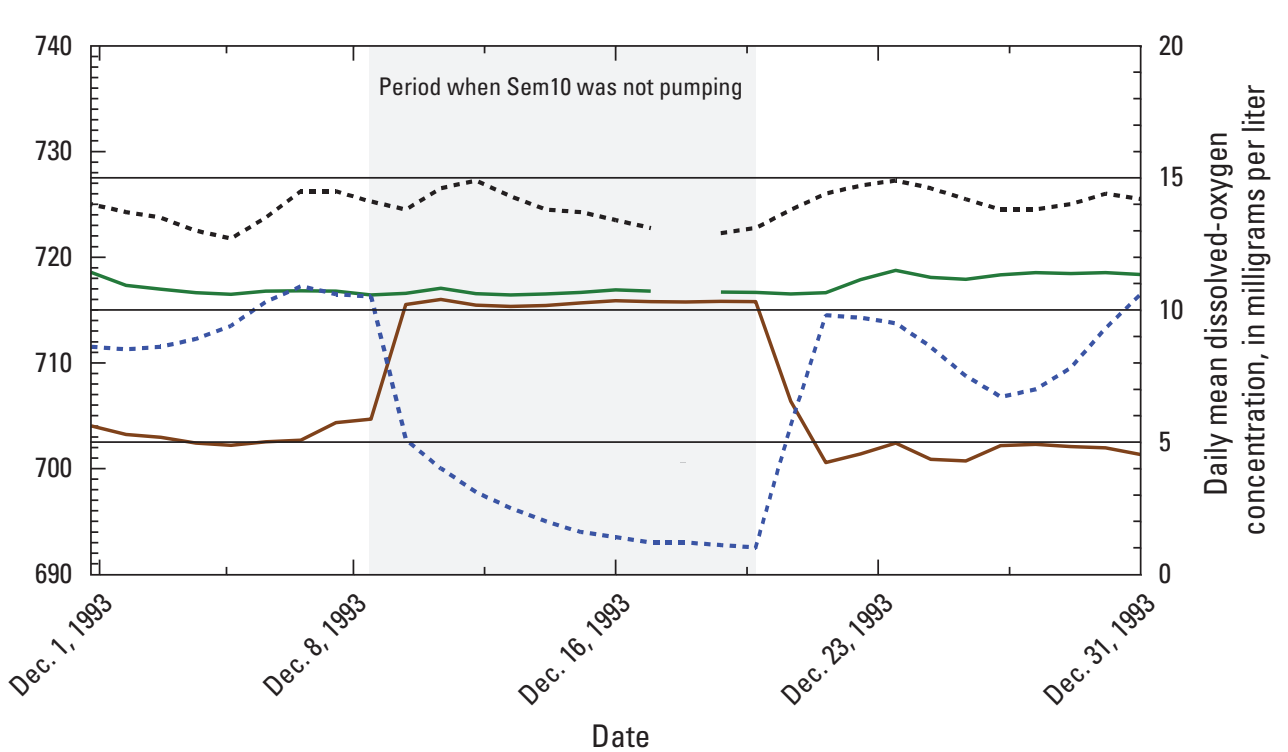

EXPLANATION

[Data from Schnoebelen and Schulmeyer (1996)]

Altitude

_ $\quad$ Surface water site SW-2
_ Vertical production well Sem10
$\quad$ Dissolved oxygen
.... $\quad$ Surface water site SW-2
... $\quad$ Vertical production well Sem10

Figure 18. Water-level altitude and dissolved oxygen concentration in the Cedar River (surface-water site SW-2) and vertical production well Sem10, Linn County, lowa, December 1993.

well Ran6 had a mean of $2.83 \mathrm{mg} / \mathrm{L}$ during March 12 to December 21, 2015, which was about 70 percent of the mean attenuation measured in the alluvial aquifer at Ran3. Well construction, geology, and geochemical properties may account for spatial differences in nitrate- $\mathrm{N}$ attenuation in the alluvial aquifer in the Cedar Rapids well fields.

Nitrate reduction probably occurred throughout the well fields in Cedar Rapids because raw water from multiple wells (before any treatment) generally had smaller nitrate- $\mathrm{N}$ concentrations than in the river (Littin, 2012; Meppelink and others, 2019). Several factors that may cause this nitrate reduction are denitrification in the aquifer or dilution by water from other parts of the alluvial aquifer or the deeper bedrock aquifer with smaller concentrations.

In contrast to nitrate- $\mathrm{N}$, ammonia-N concentrations in samples from the Cedar River were significantly $(p<0.05$, Kruskal-Wallis test) less than in samples from the alluvial aquifer (fig. 9). Ammonia-N in the alluvial aquifer is mainly produced by the mineralization of organic matter and inflow of small amounts from the Cedar River. Ammonia-N is minimally present in the Cedar River (fig. 9, table 13) because ammonia entering the aerated river environment is rapidly converted to nitrate by nitrifying bacteria. Ammonia-N concentrations in samples from the Cedar River normally $<0.04 \mathrm{mg} / \mathrm{L}$ (table 13), whereas ammonia- $\mathrm{N}$ concentrations in samples from the alluvial aquifer were larger, normally ranging from 0.02 to $0.14 \mathrm{mg} / \mathrm{L}$ (table 6).

\section{Iron and Manganese}

The chemical composition of water infiltrating from the Cedar River into the shallow zone of the alluvial aquifer (about 10 to $23 \mathrm{ft}$ below land surface) can be substantially altered by redox reactions (Boyd, 1999). Normally, anoxic conditions are prevalent in the alluvial aquifer next to the Cedar River, but with the induced infiltration of oxygenated river water, redox conditions may be altered such that naturally occurring dissolved iron and manganese precipitate out of solution.

Infiltration of river water may also transport additional organic matter to the aquifer that would contribute to the biological reduction of oxygen and subsequent denitrification of nitrate- $\mathrm{N}$ in the aquifer. McMahon and others (2019) found that manganese and DOC concentrations were higher in shallow groundwater near rivers than in areas distant from rivers indicating that DOC infiltrating the alluvial aquifer supports 


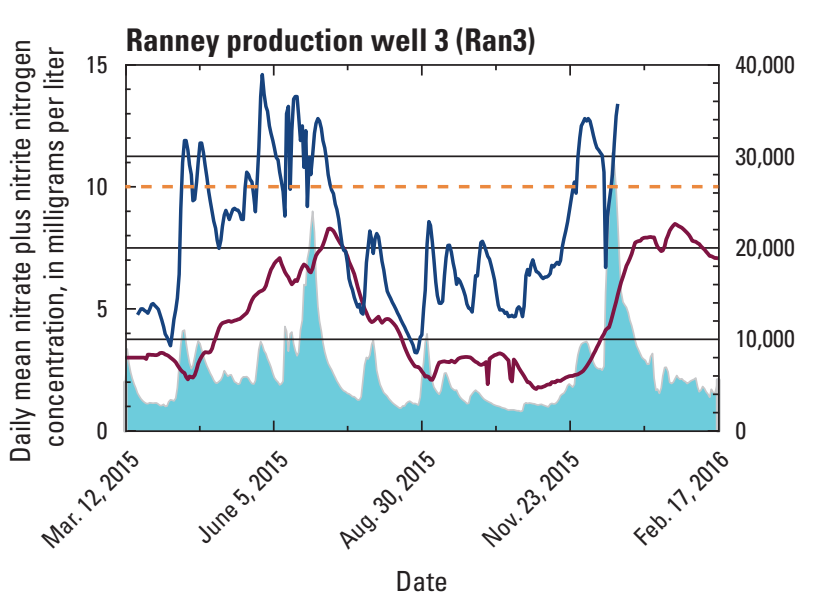

EXPLANATION
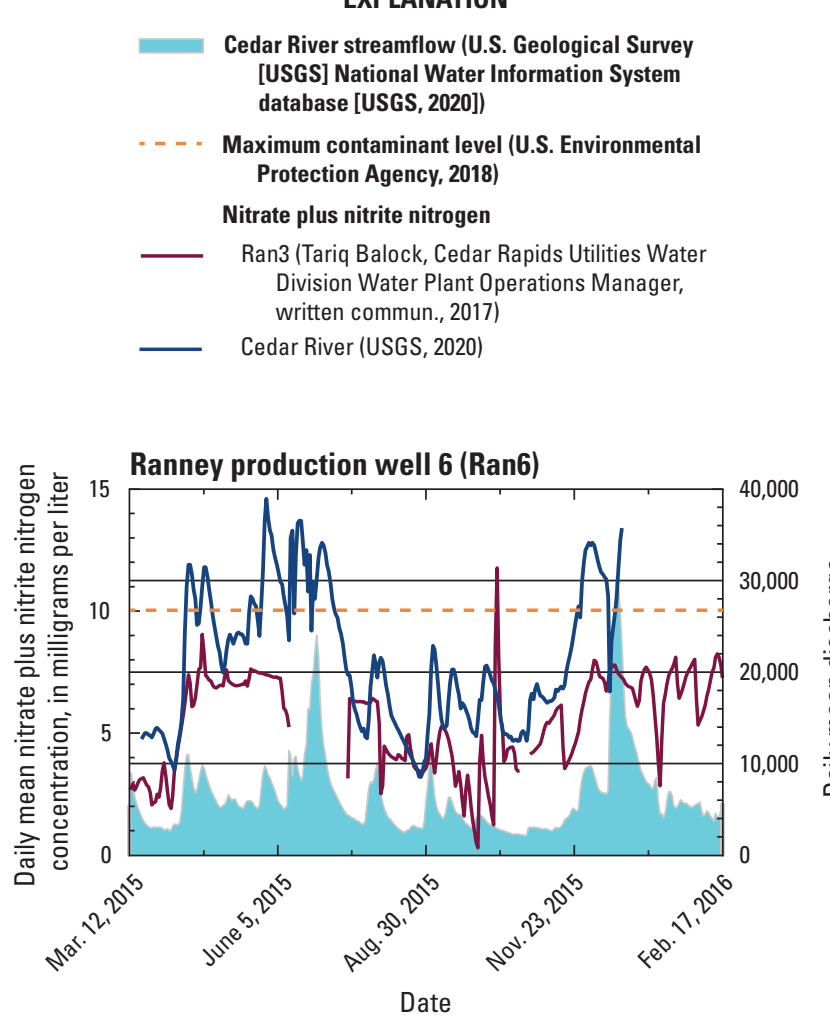

EXPLANATION

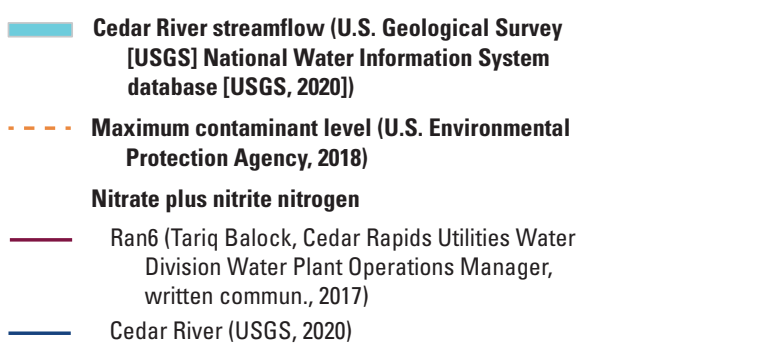

Figure 19. Relation between nitrate plus nitrite nitrogen concentration in the alluvial aquifer (Ranney production wells Ran3 and Ran6) and the Cedar River (surface-water site SW-1), Linn County, lowa, 2015-16. manganese dissolution. The median DOC concentration in the Cedar River was significantly $(p<0.05)$ greater than in the alluvial aquifer and thus could contribute DOC to the aquifer.

Concentrations of iron and manganese in the alluvial aquifer near the Cedar River are also affected by pumping of large-capacity production wells. For example, manganese concentrations are highly variable over time in monitoring well CRM22 that was between the Cedar River and production well Sem4. Manganese concentrations from CRM22 ranged from less than the detection level of $10 \mu \mathrm{g} / \mathrm{L}$ to $2,320 \mu \mathrm{g} / \mathrm{L}$. Manganese concentrations were inversely related to nitrate- $\mathrm{N}$ concentrations. When manganese was present in samples from CRM22, nitrate-N concentrations generally were small or less than the detection level (fig. 13). Nitrate-N concentrations generally $>1.0 \mathrm{mg} / \mathrm{L}$ were associated with oxic conditions (dissolved-oxygen concentrations $>1.0 \mathrm{mg} / \mathrm{L}$ ) in the alluvial aquifer (fig. 13). Although pumping data for well Seminole 4 are not available, the oxygenated water with the presence of nitrate- $\mathrm{N}$ indicates that pumping can induce flow from the Cedar River into the alluvial aquifer. Conversely, when pumping ceases, microbes quickly consume the dissolved oxygen and reduce nitrate creating anoxic conditions in the aquifer. Boyd (1999) found that dissolved oxygen in the alluvial aquifer was depleted within about $10 \mathrm{ft}$ from the banks of the Cedar River. Low oxygen or anoxic conditions are conducive to the dissolution of iron and manganese from aquifer material.

\section{Pesticides}

The concentration gradient of pesticides from the Cedar River to the alluvial aquifer and the limited application of pesticide products in the Cedar Rapids well fields indicate that pesticides are transported from the river to the alluvial aquifer. Under conditions when the aquifer was not stressed by pumping, atrazine concentrations increased within $100 \mathrm{ft}$ of the river when concentrations in the Cedar River increased (Squillace, and others, 1996; Liszewski and Squillace, 1991). Annual mean concentrations in the Cedar River for several of the commonly applied herbicides in the Cedar River Basin are substantially greater than in the alluvial aquifer (table 7). Differences varied yearly, but annual median concentrations of atrazine, acetochlor, and metolachlor were several micrograms per liter greater in the Cedar River than in the alluvial aquifer. The lower concentrations in the aquifer probably were caused by degradation of the pesticide in the aquifer or, like other water quality constituents, diluted with water from recharge areas. Pesticides were not detected in recharge areas of the aquifer (Boyd, 2000) and were present infrequently and at small concentrations in the underlying Devonian aquifer (table 7). Herbicide occurrence in groundwater was related to their persistence in aerobic environments (Barbash and others, 2001), but the occurrence may not be uniform spatially through the aquifer because of different geochemical environments. Microcosm experiments have shown 
(Luo and others, 2019) that some pesticide compounds degrade in anerobic environments in the presence of dissolved organic matter. Atrazine, acetochlor, and metolachlor degradate compounds were observed in relatively high concentrations in shallow monitoring wells near the Cedar River in the Seminole well field in the 1990s (Boyd, 2000).

\section{Flooding Effect on Alluvial Water Quality}

Floodwater that inundates the floodplain next to the Cedar River was an occasional source of water and potential contaminants to the Cedar River alluvial aquifer. Nutrients and pesticides transported in the Cedar River are present in floodwaters that may seep through the soil into the aquifer. Inundation of the floodplain during flood conditions can affect nutrient concentrations in alluvial aquifers (Kolpin and Thurman, 1995). Seepage from floodwaters was not quantified during this study, but continuous monitoring and discrete water quality data provide a qualitive view of floodwater's potential effect on the alluvial water quality.

A previous study (Hubbard and others, 2011) found that nitrate- $\mathrm{N}$ concentrations in rivers during major floods were generally less than during more typical flows. Two example floods on the Cedar River in the study area show the concentration of nitrate-N in floodwater was seasonally variable and more dilute than in the river before and after the flood (fig. 20).

A series of rain events in June and July 2014 inundated the alluvial flood plain in the study area. Nitrate-N concentrations during the first flood inundation period in June 2014 were initially high $(11.7 \mathrm{mg} / \mathrm{L})$ but decreased slightly to a minimum concentration of $9.91 \mathrm{mg} / \mathrm{L}$ near peak flow. Nitrate-N concentrations increased to preflood levels when the Cedar River returned to its banks on June 27, 2014. The nitrate-N concentration in the floodwaters during the 5-day inundation period was $11.0 \mathrm{mg} / \mathrm{L}$, at least $1.0 \mathrm{mg} / \mathrm{L}$ lower than concentrations before and after the flood. In contrast to the small decrease in nitrate- $\mathrm{N}$ concentration during June flood conditions, nitrate- $\mathrm{N}$ concentrations decreased substantially during the flood in July. Nitrate-N concentrations decreased from $11.7 \mathrm{mg} / \mathrm{L}$ before the flood to $3.16 \mathrm{mg} / \mathrm{L}$ soon after the Cedar River inundated the floodplain (fig. 20). The mean nitrate- $\mathrm{N}$ concentration in floodwater during the 6-day July inundation period was $7.63 \mathrm{mg} / \mathrm{L}$, about $4.0 \mathrm{mg} / \mathrm{L}$ less than concentrations before and after inundation.

Another example of the dilution of nitrate concentrations during floodplain inundation was a series of late summer and early fall floods in 2018 . These floods were not as great as the early summer 2014 floods, but concentrations were also diluted during the peak flow when floodwaters inundated the floodplain (fig. 20). Floodwaters inundated the floodplain for 8 days in September and 6 days in October. The mean nitrate- $\mathrm{N}$ concentration of the inundation water was $4.24 \mathrm{mg} / \mathrm{L}$ in September and $4.42 \mathrm{mg} / \mathrm{L}$ in October. The mean nitrate- $\mathrm{N}$ concentration of the inundation water was about $0.75 \mathrm{mg} / \mathrm{L}$ lower than the concentration before and after the flood in the Cedar River.
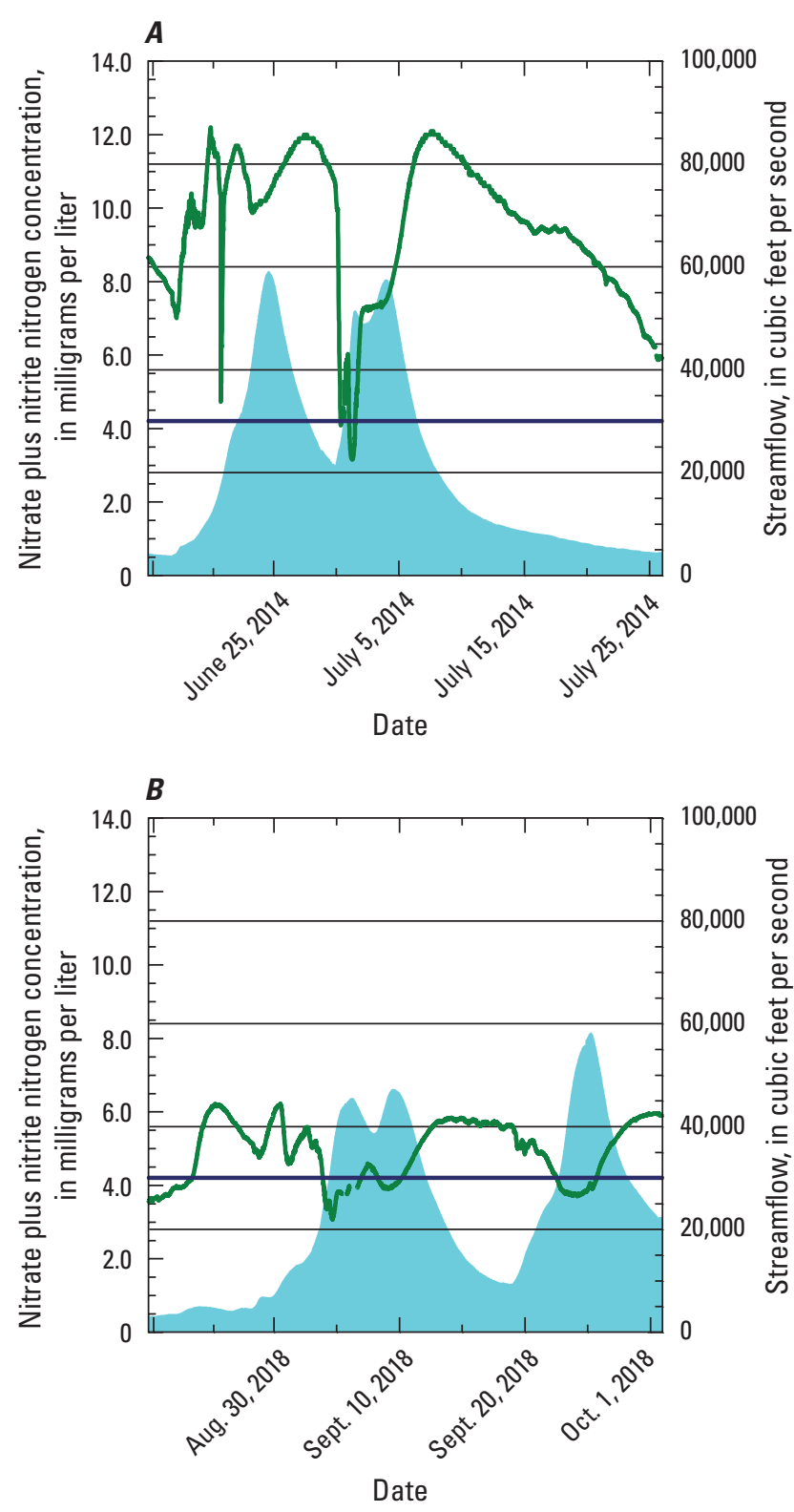

EXPLANATION

[Data from the U.S. Geological Survey (USGS) National Water Information System database (USGS, 2020); streamflow and 2014 concentrations from Cedar River at surface-water site SW-4; 2018 concentrations from Cedar River at surface-water site SW-1]

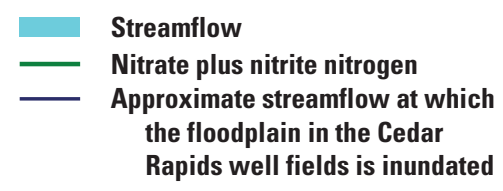

Figure 20. Nitrate plus nitrite nitrogen concentration in floodwaters of the Cedar River, Linn County, lowa. $A$, June and July 2014. B, August and September 2018. 
Additionally, floodplain inundation water was generally more dilute than pre- and post-floodwater in the Cedar River with respect to major ions and trace elements (table 14). The median dissolved-solids concentration in the flood samples was substantially less than in the remainder of the samples. This pattern was reflected in smaller median concentrations of all the major ions, except silica, when the floodplain was inundated (table 14). The median bromide, manganese, and boron concentrations in flood samples were also less than the median concentration in the nonfloodwater samples.
Exceptions to the smaller constituent concentrations in flood samples were constituents that are commonly transported in overland runoff. These constituents include orthophosphate, silica, and iron, which may be flushed from the land surface during heavy rains and transported with sediment and organic carbon as part of the organic matter component of runoff (table 14). The median orthophosphate concentration for flood samples from the Cedar River during this study $(0.018 \mathrm{mg} / \mathrm{L})$ was similar to the median of samples from 19 river sites in eastern and central Iowa collected during major flooding in 2008 (Hubbard and others, 2011).

Table 14. Median values of physical properties, major ions, nutrients, and trace elements in the Cedar River during normal and flood conditions, Linn County, lowa, 1990-2019.

[Data are summarized from the U.S. Geological Survey (USGS) National Water Information System database (USGS, 2020); search using the parameter code to see full parameter names, descriptions, and other data in the database. $n$, number of samples; $\mathrm{mg} / \mathrm{L}$, milligram per liter; $\mu \mathrm{S} / \mathrm{cm}$, microsiemens per centimeter at 25 degrees Celsius; ${ }^{\circ} \mathrm{C}$, degree Celsius; $\mathrm{SiO}_{2}$, silicon dioxide; $\mathrm{NH}_{4}{ }^{+}$, ammonium; $\mathrm{N}$, nitrogen; $\mathrm{P}$, phosphorus; $\mu \mathrm{g} / \mathrm{L}$ microgram per liter]

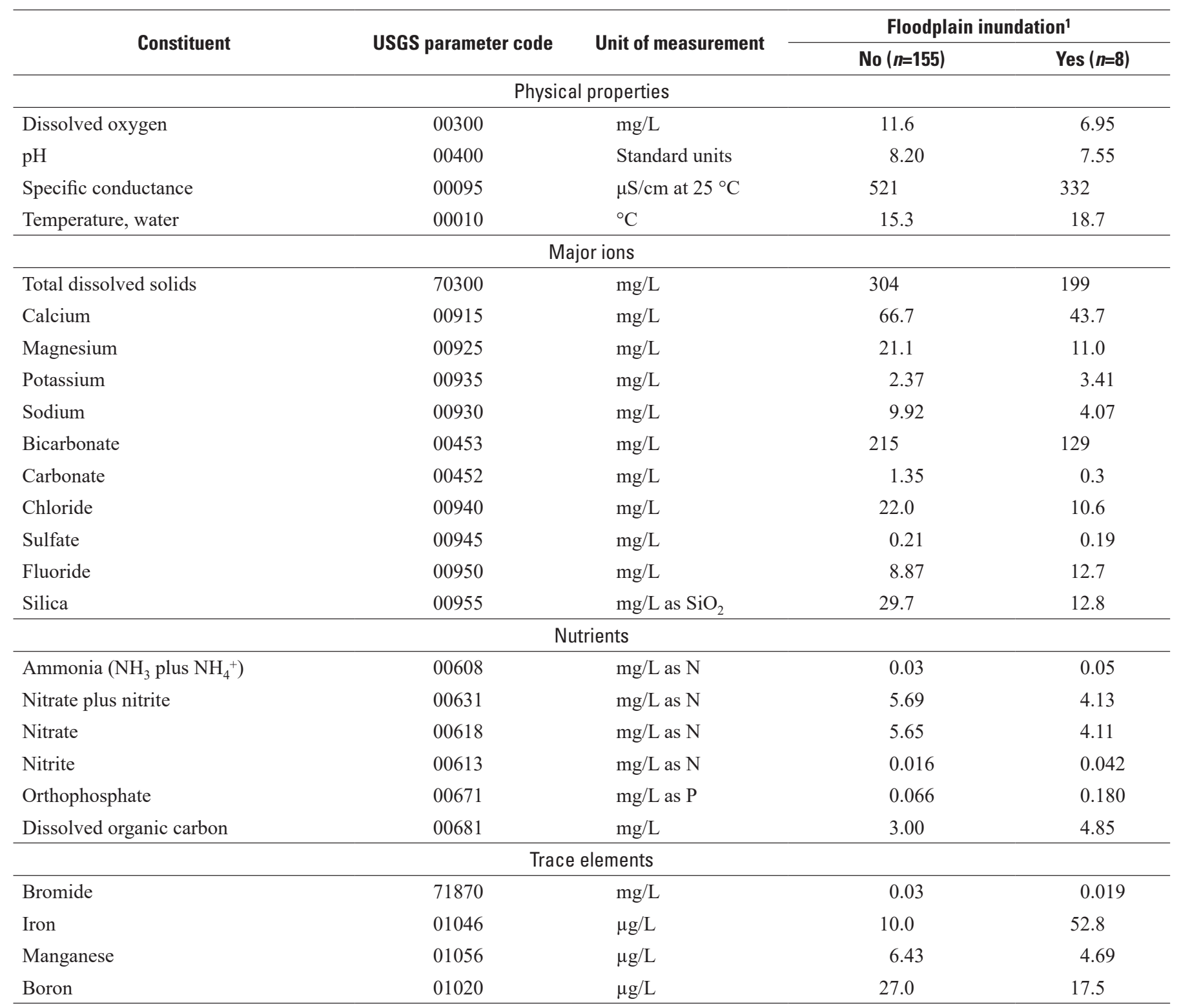

${ }^{1}$ Floodplain inundation occurs when streamflow in the Cedar River at Cedar Rapids, Iowa, is greater than or equal to 30,000 cubic feet per second. 


\section{Summary and Conclusion}

Sand and gravel deposits along rivers and streams (surficial alluvial aquifers) are an important source of water supply for residential and industrial purposes in Iowa. Alluvial aquifers in Iowa have a greater percentage of wells with nitrate plus nitrite nitrogen (nitrate-N) exceeding drinking-water standards than other aquifers; are susceptible to contamination by organic contaminants; and have high concentrations of naturally occurring iron and manganese in depositional areas that contain abundant organic matter. The Cedar River alluvial aquifer in Linn County, Iowa, is used extensively as a source of water for residential and industrial purposes. The aquifer was studied from 1990 to 2019 to understand the effect of large-capacity production well pumping on spatial and temporal hydrologic and water-quality variability. Withdrawal of large quantities of water for residential and industrial purposes has altered the normal flow of water in the alluvial aquifer. Pumping induces flow from the Cedar River and the underlying Devonian aquifer into the alluvial aquifer.

The purpose of this report was to summarize and document hydrologic and water-quality data collected from 1990 through 2019 in the Cedar River alluvial aquifer, from the underlying bedrock aquifer (Devonian aquifer), and the Cedar River, waters that contribute water to the production wells. The summary included analysis of temporal and spatial trends and description of how water quality in the aquifer was affected by differences in pumping, geochemical conditions, and changes in river water quality.

The alluvial aquifer is part of a complex hydrologic system where water moves between several unconsolidated and bedrock formations and the Cedar River. The alluvial aquifer is directly recharged by infiltration of rainfall and snowmelt and water moving laterally from the adjacent upland till and loess deposits and is discharged in the Cedar River. Withdrawal of large quantities of water for residential and industrial purposes induces flow into the alluvial aquifer from the Cedar River and from the underlying Devonian aquifer.

The water in the alluvial aquifer was generally a calcium bicarbonate type that was present in an environment of a narrow range of $\mathrm{pH}$ (7.1 to 7.4 standard units) with low dissolvedoxygen concentrations ( 75 percent of samples less than or equal to 2.1 milligrams per liter $[\mathrm{mg} / \mathrm{L}])$. Concentrations of major ions in the Cedar River alluvial aquifer were relatively uniform spatially and temporally during the study period. Dissolved nitrate plus nitrite nitrogen (nitrate- $\mathrm{N}$ ) concentrations normally ranged from 0.52 to $5.14 \mathrm{mg} / \mathrm{L}$; a few samples ( 2 percent) exceeded the maximum contaminant level of $10 \mathrm{mg} / \mathrm{L}$ for drinking water. Concentrations of iron and manganese exceeded secondary drinking-water standards in 11.0 and 66.8 percent, respectively, of the samples from the alluvial aquifer. Triazine and acetoanolide preemergent herbicides were the most commonly detected pesticide compounds. The triazine compounds, atrazine and one of its primary degradates, 2-chloro-4-isopropylamino-6-amino$s$-triazine (CIAT), were present in more than 90 percent of all samples from the alluvial aquifer. Two acetoanalide herbicides, metolachlor and acetochlor, were detected in 87.7 and
27.5 percent of the samples, respectively. Only one other compound, the triazene herbicide prometon used for nonrow crop agricultural and urban weed control, was detected in more than 20 percent of the samples. A total of 32 pesticide compounds were detected in 1 or more samples during the study.

Concentrations of reduction-oxidation-affected constituents such as nitrate- $\mathrm{N}$, ammonia as nitrogen, iron, and manganese varies in groundwater from the Seminole well field to the East well field, following the upstream to downstream gradient of the overlying Cedar River. Nitrate- $\mathrm{N}$ concentrations decreased; and ammonia as nitrogen, iron, and manganese increased downstream from the Seminole well field to the East well field. High nitrate- $\mathrm{N}$ concentrations were associated with a freeflowing reach of the Cedar River. High ammonia as nitrogen, iron, and manganese concentrations in the alluvial aquifer were associated with a regulated reach of the Cedar River. Water in the alluvial aquifer near backwater wetlands had nitrate-N concentrations that were four to six times lower when compared to those in the Cedar River but had iron and manganese concentrations an order of magnitude higher when compared to the river. Wetlands and regulated reaches of the river have fine-grained bottom sediment that impede flow into the alluvial aquifer allowing sufficient time for bacteria using organic matter to deplete the dissolved oxygen. In these anoxic conditions, nitrate- $\mathrm{N}$ was reduced to elemental nitrogen by bacterially mediated denitrification and are favorable for the dissolution of minerals containing oxidized forms of iron and manganese.

The major ion concentrations in the alluvial aquifer, while variable, did not change through the study. The annual median dissolved solids concentration varied within a small range from 288 to $345 \mathrm{mg} / \mathrm{L}$ and was normally between 309 and $326 \mathrm{mg} / \mathrm{L}$. Nitrate-N concentrations increased during the study. Annual mean concentrations of atrazine and acetochlor decreased in the Cedar River alluvial aquifer during the study period. Annual mean acetochlor concentrations remained relatively constant in the range from 0.04 to 0.06 microgram per liter until 2015 when mean annual concentrations decreased to 0.03 microgram per liter or less. The degradation compound, 2-chloro-6-ethylamino4-amino-s-triazine (CEAT), followed the annual concentration trend of its parent compound, atrazine, during the study period.

Pumping by large-capacity production wells caused induced flow from the underlying Devonian aquifer and adjacent Cedar River altering the water chemistry in the alluvial aquifer. Water in the Devonian aquifer can dilute water in the alluvial aquifer in relation to dissolved solids, nitrate-N, and pesticides but can enhance the concentration of iron and manganese, two reduced compounds, in water pumped from the alluvial aquifer. Induced flow from the Cedar River transports dissolved nitrate-N and pesticide compounds through the streambed and streambanks into the alluvial aquifer. In addition, as water flows through the aquifer from the river, microbially catalyzed reduction-oxidation reactions occur that deplete dissolved oxygen and dissolved organic carbon, remove nitrate-N, and mobilize trace elements such as iron and manganese. 
During the 1990-2019 study period, streamflow in the Cedar River was great enough to flow into the backwater channels and wetlands during about 7.5 percent of the days and completely inundated the alluvial floodplain on about 1 percent of the days. Floodwater, containing excess nutrients and pesticides, inundating the floodplain next to the Cedar River was a potential source of water and potential contaminants to the Cedar River alluvial aquifer.

This report documents hydrologic and water-quality conditions in the Cedar River alluvial aquifer during the 1990-2019 study period and provides information that can assist the City of Cedar Rapids Utilities Water Department in managing groundwater resources to provide needed quantities of water to their residential and industrial users. This report provides information that can be used for development of additional water supplies as the anticipated demand for water increases as city of Cedar Rapids continues to expand and as land use in and near the city and upstream in the Cedar River Basin continue to change. Hydrologic and water-quality data documented in this report can form the basis on which current and future coordinated nutrient reduction practices upstream in the Cedar River Basin can be evaluated.

\section{References Cited}

American Public Health Association, American Water Works Association, and Water Environment Federation, 2017, 5310 total organic carbon (TOC), in Part 5000, Aggregate organic constituents, of Standard methods for the examination of water and wastewater: American Public Health Association, American Water Works Association, and Water Environment Federation, 9 p., accessed October 2018 at https://doi.org/10.2105/SMWW.2882.104.

Baker, N.T., and Stone, W.W., 2015, Estimated annual agricultural pesticide use for counties of the conterminous United States, 2008-12: U.S. Geological Survey Data Series 907, 9 p., accessed April 2020 at https://doi.org/10.3133/ds907.

Barbash, J.E., Thelin, G.P., Kolpin, D.W., and Gilliom, R.J., 2001, Major herbicides in ground water-Results from the National Water-Quality Assessment: Journal of Environmental Quality, v. 30, no. 3, p. 831-845, accessed August 2020 at https://doi.org/10.2134/jeq2001.303831x.

Bourg, A.C.M., and Bertin, C., 1993, Biogeochemical processes during the infiltration of river water into an alluvial aquifer: Environmental Science \& Technology, v. 27, no. 4, p. 661-666, https://pubs.acs.org/doi/pdf/10.1021/ es00041a009, accessed August 2021 at https://doi.org/ 10.1021/es00041a009.
Boyd, R.A., 1998, Characterizing ground water flow in the municipal well fields of Cedar Rapids, Iowa, with selected environmental tracers: Journal of the American Water Resources Association, v. 34, no. 3, p. 507-518, accessed May 2018 at https://doi.org/10.1111/j.17521688.1998.tb00950.x.

Boyd, R.A., 1999, Groundwater geochemistry in the Seminole well field, Cedar Rapids, Iowa: Journal of the American Water Resources Association, v. 35, no. 5, p. 1257-1268, accessed May 2018 at https://doi.org/10.1111/j.17521688.1999.tb04212.x.

Boyd, R.A., 2000, Herbicides and herbicide degradates in shallow groundwater and the Cedar River near a municipal well field, Cedar Rapids, Iowa: The Science of the Total Environment, v. 248, no. 2-3, p. 241-253, accessed May 2018 at https://doi.org/10.1016/S0048-9697(99)00546-X.

Boyd, R.A., Kuzniar, R.L., and Schulmeyer, P.M., 1999, Selected hydrologic data from the Cedar Rapids area, Linn County, Iowa, April 1996 through March 1999: U.S. Geological Survey Open-File Report 99-461, 241 p., accessed May 2018 at https://doi.org/10.3133/ofr99461.

Buchmiller, R.C., 1995, Hydrogeologic and agriculturalchemical data for the South Skunk River alluvial aquifer at a site in Story County, Iowa, 1992-93: U.S. Geological Survey Water-Resources Investigations Report 94-4244, 29 p., accessed August 2019 at https://doi.org/10.3133/ wri944244.

Burkart, M.E., Simpkins, W.W., Squillace, P.J., and Helmke, M., 1999, Tributary stream infiltration as a source of herbicides in an alluvial aquifer: Journal of Environmental Quality, v. 28, no. 1, p. 69-74, accessed August 2019 at https://doi.org/10.2134/jeq1999.00472425002800010007x.

Cedar Rapids, 2020, Middle Cedar Partnership Project: City of Cedar Rapids, Iowa, website, accessed November 16, 2020, at http://www.cedar-rapids.org/residents/utilities/middle cedar_partnership_project.php.

Clarke, M.L., and Thamke, J.N., 1988, Estimated water use in Iowa, 1985: U.S. Geological Survey Open-File Report 87-704, 28 p., accessed May 2019 at https://doi.org/ 10.3133/ofr87704.

Cunningham, W.L., and Schalk, C.W., comps., 2011, Groundwater technical procedures of the U.S. Geological Survey: U.S. Geological Survey Techniques and Methods, book 1, chap. A1, 151 p., accessed April 2021 at https://doi.org/10.3133/tm1A1. 
Fishman, M.J., ed., 1993, Methods of analysis by the U.S. Geological Survey National Water Quality LaboratoryDetermination of inorganic and organic constituents in water and fluvial sediments: U.S. Geological Survey Open-File Report 93-125, 217 p. accessed June 2021 at https://doi.org/10.3133/ofr93125.

Foreman, W.T., Majewski, M.S., Goolsby, D.A., Wiebe, F.E., and Coupe, R.H., 2000, Pesticides in the atmosphere of the Mississippi River Valley, part II-Air: The Science of the Total Environment, v. 248, no. 2-3, p. 213-226, accessed October 2019 at https://doi.org/10.1016/S00489697(99)00544-6.

Haj, A.E., Ha, W.S., Gruhn, L.R., Bristow, E.L., Gahala, A.M., Valder, J.F., Johnson, C.D., White, E.A., and Sterner, S.P., 2021, Conceptual and numerical groundwater flow model of the Cedar River alluvial aquifer system with simulation of drought stress on groundwater availability near Cedar Rapids, Iowa, for 2011 through 2013: U.S. Geological Survey Scientific Investigations Report 2021-5065, 59 p., accessed September 2021 at https://doi.org/10.3133/ $\operatorname{sir} 20215065$.

Hansen, R.E., 1970, Geology and ground-water resources of Linn County, Iowa: Iowa Geological Survey WaterSupply Bulletin 10, 67 p., accessed November 2018 at https://www.iihr.uiowa.edu/igs/publications/uploads/ WSB-10.pdf.

Helsel, D.R., Hirsch, R.M., Ryberg, K.R., Archfield, S.A., and Gilroy, E.J., 2020, Statistical methods in water resources: U.S. Geological Survey Techniques and Methods, book 4, chap. A3, 458 p., accessed May 2020 at https://doi.org/ $10.3133 / \mathrm{tm} 4 \mathrm{~A} 3$.

Hubbard, L., Kolpin, D.W., Kalkhoff, S.J., and Robertson, D.M., 2011, Nutrient and sediment concentrations and corresponding loads during the historic June 2008 flooding in eastern Iowa: Journal of Environmental Quality, v. 40, no. 1, p. 166-175, accessed August 2020 at https://doi.org/ 10.2134/jeq2010.0257.

Iowa Department of Natural Resources, 2006, Total maximum daily load for nitrate, Cedar River, Linn County, Iowa: Iowa Department of Natural Resources Watershed Improvement Section, 55 p., accessed April 2018 at https://www.iowadnr.gov/Portals/idnr/uploads/water/ watershed/tmdl/files/final/cedarriver.pdf.

Iowa Department of Natural Resources, 2020a, Alluvial deposits in Iowa: Iowa Geospatial Data, accessed June 2021 at https://geodata.iowa.gov/documents/iowadnr:alluvialdeposits-in-iowa/about.

Iowa Department of Natural Resources, 2020b, AQuIA water quality monitoring: Iowa Department of Natural Resources database, accessed May 2020 at https://programs.iowadnr.gov/aquia/Sites/10570002.
Iowa Department of Natural Resources, 2020c, Watershed management authorities in Iowa: Iowa Department of Natural Resources web page, accessed November 2020 at https://www.iowadnr.gov/Environmental-Protection/WaterQuality/Watershed-Management-Authorities.

Iowa Flood Information System [IFIS], 2019, Iowa flood information system inundation maps Cedar Rapids: University of Iowa, Iowa Flood Center website, accessed August 2019 at https://ifis.iowafloodcenter.org/ifis/.

Iowa State Planning Board, 1938, Mineral analysis of the underground waters of Iowa. Iowa State Planning Board, $123 \mathrm{p}$.

Iowa State University, 2014, Iowa nutrient reduction strategy-A science and technology-based framework to assess and reduce nutrients to Iowa waters and the Gulf of Mexico: Iowa Department of Agriculture and Land Stewardship, Iowa Department of Natural Resources, and Iowa State University College of Agriculture and Life Sciences, accessed November 16, 2020, at http://www.nutrientstrategy.iastate.edu/sites/default/files/ documents/NRSfull-141001.pdf.

Iowa Watershed Approach, 2020, Home: Iowa Watershed Approach website, accessed November 16, 2020, at https://iowawatershedapproach.org.

Johnson, C.D., Bristow, E.L., White, E.A., Gruhn, L.R., Pappas, K.L., Phillips, S.N., and Lane, J.W., Jr., 2020, Geophysical data collected in the Cedar River floodplain, Cedar Rapids, Iowa, 2015-2017: U.S. Geological Survey data release, accessed April 2021 at https://doi.org/10.5066/ P9YXJDHX.

Kalkhoff, S.J., 2018, Transport of nitrogen and phosphorus in the Cedar River Basin, Iowa and Minnesota, 2000-15: U.S. Geological Survey Scientific Investigations Report 2018-5090, 44 p., accessed April 2021 at https://doi.org/ 10.3133/sir20185090.

Kolpin, D.W., and Thurman, E.M., 1995, Postflood occurrence of selected agricultural chemicals and volatile organic compounds in near-surface unconsolidated aquifers in the upper Mississippi River Basin, 1993: U.S. Geological Survey Circular 1120-G, 20 p., accessed September 2019 at https://doi.org/10.3133/cir1120G.

Liszewski, M.J., and Squillace, P.J., 1991, The effect of surface-water and ground-water exchange on the transport and storage of atrazine in the Cedar River, Iowa, in Mallard, G.E., and Aronson, D.A., eds., U.S. Geological Survey Toxic Substances Hydrology Program-Proceedings of the technical meeting, Monterey, Calif., March 11-15, 1991: U.S. Geological Survey Water-Resources Investigations Report 91-4034, p. 195-202, accessed September 2020 at https://doi.org/10.3133/wri914034. 
Littin, G.R., 2012, Selected water-quality data from the Cedar River and Cedar Rapids well fields, Cedar Rapids, Iowa, 2006-10: U.S. Geological Survey Data Series 657, 32 p., accessed May 2018 at https://doi.org/10.3133/ds657.

Littin, G.R., and Schnoebelen, D.J., 2010, Selected waterquality data from the Cedar River and Cedar Rapids well fields, Cedar Rapids, Iowa, 1999-2005: U.S. Geological Survey, Data Series 494, 52 p., accessed May 2018 at https://doi.org/10.3133/ds494.

Lorenz, D.L., 2015, smwrBase-An R package for managing hydrologic data, version 1.1.1: U.S. Geological Survey Open-File Report 2015-1202, 7 p., accessed April 2018 at https://doi.org/10.3133/ofr20151202.

Luo, Y., Atashgahi, S., Rijnaarts, H.H.M., Comans, R.N.J., and Sutton, N.B., 2019, Influence of different redox conditions and dissolved organic matter on pesticide biodegradation in simulated groundwater systems: The Science of the Total Environment, v. 677, p. 692-699, accessed May 2020 at https://doi.org/10.1016/j.scitotenv.2019.04.128.

Majewski, M.S., Foreman, W.T., and Goolsby, D.A., 2000, Pesticides in the atmosphere of the Mississippi River Valley, part I-Rain: The Science of the Total Environment, v. 248, no. 2-3, p. 201-212, accessed October 2019 at https://doi.org/10.1016/S0048-9697(99)00543-4.

McMahon, P.B., Belitz, K., Reddy, J.E., and Johnson, T.D., 2019, Elevated manganese concentrations in United States groundwater, role of land surface-soil-aquifer connections: Environmental Science \& Technology, v. 53, no. 1, p. 29-38, accessed February 2019 at https://doi.org/ 10.1021 /acs.est.8b04055.

Meppelink, S.M., and Kalkhoff, S.J., 2021, Hydrologic and water quality data from the Cedar River and Cedar River alluvial aquifer, Linn County, Iowa, 1990-2019: U.S. Geological Survey data release, https://doi.org/10.5066/ P9Z7VKOU.

Meppelink, S.M., Stelzer, E.A., Bristow, E.L., and Littin, G.R., 2019, Selected water-quality data from the Cedar River and Cedar Rapids well fields, Cedar Rapids, Iowa, 2008-17: U.S. Geological Survey Data Series 1110, 49 p., accessed April 2021 at https://doi.org/10.3133/ds1110.

National Oceanic and Atmospheric Administration [NOAA], 2019, Climate data online-Dataset discovery: National Oceanic and Atmospheric Administration digital data, accessed September 2019 at https://www.ncdc.noaa.gov/ cdo-web/datasets.

Norton, W.H., Hendrixson, W.S., Simpson, H.E., Meinzer, O.E., and others, 1912, Underground water resources of Iowa: U.S. Geological Survey Water Supply Paper 293, 994 p., accessed May 2018 at https://doi.org/10.3133/wsp293.
Patton, C.J., and Kryskalla, J.R., 2003, Methods of analysis by the U.S. Geological Survey National Water Quality Laboratory-Evaluation of alkaline persulfate digestion as an alternative to Kjeldahl digestion for determination of total and dissolved nitrogen and phosphorus in water: U.S. Geological Survey Water-Resources Investigations Report 2003-4174, 33 p., accessed April 2021 at https://doi.org/ $10.3133 /$ wri034174.

Patton, C.J., and Kryskalla, J.R., 2011, Colorimetric determination of nitrate plus nitrite in water by enzymatic reduction, automated discrete analyzer methods: U.S. Geological Survey Techniques and Methods, book 5, chap. B8, 34 p., accessed April 2021 at https://doi.org/10.3133/tm5B8.

Peck, A.M., and Hornbuckle, K.C., 2005, Gas-phase concentrations of current-use pesticides in Iowa: Environmental Science \& Technology, v. 39, no. 9, p. 2952-2959, accessed October 2019 at https://doi.org/10.1021/es0486418.

Pellerin, B.A., Bergamaschi, B.A., Downing, B.D., Saraceno, J.F., Garrett, J.A., and Olsen, L.D., 2013, Optical techniques for the determination of nitrate in environmental waters: Guidelines for instrument selection, operation, deployment, maintenance, quality assurance, and data reporting: U.S. Geological Survey Techniques and Methods, book 1, chap. D5, 37 p., accessed April 2021 at https://doi.org/ 10.3133/tm1D5.

Quade, D.J., Bettis, E.A., III, Ludvigson, G.A., Giglierano, J.P., and Slaughter, M.K., 1998, Surficial geologic materials of Linn County, Iowa: Iowa Department of Natural Resources, Geological Survey Bureau Open File Map Series 98-2, scale 1:100,000, accessed March 2020 at https:/www.iihr.uiowa.edu/igs/publications/uploads/ofm1998-2.pdf.

Rantz, S.E., 1982, Measurement and computation of streamflow-Volume 1. Measurement of stage and discharge: U.S. Geological Survey Water Supply Paper 2175, 284 p., accessed April 2021 at https://doi.org/ $10.3133 /$ wsp2 175 .

Sandstrom, M.W., Stroppel, M.E., Foreman, W.T., and Schroeder, M.P., 2001, Methods of analysis by the U.S. Geological Survey National Water Quality LaboratoryDetermination of moderate-use pesticides and selected degradates in water by $\mathrm{C}-18$ solid-phase extraction and gas chromatography/mass spectrometry: U.S. Geological Survey Water-Resources Investigations Report 01-4098, 70 p., accessed April 2021 at https://doi.org/10.3133/ wri20014098. 
Sandstrom, M.W., Wydoski, D.S., Schroeder, M.P., Zamboni, J.L., and Foreman, W.T., 1992, Methods of analysis by the U.S. Geological Survey National Water Quality Laboratory - Determination of organonitrogen herbicides in water by solid-phase extraction and capillary-column gas chromatography/mass spectrometry with selected-ion monitoring: U.S. Geological Survey Open-File Report 91-519, 26 p. accessed April 2021 at https://doi.org/10.3133/ ofr91519.

Sauer, V.B., and Turnipseed, D.P., 2010, Stage measurement at gaging stations: U.S. Geological Survey Techniques and Methods, book 3, chap. A7, 45 p., accessed April 2021 at https://doi.org/10.3133/tm3A7.

Savoca, M.E., Sadorf, E.M., Linhart, S.M., and Akers, K.K.B., 2000, Effects of land use and hydrogeology on the water quality of alluvial aquifers in eastern Iowa and Southern Minnesota, 1997: U.S. Geological Survey Water-Resources Investigations Report 99-4246, 38 p., accessed April 2021 at https://doi.org/10.3133/wri994246.

Schaap, B.D. and Linhart, S.M., 1998, Quality of ground water used for selected municipal water supplies in Iowa, 1982-96 water years: U.S. Geological Survey Open File Report 98-3, 67 p., accessed May 2019 at https://doi.org/ 10.3133/ofr983.

Schilling, K.E., 2002, Occurrence and distribution of ammonium in Iowa groundwater: Water Environment Federation, v. 74, no. 2, p. 177-186, accessed May 2010 at http://www. jstor.org/stable/25045590.

Schnoebelen, D.J., 2008, Effectiveness of an alluvial wetland on improving ground-water quality near a municipal well field, Cedar Rapids, Iowa, 1998-2006: U.S. Geological Survey Scientific Investigation Report 2008-5108, 29 p., accessed April 2021 at https://doi.org/10.3133/sir20085108.

Schnoebelen, D.J., and Schulmeyer, P.M., 1996, Selected hydrogeologic data from the Cedar Rapids area, Benton and Linn counties, Iowa, October 1992 through March 1996: U.S. Geological Survey Open File Report 96-471, 163 p., accessed April 2021 at https://doi.org/10.3133/ofr96471.

Schulmeyer, P.M., 1995, Effect of the Cedar River on the quality of the ground-water supply for Cedar Rapids, Iowa: U.S. Geological Survey Water-Resources Investigations Report 94-4211, 68 p., accessed April 2021 at https://doi.org/ 10.3133/wri944211.

Schulmeyer, P.M., Barnes, K.K. and Squillace, P.J., 1995, Hydrologic data from the lower Cedar River Basin, Iowa, 1989-91: U.S. Geological Survey Open-File Report 94-711-W, 116 p. accessed June 2021 at https://doi.org/ 10.3133/ofr94711W.
Schulmeyer, P.M., and Schnoebelen, D.J., 1998, Hydrogeology and water quality in the Cedar Rapids area, Iowa, 1992-96: U.S. Geological Survey Water-Resources Investigations Report 97-426, 77 p., accessed April 2021 at https://doi.org/10.3133/wri974261.

Siergieiev, D., Widerlund, A., Ingri, J., Lundberg, A., and Ohlander, B., 2014, Flow regulation effects on the hydrogeochemisty of the hyporheic zone in boreal rivers: The Science of the Total Environment, v. 499, p. 424-436, accessed April 2020 at https://doi.org/10.1016/j.scitotenv.2 014.06.112.

Squillace, P.J., 1996, Observed and simulated movement of bank-storage water: Ground Water, v. 34, no. 1, p. 121-134, accessed May 2018 at https://doi.org/10.1111/j.17456584.1996.tb01872.x.

Squillace, P.J., Caldwell, J.P., Schulmeyer, P.M., and Harvey, C.A., 1996, Movement of agricultural chemicals between surface water and ground water, lower Cedar River Basin, Iowa: U.S. Geological Survey Water Supply Paper 2448, 59 p., accessed May 2018 at https://doi.org/10.3133/wsp2448.

Squillace, P.J., and Engberg, R.A., 1988, Surface-water quality of the Cedar River Basin, Iowa-Minnesota, with emphasis on the occurrence and transport of herbicides, May 1984 through November 1985: U.S. Geological Survey WaterResources Investigations Report 88-4060, 81 p., accessed November 2015 at https://doi.org/10.3133/wri884060.

Struzeski, T.M., DeGiacomo, W.J., and Zayhowski, E.J., 1996, Methods of analysis by the U.S. Geological Survey National Water Quality Laboratory-Determination of dissolved aluminum and boron in water by inductively coupled plasmaatomic emission spectrometry: U.S. Geological Survey Open-File Report 96-149, 17 p., accessed April 2021 at https://doi.org/10.3133/ofr96149.

Stumm, W., and Morgan, J.J., 1981, Aquatic chemistryAn introduction emphasizing chemical equilbria in natural waters 2nd ed.: New York, Wiley-Interscience Publishers, $780 \mathrm{p}$.

Turco, M.J., and Buchmiller, R.C., 2004, Simulation of ground-water flow in the Cedar River alluvial aquifer flow system, Cedar Rapids, Iowa: U.S. Geological Survey Scientific Investigations Report 2004-5130, 39 p., accessed April 2021 at https://doi.org/10.3133/sir20045130.

U.S. Environmental Protection Agency, [EPA], 2018, 2018 edition of the drinking water standards and health advisories tables: Washington, D.C., U.S. Environmental Protection Agency Office of Water EPA 822-F-18-001, 12 p. accessed March 2020 at https://www.epa.gov/sites/production/files/ 2018-03/documents/dwtable2018.pdf. 
U.S. Fish and Wildlife Service, 2020, National Wetlands Inventory database: U.S. Fish and Wildlife Service database, accessed October 16, 2020, at https://www.fws.gov/ wetlands/.

U.S. Geological Survey, [USGS], 2019, Estimated annual agricultural pesticide use data: U.S. Geological Survey database and maps, accessed August 2020 at https://water.usgs.gov/ nawqa/pnsp/usage/maps/.

U.S. Geological Survey, [USGS], 2020, USGS water data for the Nation: U.S. Geological Survey National Water Information System (NWIS) database, accessed March 2020 at https://doi.org/10.5066/F7P55KJN. [Data for Iowa directly accessible at https://waterdata.usgs.gov/ia/ nwis/nwis.]

U.S. Geological Survey [USGS], variously dated, National field manual for the collection of water-quality data: U.S. Geological Survey Techniques of Water-Resources Investigations, book 9, chaps. A1-A10, accessed April 2018 at https://www.usgs.gov/mission-areas/water-resources/ science/national-field-manual-collection-water-qualitydata-nfm?qt-science_center_objects $=0 \#$ \#t-science center_objects.
Valder, J.F., Haj, A.E., Bristow, E.L., and Valseth, K.J., 2018, Delineation of selected lithologic units using airborne electromagnetic data near Cedar Rapids, Iowa: U.S. Geological Survey Scientific Investigations Map 3423, 2 sheets, 9-p. pamphlet, accessed April 2021 at https://doi.org/ 10.3133/sim3423.

Wahl, K., and Bunker, B.J., 1986, Hydrology of carbonate aquifers in southwestern Linn County and adjacent parts of Benton, Iowa, and Johnson Counties, Iowa: Iowa Geological Survey Water Supply Bulletin 15, 56 p. accessed April 2021 at https://www.iihr.uiowa.edu/igs/publications/ uploads/2014-10-23_09-10-46_wsb-15.pdf.

Wahl, K.D., Ludvigson, G.A., Ryan, G.L., and Steinkampf, W.C., 1978, Water resources of east-central Iowa: Iowa Geological Survey Water Atlas 6, accessed September 2019 at https://www.iihr.uiowa.edu/igs/publications/publications? category $=$ Water-Atlases.

Wang, W., and Squillace, P., 1994, Herbicide interchange between a stream and the adjacent alluvial aquifer: Environmental Science \& Technology, v. 28, no. 13, p. 2336-2344, accessed March 2020 at https://doi.org/ 10.1021/es00062a018.

Winter, T.C., Harvey, J.W., Franke, O.L., and Alley, W.M.,1998, Ground water and surface water-A single resource: U.S. Geological Survey Circular 1139, 79 p., accessed May 2020 at https://doi.org/10.3133/cir1139. 


\section{Appendix 1. Pesticide Compounds Not Detected in the Cedar River Alluvial and Devonian Aquifers and the Cedar River near Cedar Rapids, Linn County, lowa, 1990-2019}

Table 1.1. Pesticide compounds not detected in the Cedar River alluvial aquifer, Devonian aquifer, and the Cedar River near Cedar Rapids, Linn County, lowa, 1990-2019.

[Search in the U.S. Geological Survey (USGS) National Water Information System database (https://doi.org/ $10.5066 /$ F7P55KJN) using the parameter code to see full parameter names, descriptions, and other data in the database. EPTC, S-ethyl dipropylthiocarbamate]

\begin{tabular}{|c|c|}
\hline USGS parameter code & Pesticide compound \\
\hline 49295 & 1-Naphthol \\
\hline 82660 & 2,6-Diethylaniline \\
\hline 61618 & 2-Chloro-2,6-diethylacetanilide \\
\hline 61625 & 3,4-Dichloroaniline \\
\hline 61627 & 3,5-Dichloroaniline \\
\hline 61633 & 4-Chloro-2-methylphenol \\
\hline 34362 & $\alpha$-Endosulfan \\
\hline 82686 & Azinphos-methyl \\
\hline 61635 & Azinphos-methyl oxon \\
\hline 04029 & Bromacil \\
\hline 04028 & Butylate \\
\hline 82680 & Carbaryl \\
\hline 82674 & Carbofuran \\
\hline 38933 & Chlorpyrifos \\
\hline 61636 & Chlorpyrifos, oxygen analog \\
\hline 79846 & cis-Propiconazole \\
\hline 04031 & Cycloate \\
\hline 61585 & Cyfluthrin \\
\hline 61586 & Cypermethrin \\
\hline 62169 & Desulfinylfipronil amide \\
\hline 39572 & Diazinon \\
\hline 38775 & Dichlorvos \\
\hline 38454 & Dicrotophos \\
\hline 39381 & Dieldrin \\
\hline 82662 & Dimethoate \\
\hline 82677 & Disulfoton \\
\hline 61640 & Disulfoton sulfone \\
\hline 61590 & Endosulfan sulfate \\
\hline 82668 & EPTC \\
\hline 82346 & Ethion \\
\hline 61644 & Ethion monoxon \\
\hline 82672 & Ethoprophos \\
\hline 61591 & Fenamiphos \\
\hline 61645 & Fenamiphos sulfone \\
\hline 61646 & Fenamiphos sulfoxide \\
\hline 62166 & Fipronil \\
\hline
\end{tabular}


Table 1.1. Pesticide compounds not detected in the Cedar River alluvial aquifer, Devonian aquifer, and the Cedar River near Cedar Rapids, Linn County, lowa, 1990-2019.—Continued

[Search in the U.S. Geological Survey (USGS) National Water Information System database (https://doi.org/ $10.5066 / \mathrm{F} 7 \mathrm{P} 55 \mathrm{KJN}$ ) using the parameter code to see full parameter names, descriptions, and other data in the database. EPTC, S-ethyl dipropylthiocarbamate]

\begin{tabular}{|c|c|}
\hline USGS parameter code & Pesticide compound \\
\hline 62168 & Fipronil sulfone \\
\hline 04095 & Fonofos \\
\hline 61593 & Iprodione \\
\hline 61594 & Isofenphos \\
\hline 61595 & $\lambda$-Cyhalothrin \\
\hline 61652 & Malaoxon \\
\hline 39532 & Malathion \\
\hline 61596 & Metalaxyl \\
\hline 61598 & Methidathion \\
\hline 82667 & Methyl parathion \\
\hline 82671 & Molinate \\
\hline 61599 & Myclobutanil \\
\hline 61600 & Oxyfluorfen \\
\hline 61664 & Paraoxon-methyl \\
\hline 82664 & Phorate \\
\hline 61666 & Phorate oxon \\
\hline 61601 & Phosmet \\
\hline 61668 & Phosmet oxon \\
\hline 82679 & Propanil \\
\hline 82685 & Propargite \\
\hline 82676 & Propyzamide \\
\hline 82670 & Tebuthiuron \\
\hline 82675 & Terbufos \\
\hline 61674 & Terbufos oxygen analog sulfone \\
\hline 82681 & Thiobencarb \\
\hline 61610 & Tribufos \\
\hline 04034 & Vernolate \\
\hline
\end{tabular}



For more information about this publication, contact: Director, USGS Central Midwest Water Science Center 400 South Clinton Street, Suite 269

lowa City, IA 52240

319-337-4191

For additional information, visit: https://www.usgs.gov/ centers/cm-water

Publishing support provided by the

Rolla Publishing Service Center 
